\title{
FAULT-FREE INTEGRITY ANALYSIS OF MEGA-CONSTELLATION-AUGMENTED GNSS
}

\author{
by \\ Danielle Racelis
}

Copyright (C) Danielle Racelis 2019

\author{
A Thesis Submitted to the Faculty of the \\ DEPARTMENT OF AEROSPACE \\ AND MECHANICAL ENGINEERING \\ In Partial Fulfillment of the Requirements \\ For the Degree of \\ MASTER OF SCIENCE \\ In the Graduate College \\ THE UNIVERSITY OF ARIZONA
}




\section{THE UNIVERSITY OF ARIZONA \\ GRADUATE COLLEGE}

As members of the Master's Committee, we certify that we have read the thesis prepared by Danielle Racelis, titled Fault-Free Integrity Analysis of Mega-Constellation-Augmented GNSS and recommend that it be accepted as fulfilling the thesis requirement for the Master's Degree.

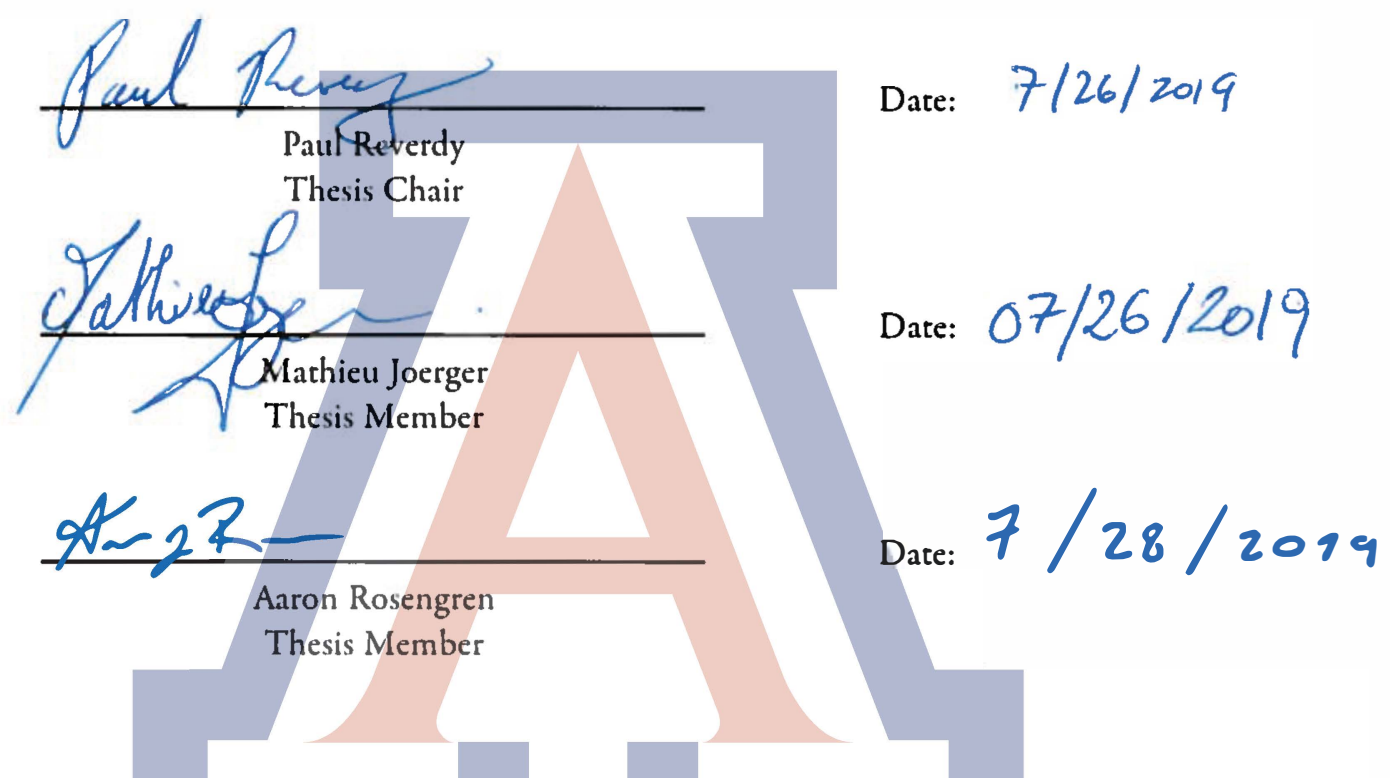

Final approval and acceptance of this thesis is contingent upon the candidate's submission of the final copies of the thesis to the Graduate College.

I hereby certify that I have read this thesis prepared under my direction and recommend that it be accepted as fulfilling the Master's requirement.

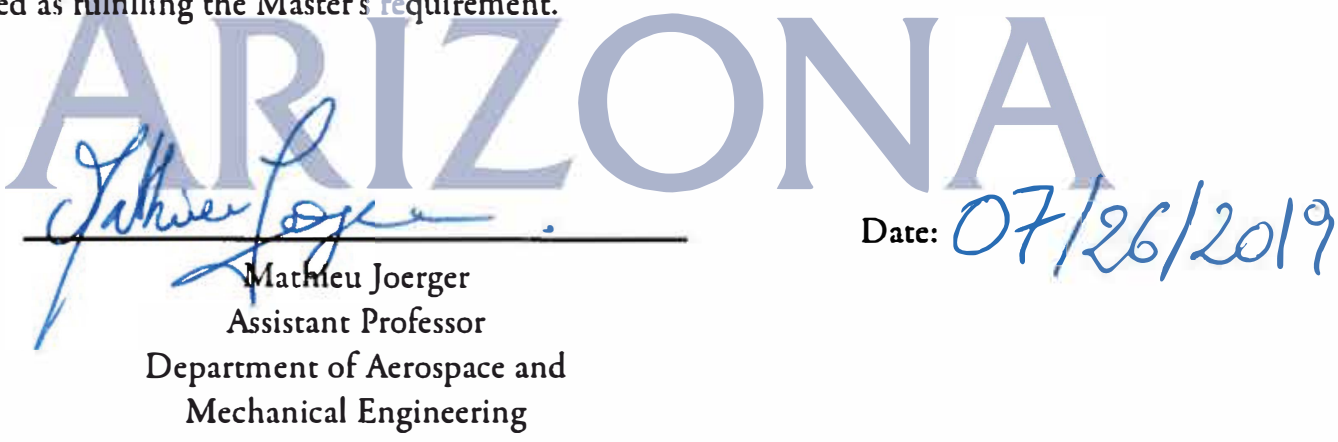




\section{Acknowledgements}

I would like to express my deepest gratitude to my adviser, Professor Mathieu Joerger, for his patient guidance, enthusiastic encouragement, and maddening attention to detail. His advice and assistance have driven me to learn to write quality technical prose, and to become the best researcher I can be. I wish to thank my defense committee, including Professor Paul Reverdy, and Professor Aaron Rosengren, for their willingness to give their time so generously, and their valuable and constructive suggestions.

I would especially like to express my gratitude to Professor Cho Lik Chan who showed me the way through graduate school, and provided advice many times during my studies, to Tanner Campbell for sharing his expertise on all space-related topics, to Jini Kandyil who always kept her door open and had answers to all of my questions, and to my unofficial reviewers Michelle, Alissa, and Quinn, for their very candid and critical insights.

I thank all past and present members of the Navigation Lab: Ali who is extremely knowledgeable in just about everything, and has been my comrade-in-arms in the battlefield that is graduate research, Nick who is my close friend and personal lifestyle guru, Syeed, Salah, Sarah, Patzke, Guillaume, and Lucas, who tolerated being in the same office with me, listening to rants, practice talks, random musings, and offering useful advice on a wide array of topics.

Ako ay lalo na nagpapasalamat sa Garcias, na aking pamilya dito sa Estados Unidos, para sa pabubukas ng kanilang tahanan at kanilang mga puso sa akin.

Nais kong ipahayag ang aking pinakamalalim na pasasalamat sa aking ama, dahil sa kanyang walang pasubali na pag-ibig at matibay na paniniwala sa akin, kasama ang napakalawak na pinansiyal na tulong, at sa aking mga kapatid na si Gabbie at Ro na sumusuporta sa akin sa bawat hakbang ng mahigpit na pagsubok na ito kahit na mula sa kabilang panig ng mundo. Mahal na mahal ko kayo.

Utang ko ang aking mga nakamit kay $\mathrm{D}$, na nag-udyok sa akin na matuto at patuloy na matuto. Ikaw ay bahagi ng aking buhay magpakailanman.

Most importantly, I want to thank Jacob, my best friend and the love of my life. It is entirely possible that I would have given up on this adventure had you not been willing to wake me every morning, I would have succumbed to the pressures of graduate life had you not been beside me every step of the way, and I would have never found faith in myself had you not been as important a part of my life, and of my consciousness, as you are.

This thesis would not be possible without the encouragement and support of numerous entities that I couldn't possibly come up with a full list. You know who you are. Thanks to all. Maraming maraming salamat. 
Dedicated with love to Elizabeth 
$\ldots . .-. . /-\ldots . . / .-. .-.-. . . . / / . . / . . .-\ldots-\ldots . . .-$ 


\section{Contents}

$\begin{array}{ll}\text { List of Figures } & 8\end{array}$

Abstract

1 Introduction $\quad 10$

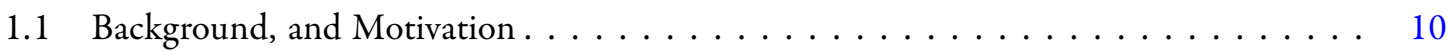

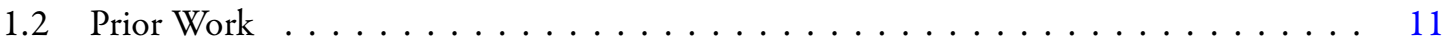

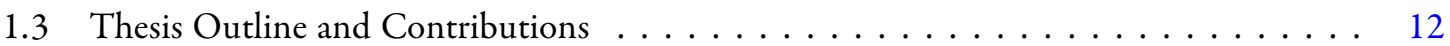

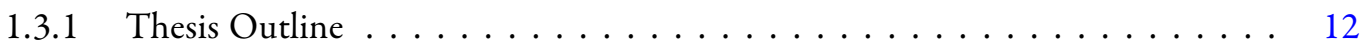

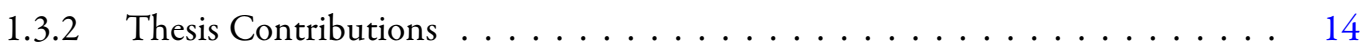

2 GNSS Fundamentals 16

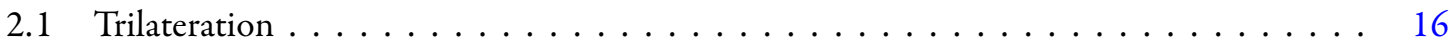

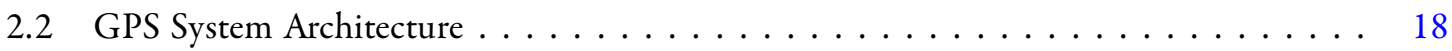

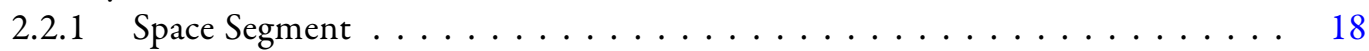

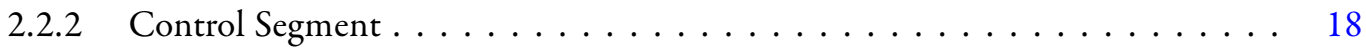

2.2 .3 User Segment . . . . . . . . . . . . . . . . . . . . . . . . . . . . . 19

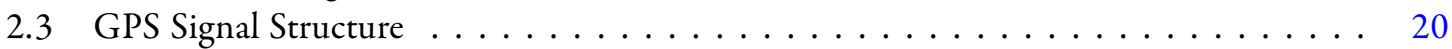

2.4 GPS Measurement Models . . . . . . . . . . . . . . . . . . . 21

2.4 .1 Code Phase Measurements . . . . . . . . . . . . . . . . 21

2.4 .2 Carrier Phase Measurements . . . . . . . . . . . . . . . . 21

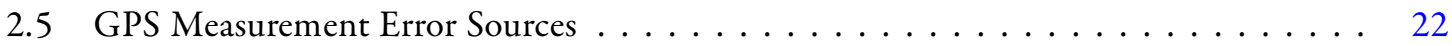

2.5.1 Control Segment Errors: Orbit Ephemeris and Clock Uncertainty . . . . . . . . 22

2.5.2 Signal Propagation Errors: Ionospheric and Tropospheric Delays . . . . . . . . 23

2.5.3 Measurement Tracking Errors: Receiver Noise and Multipath . . . . . . . . . 25

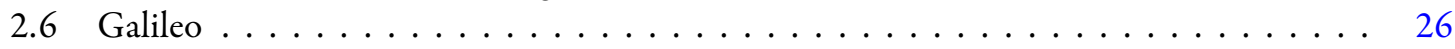

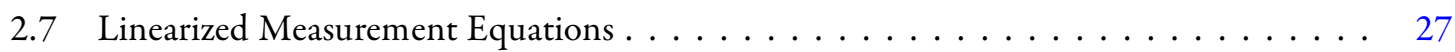

2.8 High Accuracy Positioning Using Carrier Phase . . . . . . . . . . . . . . 28

2.8.1 Relative Positioning Using Carrier Phase: An Illustrative Example . . . . . . . . 28

2.8.2 Point Positioning Using Carrier Phase Measurements from LEO Satellites . . . 30

2.9 Navigation Performance Metrics $\ldots \ldots \ldots \ldots \ldots \ldots \ldots \ldots \ldots \ldots \ldots$ 
3 GNSS-Mega-Constellation System Overview, and Measurement Error Models 32

3.1 Envisioned GNSS-Mega-Constellation System Overview . . . . . . . . . . . . . . 33

3.1 .1 Envisioned Space Segment . . . . . . . . . . . . . . . . . . . . . 34

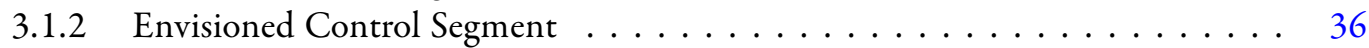

3.1 .3 Envisioned User Segment. . . . . . . . . . . . . . . . . . . . 37

3.2 Nominal Measurement Error Models . . . . . . . . . . . . . . . . . . . . . . 37

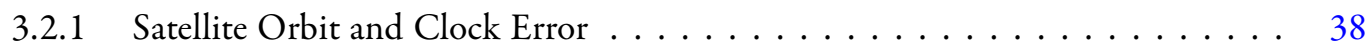

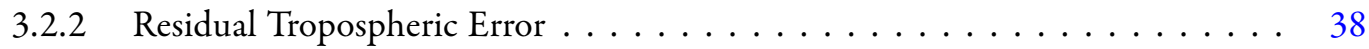

3.2 .3 Multipath Error . . . . . . . . . . . . . . . . . . . . 39

3.2.4 Receiver Noise Error . . . . . . . . . . . . . . . . . . . . . . . . . . . . . 41

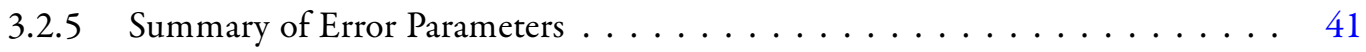

3.3 Measurement Error Modelling Using TLE Data . . . . . . . . . . . . 42

3.3.1 TLE Error Data . . . . . . . . . . . . . . . . . . 43

3.3.2 Gaussian Overbounding Model of TLE Errors for MEO GPS Satellites . . . . 46

3.3.3 Gaussian Overbounding Model of TLE Errors for GEO WAAS Satellites . . . 48

4 GNSS-Mega-Constellation Positioning Algorithm 49

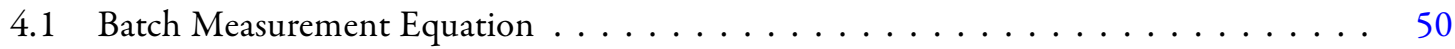

4.2 Batch Measurement Error Covariance Matrix Accounting for Measurement Error Time

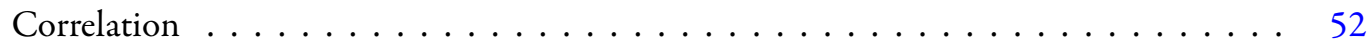

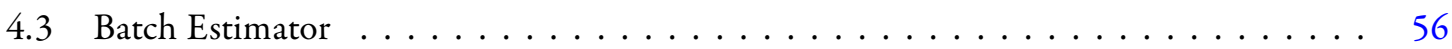

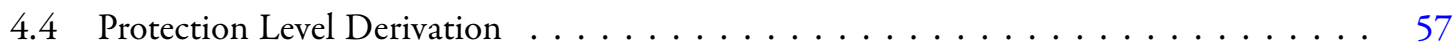

5 Performance Analysis $\quad 59$

5.1 Covariance Analysis Implementation . . . . . . . . . . . . . . . . . . . . 59

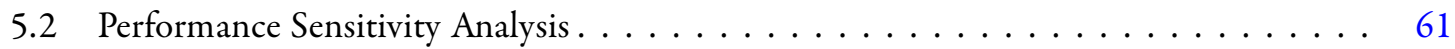

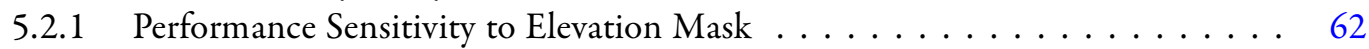

5.2 .2 Performance Sensitivity to Batch Period . . . . . . . . . . . . . . . 66

5.2.3 Performance Sensitivity to Measurement Sampling Rate . . . . . . . . . . . . . . . 68

5.2.4 Performance Sensitivity to Satellite Orbit and Clock Error . . . . . . . . . . . 70

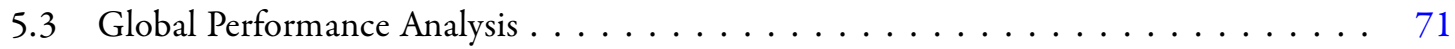

5.3.1 Fault-Free Availability and Coverage Analysis . . . . . . . . . . . . . 71

5.3.2 Fault-Free Availability Analysis for Varying HAL . . . . . . . . . . . . 75

6 Conclusions 77

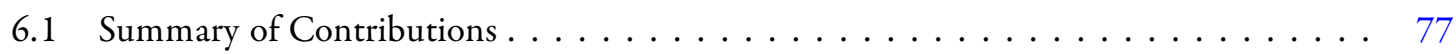

6.2 Recommended Topics for Future Work . . . . . . . . . . . . . . . . . 79

$\begin{array}{ll}\text { Bibliography } & 81\end{array}$ 


\section{List of Figures}

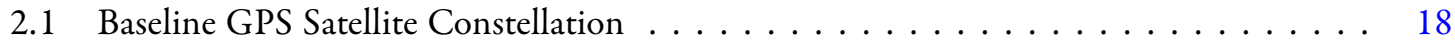

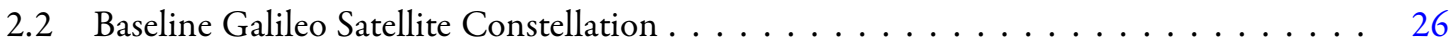

2.3 Simplified Relative Positioning Example . . . . . . . . . . . . . . . . . . . . . 29

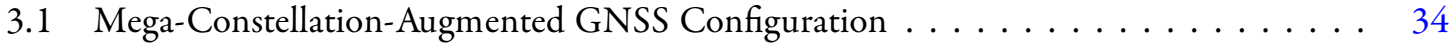

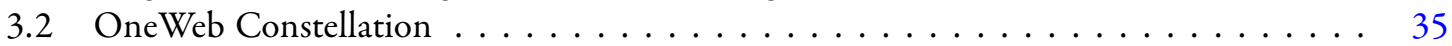

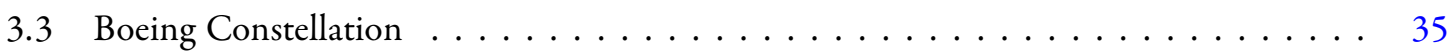

3.4 Mega-Constellation-Augmented GNSS Configurations $\ldots \ldots \ldots \ldots \ldots \ldots$

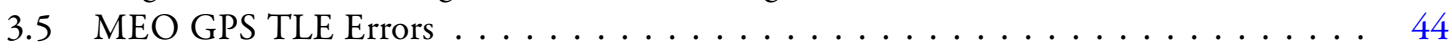

3.6 MEO GPS and GEO WAAS TLE Error Comparison $\ldots \ldots \ldots \ldots \ldots \ldots \ldots \ldots$

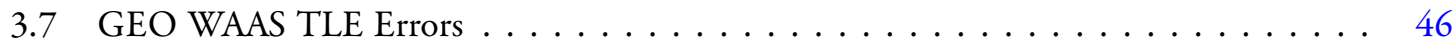

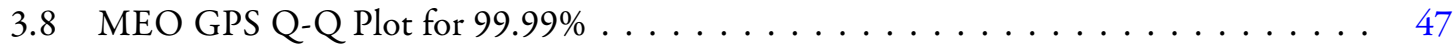

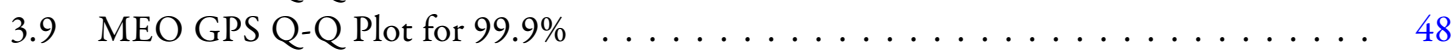

3.10 GEO WAAS Q-Q Plot for $99.9 \% \ldots \ldots \ldots \ldots \ldots \ldots \ldots \ldots$

4.1 Horizontal Protection Level and Alert Limit . . . . . . . . . . . . . . . . . 57

5.1 Performance and AzEl Plots for OneWeb-augmented GNSS Using a 40 Elevation Mask 60

5.2 Obstructed Sky Above a Car-Mounted Antenna . . . . . . . . . . . . . . . . 62

5.3 Elevation Sensitivity Performance Using GNSS Only . . . . . . . . . . . . . . . 63

5.4 Elevation Sensitivity Performance Using GNSS-OneWeb . . . . . . . . . . . . . . 64

5.5 Elevation Sensitivity Performance Using GNSS-Boeing . . . . . . . . . . . . . . . . 65

5.6 Batch Period Sensitivity Analysis Using a $40^{\circ}$ Elevation Mask . . . . . . . . . . . . 67

5.7 Measurement Sampling Rate Sensitivity Analysis Using a $40^{\circ}$ Elevation Mask . . . . . 69

5.8 Ephemeris Error Sensitivity Analysis Using a $40^{\circ}$ Elevation Mask . . . . . . . . . . 70

5.9 Fault-Free Availability Maps for $1.5 \mathrm{~m}$ HAL using GNSS only . . . . . . . . . . . . 72

5.10 Fault-Free Availability Maps for 1.5m HAL using GNSS-OneWeb . . . . . . . . . . . . 73

5.11 Fault-Free Availability Maps for $1.5 \mathrm{~m}$ HAL using GNSS-Boeing . . . . . . . . . . . . 74

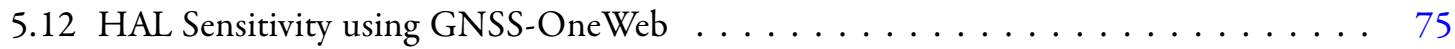

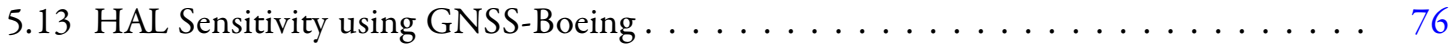




\begin{abstract}
The ultimate in Global Navigation Satellite System (GNSS) performance is obtained using carrier phase measurements. Receiver tracking error for the carrier is lower than that for the code by two to three orders of magnitude, but achieving high accuracy carrier positioning requires that the unknown constant cycle ambiguities be determined. A costless yet efficient solution to this problem is to exploit the bias observability provided by satellite motion. Unfortunately, the large amount of time required for GNSS spacecraft to achieve significant changes in line of sight hinders its use in most real-time applications. In contrast, angular variations from low-Earth orbiting (LEO) satellites quickly become substantial. Therefore, the combination of LEO mega-constellations and GNSS observations makes quick and unambiguous carrier phase positioning possible.

In this thesis, we evaluate the potential of mega-constellation-augmented GNSS (GNSS$\mathrm{MC})$ to provide fault-free high-integrity positioning in both open-sky and urban areas. We derive a method to integrate dual-frequency carrier-phase ranging measurements from GNSS at medium Earth orbit (MEO), and mega-constellations at LEO to achieve global carrier-phase positioning. From the perspective of users on earth, LEO satellites are moving much faster than GNSS at MEO. The large angular variations generated by these fastmoving LEO satellites are exploited for rapid estimation of cycle ambiguities. The addition of mega-constellations to GNSS also improves the spatial diversity of ranging sources which enables improved navigation performance in areas where visible GNSS satellites are too few to obtain a position fix, such as in dense cities and urban canyons. This research helps identify sensitive ground, space, and user segment components that are key to leveraging future communication mega-constellations in safety-critical land navigation applications.
\end{abstract}




\section{Chapter 1}

\section{Introduction}

\subsection{Background, and Motivation}

The success of the Global Positioning System (GPS) has long surpassed the original intentions set forth by its designers when it was launched in the early 1970s. Other Global Navigation Satellite Systems (GNSS) of which GPS was the forerunner, have since been developed by the Soviet Union (GLONASS), the European Union (Galileo), and China (Beidou). In addition, different satellite-based and land-based augmentation systems have been deployed to extend the scope of application of GNSS to safety-critical operations.

The accuracy, integrity, continuity, and availability of GNSS-derived position estimates depend on two key factors: (1) the number of satellites in view and their distribution in the sky, and (2) the characteristics of range measurement errors. In this context, GPS is limited because (1) it was designed for open sky scenarios where only users who have a clear view of the sky will see the minimum required number of satellites, and (2) it was designed to be used with code phase ranging measurements, which can be measured with accuracy no better than meter-level.

Carrier phase positioning is a precise positioning process that uses both code and carrier phase measure- 
ments from GNSS to achieve centimeter-level positioning errors. The carrier phase of a GNSS signal can be tracked to a precision of 0.05 cycles $(1 \mathrm{~cm})$, but these signals were not designed to be used as ranging measurements, and have inherent cycle ambiguities. The carrier phase cycle ambiguities can be estimated by observing large angular variations in the line-of-sight vectors between the user and visible satellites. The large amounts of time needed for GNSS satellites to achieve large angular variations results in long initialization times, and precludes its use in real time applications.

Private companies such as SpaceX, OneWeb, and Boeing are planning to launch mega-constellations composed of hundreds, if not thousands of satellites at low-Earth orbit (LEO) with the intention of providing internet worldwide. If the data's carrier signal can be tracked, then the additional ranging sources provided by these mega-constellations presents opportunities for improved navigation performance in areas where GNSS satellites are partially obstructed. In addition, from the perspective of users near the surface of the Earth, mega-constellation satellites at LEO move much faster than GNSS satellites at medium-Earth Orbit (MEO), and angular variations quickly become substantial for LEO satellites. Therefore, combining measurements from GNSS satellites and LEO mega-constellations makes real-time centimeter-level carrier phase positioning possible.

\subsection{Prior Work}

The use of LEO satellites for improved navigation performance is not completely unexplored. The underlying concept of using spacecraft motion to resolve cycle ambiguities is equivalent to the principle of Doppler positioning used in Transit, the first operational satellite radio-navigation system, whose constellation was also comprised of LEO satellites. Using Transit, the position of stationary receivers could be determined with better than 70 meters of accuracy [1]. The most compelling proof of concept for carrier phase positioning without the stationary user restriction was realized in the late 1990's by Rabinowitz et al [2], with the development of a receiver capable of tracking carrier-phase measurements from GPS and from GlobalStar (another LEO telecommunications constellation). Using geometry variations from fast moving GlobalStar satellites, precise cycle ambiguity resolution and positioning 
with respect to a nearby reference station was achieved.

In [3], the augmentation of GPS with LEO Iridium satellite signals provided signal redundancy and enabled fault-detection using carrier phase Advanced Receiver Autonomous Integrity Monitoring (ARAIM). Another implementation can be found in [4] where LEO satellite signals were used to limit the drift of inertial sensors in GPS-denied areas. More recent work in [5] examined the major components that could allow soon to be deployed mega-constellations to act as navigation satellites if they hosted a dedicated payload.

\subsection{Thesis Outline and Contributions}

This research focuses on safety-critical navigation applications where integrity is of primary concern. Integrity risk is the probability of undetected faults causing unacceptably large positioning errors. Can mega-constellations, when combined with GNSS, contribute to achieving fault-free safety-critical integrity risk requirements? Can they do so in urban areas where low-elevation satellite signals are blocked?

In response, in this work, we quantify the fault-free integrity performance of mega-constellation-augmented GNSS (GNSS-MC) in both open sky and urban environments. This preliminary analysis aims at identifying the key tradeoffs and the most sensitive parameters that would allow such a multi-constellation system to achieve fault-free integrity requirements.

\subsubsection{Thesis Outline}

The second chapter of this thesis provides a background on GNSS fundamentals including trilateration, the space, ground, and user segments of GPS, measurement models and error sources, and positioning using carrier phase. This chapter should provide the necessary groundwork for the discussions found in subsequent chapters.

The first section of Chapter 3 describes a notional system architecture for the combined constellation. 
Since mega-constellations are primarily intended for communication, assumptions are made regarding the space, ground, and user segments that would allow these LEO satellites to be used for navigation. We consider a candidate architecture where LEO orbit and clock determination is done through a dedicated network of ground stations. The second section deals with the measurement error models for the combined system, based on the notional architecture outlined in the first section. Signals from fast-moving LEO satellites cross wide sections of the atmosphere within a few minutes, which makes modeling of ranging errors due to the ionosphere and troposphere extremely challenging. Additionally, addressing ionospheric anomalies in safety-critical applications is exacting $[6,7]$. In this work, we assume dual-frequency LEO carrier measurements, which are used to eliminate the impact of ionospheric errors. The remaining errors including orbit and clock ephemeris errors, tropospheric delay, multipath errors, and receiver noise are modeled conservatively over time as recommended in [8]. The final section of Chapter 3 describes and implements a methodology for high-integrity error modeling of example data in the form of Two-Line Elements (TLEs). The discussion of TLE error modeling illustrates an important building block of integrity analysis called overbounding theory, which allows us to determine probabilistic bounds on empirical error distributions.

The fourth chapter describes the batch measurement equation, weighted least squares algorithm, and the protection level (PL) equation. The PL defines a probabilistic bound on positioning error. The PL is set so that the risk of the positioning error exceeding the PL is smaller than a predefined integrity risk requirement. Ionosphere-free GNSS code and carrier measurements, and mega-constellation carrier measurements are processed in a batch to simultaneously estimate three-dimensional position, receiver clock biases (one per constellation when constellations are not synchronized), and cycle ambiguities.

In the fifth chapter, the horizontal positioning performance of the combined constellation is evaluated. We first analyze the positioning error sensitivity at an example location to elevation mask, batch period, measurement sampling rate, and ephemeris error standard deviation for three configurations: GNSS only, GNSS-OneWeb, and GNSS-Boeing. This local sensitivity analysis quantifies the improvement 
brought about by GNSS-MC over GNSS alone. Finally, we carry out a worldwide fault-free availability analysis for the combined constellation system when used in open sky versus urban canyon environments, and put into perspective the capability of mega-constellations to be used for safe navigation.

\subsubsection{Thesis Contributions}

Derivation of Nominal Measurement Error Models for LEO Mega-Constellations. High-integrity GNSS-based navigation algorithms only require instantaneous or snapshot measurement error models, which are insufficient for time-sequential processing. Also, for a user near the surface of the Earth, LEO satellites cross the sky faster than MEO GNSS spacecraft, causing signals to traverse large portions of the atmosphere. In this work, we review existing error models and design a notional ground, space, and user segment architecture for both mega-constellation-augmented GNSS (GNSS-MC). We then derive new carrier phase measurement error models over time for satellite clock and orbit ephemeris errors, atmospheric propagation delays, multipath and receiver noise. These models are key to the development and integrity analysis of GNSS-MC time-sequential algorithms in land-vehicle application.

Probabilistic Error Modeling Using Two-Line Elements (TLEs). Error models must be validated using large amounts of experimental data. The error models need to be conservative enough to be safe, but not overly conservative or the system would too often indicate that it is not trustworthy. Such validation was carried out before for GNSS orbit ephemeris data, but has never been performed for TLEs, which is the format of the largest publicly available catalogue of space objects. In this research, we processed and analyzed 20 years of TLE data for a set of MEO and geostationary Earth-orbiting (GEO) satellites. Based on this data, we derive high-integrity TLE error models using overbounding theory to determine probabilistic bounds on the radial, along-track, and cross-track satellite positioning errors. The approach illustrates the main steps of orbit error modeling for future mega-constellation satellites, and is relevant to other applications including space situational awareness [9]. 
Horizontal Positioning Error Sensitivity Analysis and Worldwide Fault-Free Availability Analysis. In this research, we evaluate the horizontal positioning performance of GNSS-MC against varying elevation masks, batch periods, measurement sampling rates, and satellite ephemeris errors. This sensitivity analysis was first implemented for a single location to quantify the improvements brought about by augmenting GNSS with mega-constellations as compared to using GNSS alone. We then perform a worldwide fault-free availability analysis by assessing the fault-free integrity risk over time for a grid of locations on Earth. This performance evaluation helps identify the scope of applications potentially enabled by GNSS-MC. 


\section{Chapter 2}

\section{GNSS Fundamentals}

This chapter will primarily cover the fundamentals of GNSS navigation as it pertains to GPS, which is the United States' GNSS, and will provide a shorter section on Galileo, the European GNSS which shares many similarities with GPS. The objective is to provide a succinct account of the topics relevant to this thesis. The discussion included herein follows closely Misra and Enge's text [10].

This chapter will cover the method of radionavigation employed by GNSS called trilateration, the space, ground, and user segments of GNSS that make trilateration possible, and the challenges involved with the non-ideal performance of GNSS (i.e. measurement error sources). We give a background on precise positioning using carrier phase and provide an example that illustrates the role of geometric diversity in carrier phase navigation. Lastly, we cover the required navigation performance metrics, and their role in the performance analysis.

\subsection{Trilateration}

The basic idea behind trilateration is that a user's unknown three-dimensional position can be determined by measuring its distance from three known locations. By this principle, a user on Earth can determine their position by measuring its distance from satellites at known positions. Satellites at known locations transmit electromagnetic signals that travel at a known speed. A user on the ground can receive 
this signal, and by measuring its transit time, can determine its distance to the satellite. The method of radionavigation used by GPS is based on this principle and is referred to as a time-of-arrival system [10]. In principle, if a user can measure its distance to three different satellites at known positions perfectly, then the user's three-dimensional position can be determined unambiguously.

The distance between GPS receiver and satellite is measured in terms of transit time of the signal from the satellite to the receiver. Precise measurement of transit time requires that clocks on the receiving and transmitting side be synchronized. Clocks on GPS satellites can be steered to remain synchronized, but the receiver clock will always have an offset. This error is called receiver clock bias. Fortunately, the bias in the receiver clock at the instant of measurement affects the observed transit time on all the satellites equally. This means all the measured ranges will be too long, or too short by the same amount. These biased range measurements are referred to as pseudoranges. The receiver clock bias then becomes an additional unknown. A minimum of four satellites is now needed to determine three coordinates of spatial position and time, and receiver clocks can keep using inexpensive quartz oscillators. The idealized pseudorange measurement equation is given below.

$$
\begin{aligned}
&{ }^{i} \rho=\sqrt{\left({ }^{i} x-x\right)^{2}+\left({ }^{i} y-y\right)^{2}+\left({ }^{i} z-z\right)^{2}}+\tau_{u} \quad i=1,2, \ldots, n \\
&{ }^{i} \rho: \text { Pseudoranges (measurements) } \\
&\left({ }^{i} x,{ }^{i} y,{ }^{i} z\right): \text { Satellite positions (known) } \\
&(x, y, z): \text { User position (unknown) } \\
& \tau_{u}: \text { Receiver clock bias (unknown) }
\end{aligned}
$$

In order to solve for the four unknowns in Eq. 2.1, a user needs at least four measurements, which means that at least four satellites must be in view of the receiver. Moreover, our estimates of these four unknowns, will only be as good as our knowledge of the satellite positions and clocks. 


\subsection{GPS System Architecture}

GPS is comprised of three segments: the space segment, the control segment, and the user segment. The space segment is composed of the satellites, the control segment encompasses all infrastructure that monitors and maintains satellite operations, and the user segment includes all receivers and services that use GPS.

\subsubsection{Space Segment}

The baseline GPS constellation is comprised of 24 satellites in near circular orbits that have a radius of $26,560 \mathrm{~km}$, an orbital period of approximately twelve hours, and quasi-stationary ground tracks. The satellites are distributed among six orbital planes with an inclination of $55^{\circ}$. There are four primary satellite slots, and one spare slot distributed unevenly in each orbital plane, which allow the constellation to hold up to thirty satellites on orbit. Figure 2.1 shows the nominal 24-GPS satellite constellation used in the simulations.

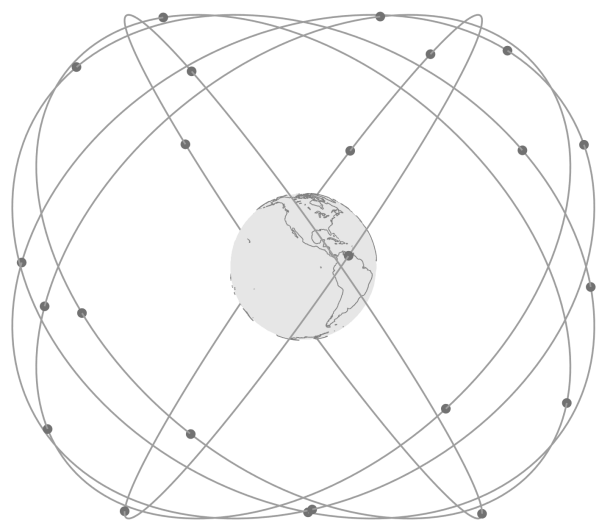

Figure 2.1: Baseline GPS Satellite Constellation. There are 24 satellites in six orbital planes inclined at $55^{\circ}$, in near circular orbits with radius 26,560 $\mathrm{km}(20,200 \mathrm{~km}$ altitude), with orbital period of $11 \mathrm{~h} 58 \mathrm{~m}$, and ground tracks that repeat every sidereal day.

The baseline constellation was designed to ensure that there are at least four satellites in view for all users with an unobstructed view of the sky. The satellites broadcast ranging signals and navigation data that allow users to estimate their position. The computational work needed to determine the satellite orbits, and therefore their positions, is done by the ground control segment.

\subsubsection{Control Segment}

Satellites at medium-Earth orbit (MEO) are susceptible to forces that skew the orbit from a perfect ellipse. In order to maintain accurate knowledge of GPS orbits, the satellites are monitored by a dedicated global network of ground stations that comprise the control segment of GPS. The satellite ephemeris 
and clock parameters are predicted 24 hours in advance by the control segment using models derived from actual observations of the satellites. The predicted satellite ephemeris and time synchronization information is included in the navigation message uploaded to the satellites once every two hours [11] (valid for overlapping four hours). The navigation message is then broadcast by each satellite in a 50 bits-per-second data stream to the users. The control segment is in charge of monitoring satellite orbits, monitoring and maintaining satellite health, maintaining GPS time, predicting satellite ephemerides and clock parameters, updating satellite navigation messages (containing the satellite ephemeris, clock parameters, and other information needed for user positioning), and commanding station keeping maneuvers [10].

\section{GPS Time}

GPS uses and maintains its own time reference called GPS Time (GPST), which is different from the international civil standard called UTC. To ensure that all satellite clocks are synchronized, the control segment monitors the time offset, drift, and drift rate of each satellite clock relative to GPS Time, and the parameters of the model for this bias is broadcast by each satellite in the navigation message [10].

\subsubsection{User Segment}

The space segment and control segment providing GPS ranging signals and navigation messages, are distinct from the user segment responsible for the acquisition and processing of GPS signals. For our purposes, a receiver is both a radio receiver and digital computer capable of "listening" to the transmitted GPS signals, processing them to determine the range to each satellite as well as the positions of the satellites, and calculating the user position in useful coordinates [12]. How well GPS signals are acquired and processed is highly variable between receivers. The widespread use of GPS is due in large part to commercially-led advancements in receiver technology that allowed GPS receivers to be placed in smartphones and used for everyday navigation. An in-depth discussion of receiver technology will not be provided here. However, it is worth going over the basic structure of GPS signals to better understand the concepts behind carrier phase positioning. 


\subsection{GPS Signal Structure}

Modern GPS satellites can transmit continuously on radio frequencies in the L-band referred to as Link 1 (L1), Link 2 (L2), and Link 5 (L5). These are the four signals available for civilian use: L1 C/A, L2C, L5, and L1C. The legacy signal L1 C/A is broadcast by all satellites. The other three modernized signals are not broadcast by all satellites [13]. L1 and L5 are protected navigation bands. This work looks at future performance where every GPS satellite will broadcast both L1 C/A and L1C signals on L1, and on L5. L1 and L5 have center frequencies $f_{\mathrm{L} 1}=1575.42 \mathrm{MHz}$, and $f_{\mathrm{L} 5}=1176.45 \mathrm{MHz}$.

Each signal has three components: carrier, ranging code, and navigation data. The carrier is a sinusoidal signal with frequency $f_{\mathrm{L} 1}$, or $f_{\mathrm{L} 5}$. The ranging code refers to a family of binary codes called pseudo-random noise (PRN) sequences or PRN codes unique to each satellite. The PRN codes are designed to allow all satellites to broadcast at the same frequency without interfering with each other, following the principle of code division multiple access (CDMA). There are different codes associated with each service i.e. the Standard Positioning Service (SPS) available to civilians and the Precise Positioning Service (PPS) restricted to the U.S. military. This work deals solely with SPS, so we will be using SPS codes that are called coarse/acquisition codes (C/A-codes) (as opposed to PPS codes which are referred to as precision (encypted) codes, or $\mathrm{P}(\mathrm{Y})$-codes) [10]. In this work, we refer to ranging code, PRN code, and C/A-code simply as code. The third component is the navigation data. The navigation data includes the satellite ephemeris, clock bias parameters, and health status, as well as an almanac consisting of reduced-precision ephemeris data on all active satellites in the constellation. The almanac is processed by the receiver and used to get an idea of which satellite signals to expect.

The binary ranging code is combined with the binary navigation data. The resulting composite binary signal is modulated onto the carrier, through a specific modulation scheme called binary phase shift keying. This short discussion of signal structure should be adequate for the purposes of this thesis. An exhaustive discussion of GPS signals is given in the Interface Specification for Standard Positioning Service (SPS) signals document [14] which is public. 


\subsection{GPS Measurement Models}

Recall the two key factors that affect the quality of a GNSS-derived position estimates: visible satellite count and satellite geometry, and range measurement error characteristics. We talked a little bit about the number of visible satellites earlier in this chapter. We address the quality of measurements in this section, and discuss satellite geometry later in section 2.8. GPS provides two types of measurements: code and carrier phase measurements.

\subsubsection{Code Phase Measurements}

Code phase measurements allow the receiver to determine its range to the satellite through the time difference between when the signal was received by the user as determined by the receiver clock, and when the signal was transmitted by the satellite as determined by the satellite clock. Code phase measurements are modeled as

$$
\rho=r+c\left[\delta t_{u}-\delta t^{s}\right]+\mathscr{E}_{\rho}
$$

where

$$
\begin{array}{ll}
r & \text { is the true geometric range between the user and the satellite, } \\
c & \text { is the speed of light in vacuum, } \\
\delta t_{u} & \text { is the user or receiver clock offset from GPS Time, } \\
\delta t^{s} & \text { is the satellite clock offset from GPS Time, and } \\
\mathscr{E}_{\rho} & \text { denotes measurement errors in the code phase. }
\end{array}
$$

Instead of measuring the true range $r$, what is actually measured is a biased and noisy pseudorange $\rho$ [10]. Equation (2.2) has units of distance and makes no reference to satellite ID or carrier frequency (L1 or L5). We take this generic approach to GPS measurements for the time being.

\subsubsection{Carrier Phase Measurements}

The carrier phase of a GPS signal is measured as the phase difference between the carrier phase signal received from the satellite, and a replica of that signal generated by the receiver clock [10]. Carrier phase is measured in cycles or fractions of cycles. Since carrier signals are uniform periodic wave forms, we 
can only measure the fractional phase difference and not the number of whole cycles generated since the instant of transmission. This unknown number of cycles is referred to as integer cycle ambiguity or simply cycle ambiguity. Thus, carrier phase measurements are an indirect and ambiguous measurement of signal transit time. Carrier phase measurements are modeled as

$$
\phi=r+c\left[\delta t_{u}-\delta t^{s}\right]+\lambda N+\mathscr{E}_{\phi}
$$

where

$$
\begin{array}{ll}
\lambda & \text { is the carrier wavelength, } \\
N & \text { is the integer ambiguity, } \\
\mathscr{E}_{\phi} & \text { denotes measurement errors in the carrier phase, }
\end{array}
$$

and as before, $r$ is the true geometric range between the user and the satellite, and $c$ is the speed of light in vacuum. The carrier wavelength is $\approx 19 \mathrm{~cm}$ at $\mathrm{L} 1$, and $\approx 24 \mathrm{~cm}$ at $\mathrm{L} 2$. If the integer cycle ambiguities can be resolved, then we can take advantage of the precision inherent in carrier phase measurements. We will look at techniques for integer ambiguity estimation in section 2.8.

\subsection{GPS Measurement Error Sources}

Code measurements are unambiguous but coarse, and carrier phase measurements can be accurate if the integer ambiguities are resolved. Both measurement types are affected by modeling and measurement errors. The accuracy of position estimates using using both code and carrier depend heavily on accounting for or eliminating the errors in equations (2.2) and (2.3).

\subsubsection{Control Segment Errors: Orbit Ephemeris and Clock Uncertainty}

Each satellite's state (position and velocity), and clock offset (phase bias, frequency bias, and frequency drift rate) are continuously monitored by the control segment. The control segment takes measurements and uses a Kalman filter to determine the satellite states, and clock offsets. The control segment is also responsible for predicting the satellite orbit ephemeris and clock parameters broadcast by GNSS satellites in the navigation message. The pseudorange error due to uncertainties in the ephemeris and 
clock parameters is continuously estimated and tracked by the control segment in real time, and kept within the root-sum-square (rss) range of $1.5 \mathrm{~m}$ each [10].

\subsubsection{Signal Propagation Errors: Ionospheric and Tropospheric Delays}

When a GPS signal travels from the satellite to the user, it travels about $95 \%$ of the way at a constant speed $c=299,792,458 \mathrm{~m} / \mathrm{s}$, and then passes through the atmosphere for the remainder of its journey. First it passes through the region of space around the Earth from $1000 \mathrm{~km}$ to $40 \mathrm{~km}$ altitude made up of charged particles called the ionosphere. As the signal travels through an altitude of $40 \mathrm{~km}$ up to the Earth's surface, it passes through an electrically neutral gaseous layer called the troposphere. These two layers of the atmosphere change the velocity of propagation of radio waves through a phenomenon called refraction. The change in direction is negligible, but the change in speed shortens or lengthens signal transit time, and can cause the pseudorange measurement to be off by several meters or more.

The refractive index of a medium is defined as the ratio of the speed of light in vacuum $(c)$ to the speed of propagation in the medium. When the medium's refractive index depends on the frequency of the signal, it is referred to as a dispersive medium. The ionosphere is dispersive to radio waves, while the troposphere is not. The composition of the ionosphere and troposphere is not uniform and so the refractive index along the signal path changes. We discuss how we model this in the next subsections.

\subsubsection{Ionospheric Errors}

The refraction of GPS signals through the ionosphere depends on the amount of free electrons in the signal path, called total electron content or TEC. TEC is the number of electrons in a tube of $1 \mathrm{~m}^{2}$ cross section extending from the satellite to the receiver [10]. Signals that are received from zenith have the shortest path length, and therefore encounter the least TEC. Signals that are received at a lower elevation angle have to traverse longer paths through the ionosphere, and in general, TEC increases as the elevation angle decreases. 
The ionization of gas in the ionosphere is due primarily to the sun's radiation. The influence of the ionosphere considerably varies between day and night, between seasons, and between phases of the 11 year long solar cycle. The ionosphere is also affected by solar activity, geomagnetic disturbances, and short term disturbances that do not coincide with known cycles, and are hard to predict. Maps of TEC have been developed from a time history of observations. These maps typically provide TEC values at zenith, and use scaling coefficients called obliquity factors to take into account the satellite elevation [15].

As previously mentioned, the ionosphere is dispersive to radio waves. Due to some wonderful EM physics, the code phase is delayed (measured too long), and the carrier phase is advanced (measured too short) by an equal amount. This is known as code-carrier divergence, and is a result of the carrier travelling slower than the modulation through the ionosphere. The ionospheric delay in the code and carrier phase equations (2.2) and (2.3), are opposite in sign and equal in magnitude [10].

\section{Dual-Frequency Measurements}

A combination of measurements at two separated frequencies (e.g. L1 and L5) allows the elimination of ionospheric delay. However, the ionosphere-error free measurements are noisier than measurements at L1 or L5 alone. The ionosphere-error-free noise inflation factor appears as the multiplier $c_{\mathrm{IF}}$ in the measurement error models in Chapter 3.

\subsubsection{Tropospheric Errors}

GPS signals travel slower through the troposphere than in vacuum, and the effect is that the measured range to the satellite is too long. Unlike the ionosphere, the troposphere is non-dispersive to radio waves, and GPS measurements for code and carrier, L1 and L2, experience a common delay. To mitigate the tropospheric errors we need to use models. The troposphere is composed of dry gases and water vapor which affect the propagation of GPS signals differently, and are modeled separately. The tropospheric delay is dependent on the refractive index of the air mass along its path, which in turn is dependent on the densities of the dry air composition and water vapor, which are functions of the pressures of the dry air 
and water vapor, and the temperature [10]. Many tropospheric models are available with their associated mapping functions. Tropospheric models define dry and wet delays for the zenith direction depending on latitude, altitude, and season. The mapping functions use obliquity factors to take into account satellite elevation (i.e. signals from low elevation angles pass through more air mass than signals from zenith). The obliquity factor increases as the satellite gets lower in the sky [15]. This elevation-dependent tropospheric mapping appears in Eq. (3.8) of Chapter 3, where we discuss the measurement error models for mega-constellation-augmented GNSS (GNSS-MC).

\subsubsection{Measurement Tracking Errors: Receiver Noise and Multipath}

So far, control segment errors, and signal propagation modeling errors were common to code and carrier phase measurements except for the sign change in the ionospheric delay. The last two error sources namely receiver noise and multipath, have effects on code and carrier phase measurements that differ significantly in magnitude.

\subsubsection{Receiver Noise Error}

Receiver noise is a term used to broadly describe the RF radiation processed by the receiver in frequencies unrelated to the desired frequency band. This includes noise generated internally by the receiver's circuits (e.g. thermal noise and imperfections in the electronics), and noise generated from an external source (e.g. background noise and interference from man made signals) [16]. If receivers were perfect, and there were no interfering signals, the signal received is identical to that which was transmitted. In reality, the ideal case is that the signal received is the sum of GPS signals, and randomly fluctuating noise. The resulting signal is noisy, and there is loss of precision in code and carrier phase measurements in disparate amounts. In addition, receiver noise error depends on signal strength which in turn is a function of satellite elevation angle. Received signal strength decreases as the satellite gets closer to the horizon [10]. 


\subsubsection{Multipath Error}

Multipath error is due to signals reaching the antenna from multiple paths other than the direct line of sight. The resulting received signal is a sum of the GPS signal and multiple reflections that are delayed and typically weaker versions of the direct signal. There is multipath "immunity" built into the signal structure of the code, where reflected signals that are delayed by at least a certain amount are suppressed in the auto-correlation process performed at the receiver. Such delays are still two orders of magnitude larger than the corresponding errors for carrier phase measurements simply because the errors for carrier phase can be no worse than a cycle [10].

\subsection{Galileo}

Galileo is the Global Navigation Satellite System (GNSS) developed and launched by the European Union, that started offering early operational capability at the end of year 2016 [17]. The planned constellation has 30 satellites in MEO, with ten satellites placed in each of the three orbital planes [18]. This work assumes 24 satellites distributed among three orbital planes as shown and described in Figure 2.2. Galileo signals are pervaded by similar error sources as GPS and in a similar fashion, but there are certainly differences.

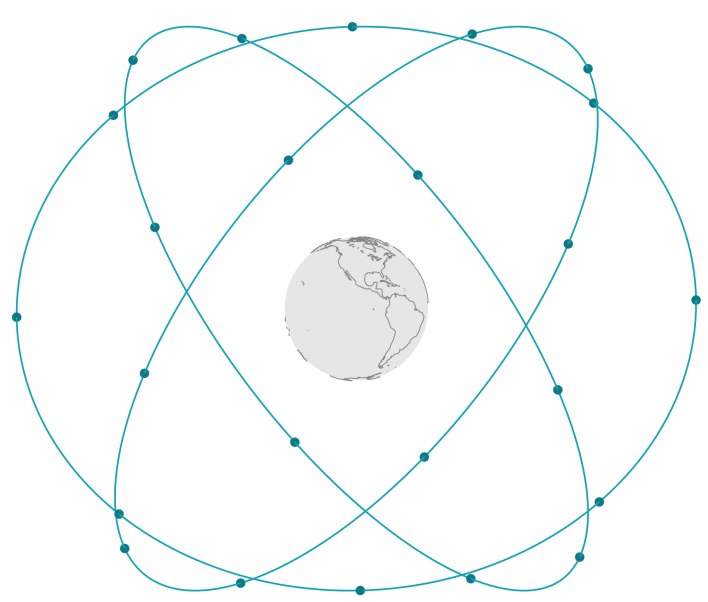

Figure 2.2: Baseline Galileo Satellite Constellation. There are 24 satellites in three orbital planes inclined at $56^{\circ}$, in near circular orbits with radius $29,994 \mathrm{~km}(23,222 \mathrm{~km}$ altitude), with orbital period of $14 \mathrm{~h} 7 \mathrm{~m}$, and ground tracks that repeat every ten days.

The constellation's performance is still improving, and work is being done to develop error models specific to Galileo $[19,20,21]$. In this preliminary work, no distinction has been made between the measurement error models for GPS and Galileo. 


\subsection{Linearized Measurement Equations}

Before linearizing the measurement equations for code and carrier, the satellite clock offset $\delta t^{s}$ is corrected using parameters in the navigation message. The measurement models for code and carrier, Eq. (2.2) and (2.3) are rewritten as

$$
\begin{aligned}
\rho & =r+c \delta t_{u} \quad+\mathscr{E}_{\rho} \\
\phi & =r+c \delta t_{u}+\eta+\mathscr{E}_{\phi}
\end{aligned}
$$

where $\eta=\lambda N$. We now linearize the measurement equation for code using the derivation in [10]. Following the notation we used in equation $(2.1)$, let vector $\mathbf{x}=(x, y, z)$ be the user position at the time of measurement, vector ${ }^{i} \mathbf{x}=\left({ }^{i} x,{ }^{i} y,{ }^{i} z\right)$ be the position of satellite $i$ at the time of signal transmission, and the user clock bias term $c \delta t_{u}$ be replaced by $\tau$. The true range to satellite $i$ is ${ }^{i} r=\left\|{ }^{i} \mathbf{x}-\mathbf{x}\right\|$ and the code measurement equation for satellite $i$ can be rewritten as

$$
{ }^{i} \rho=\left\|{ }^{i} \mathbf{x}-\mathbf{x}\right\|+\tau+{ }^{i} \mathscr{E}_{\rho}
$$

The linearization is about an initial guess $\mathbf{x}_{0}$ and $\tau_{0}$, where $\mathbf{x}=\mathbf{x}_{0}+\delta \mathbf{x}_{0}$ and $\tau=\tau_{0}+\delta \tau_{0}$. Using a Taylor series approximation of a vector norm, we get [10]:

$$
\begin{aligned}
{ }^{i} \delta \rho & ={ }^{i} \rho-{ }^{i} \rho_{0} \\
& =\left\|{ }^{i} \mathbf{x}-\mathbf{x}_{0}-\delta \mathbf{x}\right\|-\left\|^{i} \mathbf{x}-\mathbf{x}_{0}\right\|+\left(\tau-\tau_{0}\right)+{ }^{i} \mathscr{E}_{\rho} \\
& \approx-\frac{{ }^{i} \mathbf{x}-\mathbf{x}_{0}}{\left\|{ }^{i} \mathbf{x}-\mathbf{x}\right\|} \cdot \delta \mathbf{x}+\delta \tau++{ }^{i} \mathscr{E}_{\rho} \\
& =-{ }^{i} \mathrm{e} \cdot \delta \mathbf{x}+\delta \tau+{ }^{i} \mathscr{E}_{\rho}
\end{aligned}
$$

where $-{ }^{i} \mathrm{e}$ is the $3 \times 1$ estimated unit line-of-sight vector directed from the satellite $i$ to the user. Keeping in mind that the linearized equations deal with state deviations, we drop the $\delta$ 's and write the code phase measurement at the time of measurement $k$. The linearized carrier phase measurement equation can be derived the same way and is also written below for completeness.

$$
\begin{aligned}
& { }^{i} \rho_{k}=-{ }^{i} \mathrm{e}_{k}^{\mathrm{T}} \mathbf{x}_{k}+\tau_{k}+{ }^{i} \mathscr{E}_{\rho, k} \\
& { }^{i} \phi_{k}=-{ }^{i} \mathrm{e}_{k}^{\mathrm{T}} \mathbf{x}_{k}+\tau_{k}+\eta+{ }^{i} \mathscr{E}_{\phi, k}
\end{aligned}
$$


We will see these equations again when we discuss the measurement error models and position estimation algorithm in Chapter 3 and 4 respectively.

\subsection{High Accuracy Positioning Using Carrier Phase}

Precise positioning can be achieved with errors at the centimeter level. Coarse and unambiguous code phase measurements with its 300-meter wavelength can only provide positioning accuracy no better than meter-level, while precise but ambiguous carrier phase measurements with its wavelength of $\approx 19 \mathrm{~cm}$ at L1, and $24 \mathrm{~cm}$ at $\mathrm{L} 2$ can potentially provide centimeter-level positioning accuracy, if the integer cycle ambiguities can be resolved. In this section, we look at the fundamental idea behind cycle ambiguity estimation through a simple example.

\subsubsection{Relative Positioning Using Carrier Phase: An Illustrative Example}

We begin this section with an idealized example of precise relative positioning using carrier phase measurements. This simple one-dimensional relative positioning problem should illustrate the rudimentary concepts of integer ambiguity estimation using satellite geometry change, and allude to the complications of precise absolute positioning that motivated this thesis. The ensuing discussion closely follows an example first conceived by Patrick Hwang in [22] and then improved in [10].

Two antennas located at $A$ and $B$ are separated by a baseline $d$, and are tracking the carrier phase of a single satellite. The objective is to determine the baseline distance $d$. The plane wave front of the carrier phase reaches antenna $B$ first. After several full cycles $N$ and a fractional cycle $\Delta_{0}$, the plane wave front reaches antenna $A$. We freeze the image at the instant the wave front reaches $A$, when the satellite is at an angle $\theta_{0}$ as depicted in Figure 2.3. We denote the carrier phase measurements at $A$ and $B$ as $\phi_{A}$ and $\phi_{B}$ respectively. As this time instant $t_{0}$, the difference in carrier phase measurements in units of cycles is

$$
\phi_{A B}\left(t_{0}\right)=\phi_{A}\left(t_{0}\right)-\phi_{B}\left(t_{0}\right)=\Delta_{0}+N
$$

where the phase difference is the sum of a known fractional cycle $\Delta_{0}$ and an unknown number of full 
cycles $N$. If $N$ is known, then the geometric relationship between the two antennas and the satellite gives the baseline distance as

$$
d \cos \left(\theta_{0}\right)=\lambda\left(\Delta_{0}+N\right)
$$
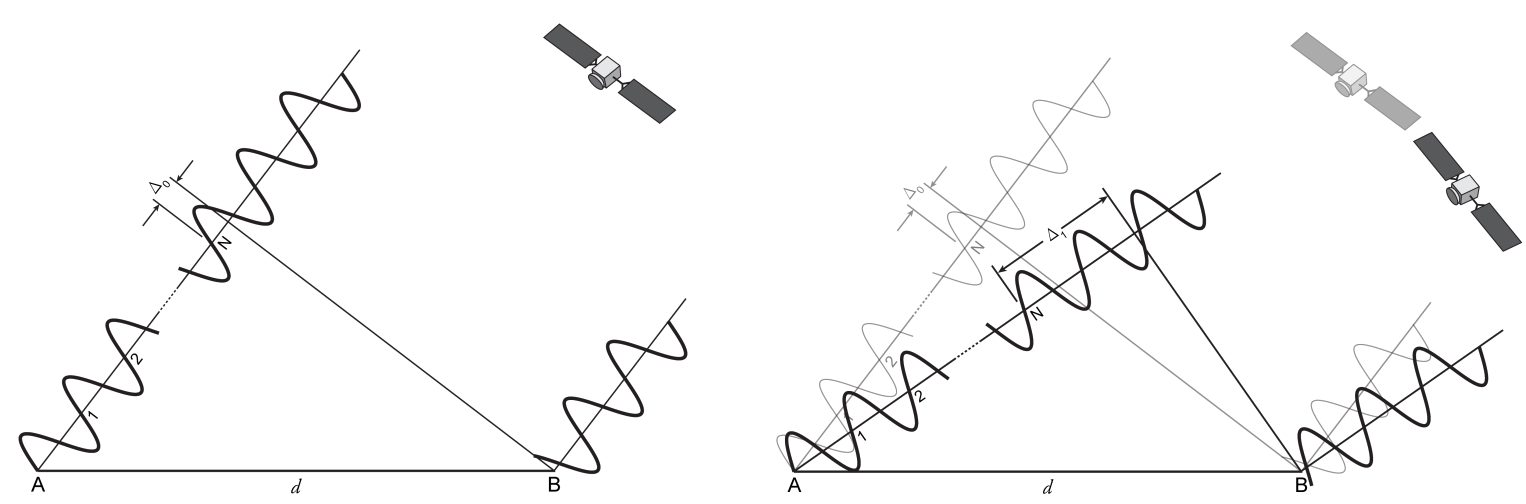

Figure 2.3: Simplified Relative Positioning Example. The image on the left is at the initial time $t_{0}$, and the image on the right is at a later time $t_{1}$, after the satellite has moved. These images are based on Figure 7.1 in $[10]$.

The relative positioning problem of determining $d$ is a matter of resolving the integer ambiguity $N$. If the geometry between the antennas and the satellite remain fixed, the carrier phase difference $\Delta_{0}$ is unchanged, and it becomes difficult to get a unique estimate of $N$. At a later time $t_{1}$, the satellite does move from an elevation angle of $\theta_{0}$ to $\theta_{1}$ relative to the stationary antennas. Assuming the carrier is constantly tracked by both antennas during this satellite motion, the measured difference in carrier phase is now the known sum of a full cycle and a fractional cycle $\Delta_{1}$, and a constant but unknown number of full cycles $N$. The baseline distance at this time instant is now given by

$$
d \cos \left(\theta_{1}\right)=\lambda\left(\Delta_{1}+N\right)
$$

For convenience, we define $d^{\prime}=d / \lambda$. We now have two equations to solve for the two unknowns: $d^{\prime}$ and $N$.

$$
\begin{aligned}
& d^{\prime} \cos \left(\theta_{0}\right)=\Delta_{0}+N \\
& d^{\prime} \cos \left(\theta_{1}\right)=\Delta_{1}+N
\end{aligned}
$$


which is state-space form is

$$
\left[\begin{array}{cc}
\cos \left(\theta_{0}\right) & -1 \\
\cos \left(\theta_{1}\right) & -1
\end{array}\right]\left[\begin{array}{l}
d^{\prime} \\
N
\end{array}\right]=\left[\begin{array}{c}
\Delta_{0} \\
\Delta_{1}
\end{array}\right]
$$

If there was no appreciable difference between $\theta_{0}$ and $\theta_{1}$, then measurements of $\Delta_{0}$ and $\Delta_{1}$ are nearly identical, and the two equations in (2.13) are nearly identical. The second measurement does not offer additional information, and the two equations are linearly dependent. We gain more insight by writing the solution to $(2.13)$ which is

$$
\left[\begin{array}{l}
d^{\prime} \\
N
\end{array}\right]=\frac{1}{\left(\cos \left(\theta_{1}\right)-\cos \left(\theta_{0}\right)\right)}\left[\begin{array}{cc}
-1 & 1 \\
-\cos \left(\theta_{1}\right) & \cos \left(\theta_{0}\right)
\end{array}\right]\left[\begin{array}{c}
\Delta_{0} \\
\Delta_{1}
\end{array}\right]
$$

The multiplier $\left(\cos \left(\theta_{1}\right)-\cos \left(\theta_{0}\right)\right)^{-1}$ in Eq. (2.14) indicates that as the difference in $\theta_{0}$ and $\theta_{1}$ become smaller, the solution becomes less valid. Moreover, small errors in the measurement of $\Delta_{0}$ and $\Delta_{1}$, would result in big errors in the estimates of $d^{\prime}$ and $N$. If we can wait for the satellite to move substantially, then we can get proper estimates of $d^{\prime}$ and $N$. We refer to this significant change in receiver-satellite geometry as providing geometric diversity.

\subsubsection{Point Positioning Using Carrier Phase Measurements from LEO Satellites}

When GPS was the sole provider of practical satellite navigation, geometric diversity was obtained for precise positioning through techniques requiring a stationary reference station, and long initialization times. Improvements in satellite orbit determination, and the emergence of new GNSS and megaconstellations, have laid the groundwork for achieving geometric diversity faster, therefore enabling fast cycle ambiguity estimation with less constraints on the user.

Using signals from LEO satellites is not a new concept. The previous discussion of prior work in section 1.2 recognized some outstanding contributions to this topic. However, today's circumstances are different. We face a paradigm shift where imminent mega-constellations have the potential to increase the number of operational satellites by at least tenfold[5], and the surge of autonomous systems, partic- 
ularly ground vehicles, require centimeter-level positioning in GNSS-challenged environments. There is significant potential to improve GNSS navigation performance by using these mega-constellations.

\subsection{Navigation Performance Metrics}

In this final section for Chapter 2, we define four navigation performance metrics: accuracy, integrity, continuity, and availability, and their role in the performance analysis. These four metrics are defined for aircraft precision approach and landing in [23]. Accuracy is a measure of deviation from absolute truth. This is different from precision, which is a measure of statistical variability. Integrity is a measure of trust in sensor information. This metric is better understood by defining its "opposite" which is integrity risk. Integrity risk is the probability of undetected faults causing unacceptably large positioning errors [15]. Continuity is a system's ability to operate without unscheduled interruptions [15]. Availability is a measure of a system's ability to meet the accuracy, integrity, and continuity requirements.

This work deals with fault-free availability. In this context, a fault refers to any abnormal condition which may lead to a navigation system's failure to perform as intended. Undetected faults pose integrity risks, while detected faults cause loss of continuity. In this preliminary analysis, we are not too concerned with the conflicting requirements of integrity and continuity. Instead, we focus on the ability of the system to achieve a required integrity risk requirement in the absence of system faults for a given period of operation, called fault-free availability. 


\section{Chapter 3}

\section{GNSS-Mega-Constellation System \\ Overview, and Measurement Error Models}

The previous chapter on GNSS fundamentals sets the stage for the augmentation presented in this chapter. Recall that code phase tracking gives an unambiguous estimate of the user position that is accurate to meter-level at best, and in order to get centimeter-level accuracy we need carrier phase measurements that can be tracked to 0.05 cycle $(1 \mathrm{~cm})$, but are burdened with cycle ambiguities. In Section 2.8 it was shown that as long as the carrier is continuously tracked, these cycle ambiguities remain unchanged and can be estimated by taking measurements at well-spaced epochs where there is enough change in satellite geometry. The time consuming cycle ambiguity estimation process for GNSS satellites at medium-Earth orbit (MEO) have limited the use of carrier phase navigation in timesensitive transportation applications. Resurgent interest in deploying large constellations of low-Earth orbit (LEO) communication satellites called mega-constellations has the potential to quickly provide the geometric diversity needed to estimate cycle ambiguities.

This chapter first describes two alternative mega-constellation-augmented GNSS (GNSS-MC) system designs: GNSS-OneWeb and GNSS-Boeing. Since these mega-constellations are primarily used for communication, assumptions are made regarding the ground, space, and user segments that would 
allow these LEO satellites to be used for navigation. There are many configurations possible, and it is important to note that the framework described here is an example. The bulk of the chapter is devoted to the discussion of measurement equations and error models, which are two very important aspects of quantifying the navigation performance of these combined systems. Snapshot error models for GNSS are well established, but error models over time for GNSS and for LEO are not as well understood. Special care has been taken to modify these error models to be applicable for LEO satellite signals. By the end of this section, the essential equations will have been defined and ready to be integrated into a positioning algorithm. Lastly, we examine the process of deriving error models using actual data. The final section describes and implements a methodology for high-integrity two-line element (TLE) error modeling for two example orbital regimes: medium-Earth orbit (MEO) and geostationary Earth orbit (GEO) [9]. The Two-Line Element or TLE is a specific data format for a set of parameters describing an Earth-orbiting object for a specific point in time. TLEs will not be used for the performance analysis, but we will be using as reference, error models that are obtained using the method that will be presented. By looking at the methodology, we illustrate an important building block of integrity analysis called overbounding theory. Using overbounding theory, probabilistic bounds on non-Gaussian error distributions can be achieved $[24,25]$.

\subsection{Envisioned GNSS-Mega-Constellation System Overview}

The LEO mega-constellations will act as stand-alone GNSS, and have all the capabilities necessary to broadcast their own navigation message. We assume a dedicated network of ground stations similar to GNSS that would monitor the LEO spacecraft and make the satellite position and time synchronization information available to users. We assume that there are no inter-satellites links. Figure 3.1 depicts the aforementioned configuration. 


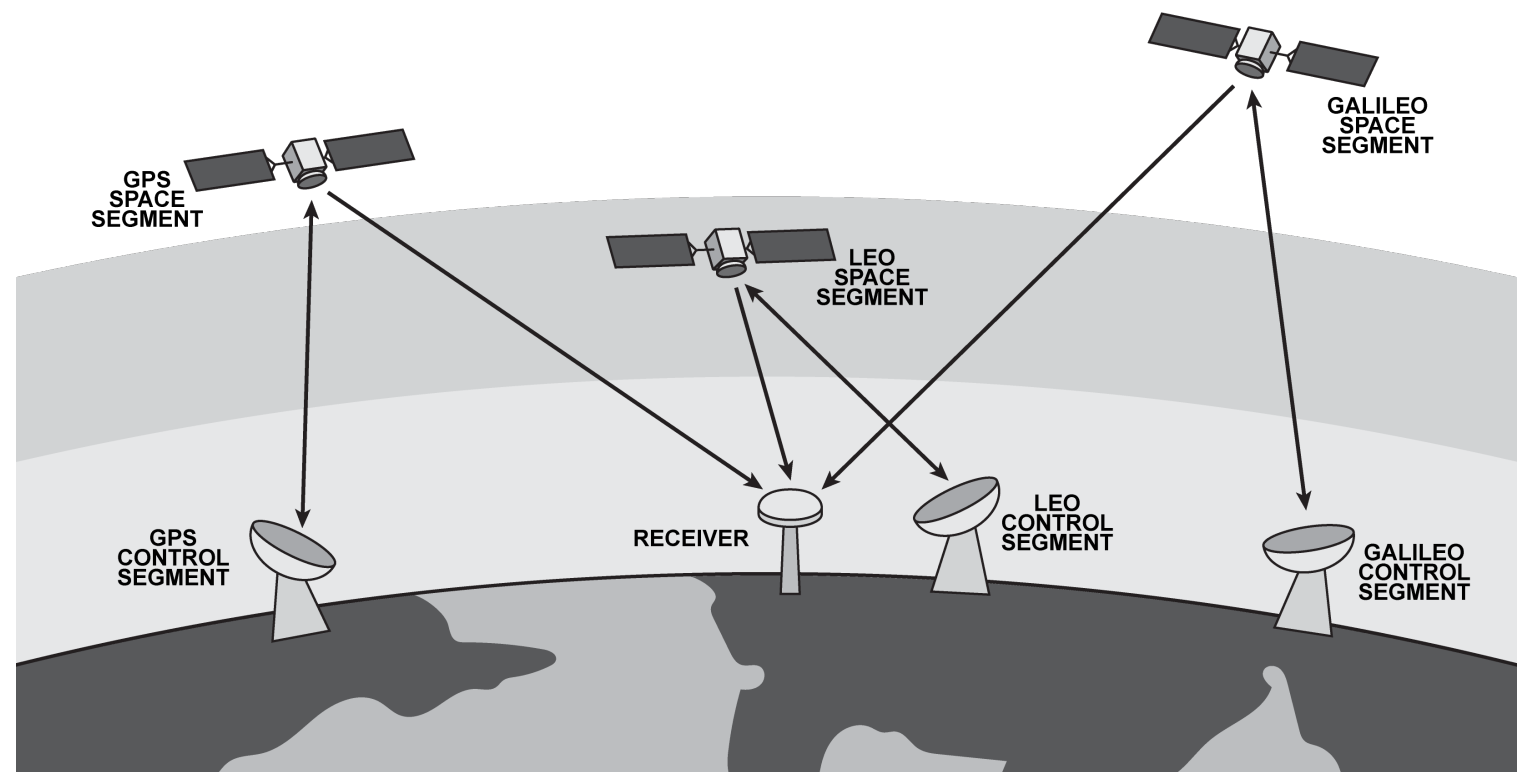

Figure 3.1: Mega-Constellation-Augmented GNSS Configuration. The mega-constellations have their own operational control segment with a dedicated network of ground stations monitoring the LEO spacecrafts. Users can get measurements from both GNSS and LEO satellites to estimate their position.

\subsubsection{Envisioned Space Segment}

The space segments for GPS and Galileo were discussed in Chapter 2. Here we discuss the two megaconstellations chosen to augment GNSS: OneWeb and Boeing.

The impending OneWeb satellite constellation aims to provide global satellite Internet broadband services everywhere on Earth by 2027 [26]. Since the first reports about OneWeb’s (formerly called WorldVu) plans to build a "very large constellation," the number of proposed satellites has fluctuated between 400 and 2620. In this work, we take the number of satellites to be 720 in accordance with [27]. OneWeb's 720-satellite constellation utilizes a near-polar Walker Star configuration. The constellation design allows satellite coverage at all latitudes at all time. Since OneWeb satellites generally move in the north-south direction, we expect preferential navigation performance in the north-south direction. OneWeb's satellites will communicate via the Ka- and $\mathrm{Ku}$ - spectrum bands. The first six OneWeb satellites were successfully launched in February 2019 [28]. 
Boeing has had plans to deploy a more massive 2956-satellite constellation in low-Earth orbit, although there has not been any development since they filed for spectrum allocation with the Federal Communications Commission (FCC) in 2016 [29]. Boeing intends to use V-and C-bands [30]. In this work, we assume the satellite configuration for Boeing depicted in 3.3. In this figure, the three colors distinguish three distinct constellation configurations. Boeing's 2956-satellite constellation has both near-polar and inclined orbits. This contrast with OneWeb's purely near-polar constellation, along with the four-fold increase in satellite count enhances the study region of our sensitivity analysis in Chapter 5.

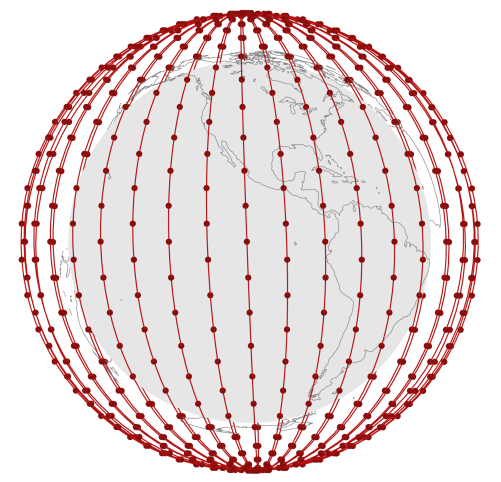

Figure 3.2: OneWeb Constellation. The 720-satellite OneWeb constellation used in the simulations has 18 orbital planes inclined at $87.9^{\circ}$ at $1200 \mathrm{~km}$ altitude. This configuration is based on information found in [27].

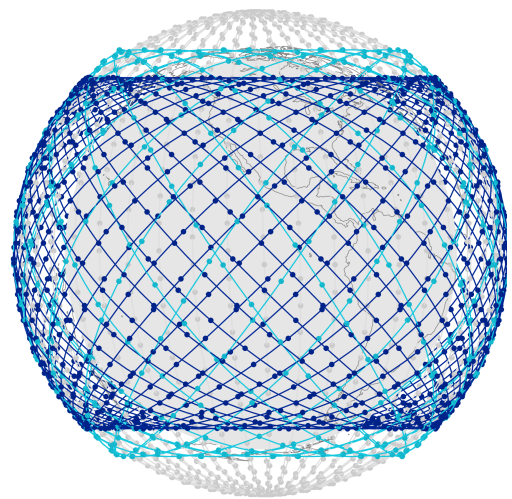

Figure 3.3: Boeing Constellation. The 2956satellite Boeing constellation used in the simulations has 1225 satellites in 35 planes inclined at $45^{\circ}$, and 576 satellites in 18 planes inclined at $55^{\circ}$ at 1200 $\mathrm{km}$ altitude. Additionally, there are 1155 satellites in 21 more orbital planes inclined at $88^{\circ}$ at 1000 $\mathrm{km}$ altitude. This is based on information in [30].

Unless differential corrections are available, dual-frequency signals are essential to mitigate the impact of ionospheric delay. We assume dual-frequency carrier signals from mega-constellation satellites. Both OneWeb and Boeing have indicated capability of providing dual-frequency signals [31, 30].

Satellites at low-Earth orbit "see" a smaller patch of Earth than satellites at medium-Earth orbit, referred to as a smaller satellite footprint. Satellite footprints depend on Earth terrain, and antenna gain patterns. Ideally, constellation orbits are designed so that the footprint of each satellite overlap, and the constellation provides continuous global coverage at all times. We assume no limit on the LEO spacecraft footprint due to narrow satellite antenna gain pattern. 
In this work, we investigate the navigation performance of three configurations: GNSS only, GNSSOneWeb, and GNSS-Boeing. These three configurations were chosen to each represent a navigation system without augmentation (GNSS only), an average case of mega-constellation-augmented GNSS (GNSS-OneWeb), and an extreme case of mega-constellation-augmented GNSS (GNSS-Boeing). We will refer back to figures in this section when we discuss the sensitivity analysis in Chapter 5.
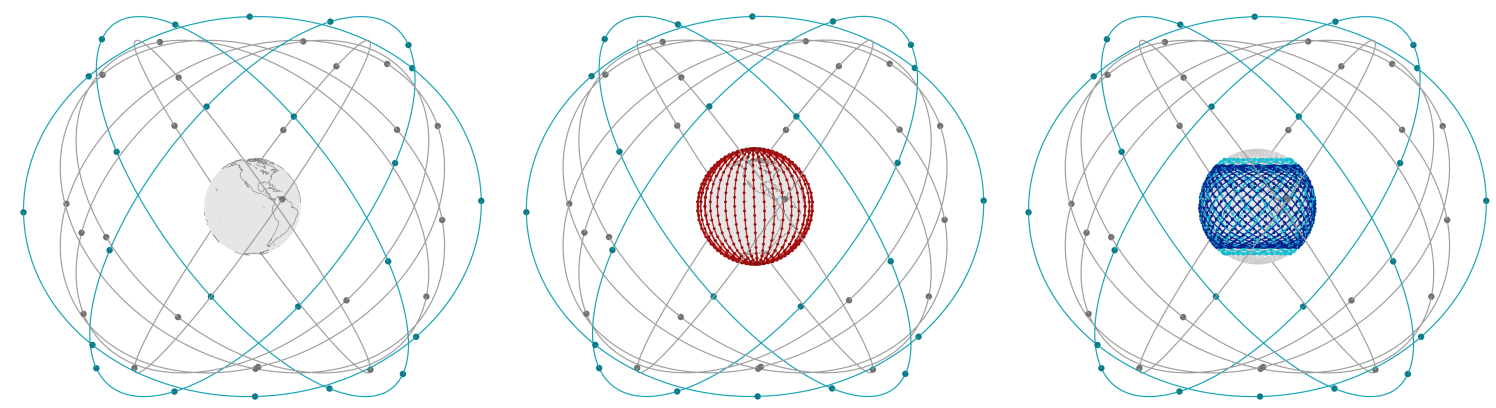

Figure 3.4: Mega-Constellation-Augmented GNSS Configurations: (left) GNSS only, (center) GNSSOneWeb, (right) GNSS-Boeing. GPS is in gray, Galileo is in teal, OneWeb is in red, and the three sets of constellations for Boeing are shown in light gray, cyan, and dark blue.

\subsubsection{Envisioned Control Segment}

A dedicated ground control segment is envisioned to perform orbit and clock determination for the LEO mega-constellations. The ground monitoring stations can be collocated with data monitoring stations needed for the communications function of the satellite. OneWeb has plans for more than fifty ground stations globally for communication [27, 32]. Mega-constellation LEO satellite orbit and clock offsets are estimated and monitored by a dedicated network of ground stations, uploaded to the satellites, and broadcast to users. We assume that the mega-constellations are time-synchronized with GPS. The clocks aboard LEO communication spacecraft are not expected to have the same standard as those aboard GNSS satellites. However, these satellites are closer to Earth, and high throughput data links can be established between the satellites and the control segment [31]. Higher data rates and more frequent updates to the broadcast message could potentially compensate for the not-so-accurate clocks aboard the LEO satellites and the less established measurement error models. 


\subsubsection{Envisioned User Segment}

We assume that the user is equipped with a dual-frequency receiver capable of receiving and processing measurements from GNSS and mega-constellations. The dual frequency capability is an important simplifying assumption that eliminates the impact of ionospheric errors. User equipment is assumed to have the requisite receiving and computing capabilities that would allow it to implement the estimation algorithm discussed in Chapter 4.

\subsection{Nominal Measurement Error Models}

This section deals with the measurement error models for the combined system, based on the notional architecture outlined in section 3.1. We assume dual-frequency measurements to eliminate the impact of ionospheric errors. The remaining errors including tropospheric delay, orbit, clock, multipath errors, and receiver noise are modeled conservatively over time as recommended in [8]. Under nominal faultfree conditions, the linearized ionosphere-error-free carrier phase and code measurement equations for satellite $i$ at time $k$ respectively are

$$
\begin{aligned}
{ }^{i} \phi_{k} & =-{ }^{i} \mathrm{e}_{k}^{\mathrm{T}} \mathbf{x}_{k}+\tau_{k}+{ }^{i} \eta+{ }^{i} \mathscr{E}_{E, k}+{ }^{i} \mathscr{E}_{T, k}+{ }^{i} \mathscr{E}_{M, \phi, k}+{ }^{i} \mathscr{E}_{R, \phi, k} \\
{ }^{i} \rho_{k} & =-{ }^{i} \mathrm{e}_{k}^{\mathrm{T}} \mathbf{x}_{k}+\tau_{k}+{ }^{i} \mathscr{E}_{E, k}+{ }^{i} \mathscr{E}_{T, k}+{ }^{i} \mathscr{E}_{M, \rho, k}+{ }^{i} \mathscr{E}_{R, \rho, k}
\end{aligned}
$$

where

${ }^{i} \phi_{k} \quad$ is the carrier phase measurement for satellite $i$,

${ }^{i} \rho_{k} \quad$ is the code phase measurement for satellite $i$,

${ }^{i} \mathrm{e}_{k}$ is the $3 \times 1$ line-of-sight vector from the satellite to the user in North-East-Down (NED),

$\mathbf{x}_{k} \quad$ is the user position in NED,

$\tau_{k} \quad$ is the receiver clock offset,

${ }^{i} \eta \quad$ is the carrier phase cycle ambiguity, (there is no subscript $k$ because it is constant over time)

${ }^{i} \mathscr{E}_{E, k}$ is the satellite clock and orbit ephemeris error,

${ }^{i} \mathscr{E}_{T, k}$ is the residual tropospheric error,

${ }^{i} \mathscr{E}_{M, k}$ is the multipath error, and

${ }^{i} \mathscr{E}_{R, k}$ is the receiver noise error.

GPS and Galileo provide carrier and code phase measurements, while the OneWeb and Boeing LEO constellations provide carrier phase measurements. The treatment for each of these error terms, as well 
as the parameters for ground vehicle application are provided in the subsections that follow.

\subsubsection{Satellite Orbit and Clock Error}

The satellite orbit and clock error is modeled using a first-order Gauss Markov Process (GMP) with Markov process correlation time constant $T_{E}$, and driving noise $\nu_{E, k}$. It is assumed that there is no correlation between measurements from different satellites, and that the clocks on the LEO satellites are synchronized with GPS. The error due to satellite orbit and clock for satellite $i$ at time $k$ is

$$
{ }^{i} \mathscr{E}_{E, k}=e^{-T_{K} / T_{E}}{ }^{i} \mathscr{E}_{E, k-1}+\nu_{E, k} \quad, \quad \nu_{E, k} \sim N\left(0, \sigma_{E}^{2}\left(1-e^{-2 T_{K} / T_{E}}\right)\right)
$$

where $T_{K}=t_{k}-t_{k-1}$, and $\sigma_{E}$ is the GMP standard deviation. Based on the recommendations in $[33,8]$, the GMP correlation time constant and standard deviation for the specific application of ground vehicles are given as

$$
\begin{aligned}
\sigma_{E, M E O} & =1 \mathrm{~m}[33] \\
T_{E, M E O} & =7200 \mathrm{~s}[8]
\end{aligned}
$$

The LEO satellite clocks and orbit ephemeris are assumed to be updated once per orbit for a LEO orbital period of 100 minutes. This assumption coupled with a 50\% increase in the GMP standard deviation gives us the following error parameters for LEO spacecraft satellite clock and orbit ephemeris error

$$
\begin{aligned}
\sigma_{E, L E O} & =1.5 \mathrm{~m} \\
T_{E, L E O} & =6000 \mathrm{~s}(100 \mathrm{~min})
\end{aligned}
$$

These values for the LEO orbit and clock error have not been verified, but are recognized as a good starting point based on discussions in [5].

\subsubsection{Residual Tropospheric Error}

Most of the error caused by tropospheric delay is removed using a model of the troposphere. The residual tropospheric error is treated as a first-order GMP. The error variation caused by signals traveling through the troposphere at different angles is accounted for by using a mapping function described in [8]. The 
resulting error contribution for satellite $i$ at time $k$ is given by

$$
{ }^{i} \mathscr{E}_{T, k}={ }^{i} c_{T, k}{ }^{i} \zeta_{T, k}
$$

where the elevation-dependent tropospheric correction mapping function [8] is

$$
{ }^{i} c_{T, k}=\frac{1.001}{\sqrt{0.002001+\left(\sin \left({ }^{i} \theta_{k}[\mathrm{rad}]\right)\right)^{2}}}
$$

for satellite elevation angles ${ }^{i} \theta_{k} \geq 4^{\circ}$. The residual tropospheric error at zenith is modelled as

$$
{ }^{i} \zeta_{T, k}=e^{-T_{K} / T_{T}}{ }^{i} \zeta_{T, k-1}+\nu_{T, k} \quad, \quad \nu_{T, k} \sim N\left(0, \sigma_{T}^{2}\left(1-e^{-2 T_{K} / T_{T}}\right)\right)
$$

The GMP correlation time constant and standard deviation are retrieved from [34] and are given as

$$
\begin{aligned}
& \sigma_{T}=0.12 \mathrm{~m} \\
& T_{T}=1800 \mathrm{~s}
\end{aligned}
$$

where $0.12 \mathrm{~m}$ is the tropospheric vertical error standard deviation given in A.4.2.5 of [34]. Since the tropospheric model characterizes the airmass encountered by the signal, the model is the same for GNSS and mega-constellations. The difference in satellite motion is captured in the elevation-dependent mapping coefficient, Eq. (3.8).

\subsubsection{Multipath Error}

In order to account for the time-correlation caused by unwanted signal reflections reaching the receiver antenna, the multipath error is modeled as a first-order GMP, multiplied by the elevation-dependent mapping function given in [34]. The multipath error contribution for satellite $i$ at time $k$ is given by

$$
\begin{aligned}
{ }^{i} \mathscr{E}_{M, \phi, k} & ={ }^{i} c_{M, k}{ }^{i} \zeta_{M, \phi, k} \\
{ }^{i} \mathscr{E}_{M, \rho, k} & ={ }^{i} c_{M, k}{ }^{i} \zeta_{M, \rho, k}
\end{aligned}
$$

where the elevation-dependent multipath mapping function is

$$
{ }^{i} c_{M, k}=(10) c_{I F}\left(0.13+0.53 e^{\left(-^{i} \theta_{k}[\mathrm{deg}] / 10\right)}\right) .
$$


The factor 10 comes from using raw unsmoothed code measurements which can be up to 10 times noisier than carrier-smoothed code measurements [8]. The ionosphere-free measurement combination multiplier, $c_{I F}$ is computed with L1 and L5 and given by the equation

$$
c_{I F}=\sqrt{\frac{f_{L 1}^{4}+f_{L 5}^{4}}{\left(f_{L 1}^{2}-f_{L 5}^{2}\right)^{2}}}
$$

where $f_{L 1}$ and $f_{L 5}$ are the frequencies for L1 and L5 respectively. The GMP term in Eq. (3.12) for carrier and code measurements

$$
\begin{aligned}
{ }^{i} \zeta_{M, \phi, k} & =e^{-T_{K} / T_{M} i} \zeta_{M, \phi, k-1}+\nu_{M, \phi, k} & & \nu_{M, \phi, k} \sim N\left(0, \sigma_{M, \phi}^{2}\left(1-e^{-2 T_{K} / T_{M}}\right)\right) \\
{ }^{i} \zeta_{M, \rho, k} & =e^{-T_{K} / T_{M} i} \zeta_{M, \rho, k-1}+\nu_{M, \rho, k} & & \nu_{M, \rho, k} \sim N\left(0, \sigma_{M, \rho}^{2}\left(1-e^{-2 T_{K} / T_{M}}\right)\right) .
\end{aligned}
$$

GNSS multipath error has been modeled for automotive applications in [35], where values for the correlation time constant and pseudorange bounding standard deviation can be used as reference. Taking these values into account ${ }^{1}$, the GMP standard deviation and correlation time constant for GNSS are

$$
\begin{aligned}
\sigma_{M, M E O, \rho, k} & =1.5 \mathrm{~m} \\
\sigma_{M, M E O / L E O, \phi, k} & =\left(\frac{1}{100}\right) 1.5 \mathrm{~m} \\
T_{M, M E O} & =158 \mathrm{~s} .
\end{aligned}
$$

The multiplier $100^{-1}$ accounts for the order of magnitude difference in multipath error for code and carrier. We assume a similarly derived GMP standard deviation for the LEO constellations, but deriving the time constant is trickier. LEO satellites move faster, so the correlation time constant needs to be appropriately scaled down. For this, we use the ratio of angular velocities between GNSS and megaconstellations relative to a user on Earth. We use as reference the angular velocity ratio derived in [36] between GPS and a constellation of LEO satellites called Iridium, which was evaluated every 30s over a 3-day period to compute for the average ratio of 30. The resulting GMP time constant for LEO

\footnotetext{
${ }^{1} \mathrm{~A}$ range of values are provided in [35] for a variety of test cases. We take the largest bounding standard deviation and longest correlation time constant.
} 
multipath error is

$$
T_{M, L E O}=6 \mathrm{~s} \approx\left(\frac{1}{30}\right)(158) \mathrm{s} .
$$

Later we will find that this time constant can be much smaller than the batch measurement sampling rate in our example implementation. This means that multipath error does not appear correlated in time for LEO carrier phase measurements. This is because relative to a user on Earth, LEO satellites traverse the sky much faster than MEO satellites, and the resulting error due to multipath appears uncorrelated in time.

\subsubsection{Receiver Noise Error}

Receiver noise is modeled as Gaussian white noise with elevation-dependent standard deviation, and is computed as

$$
\begin{aligned}
& { }^{i} \mathscr{E}_{R, \phi, k}={ }^{i} \nu_{R, \phi, k} \quad, \quad{ }^{i} \nu_{R, \phi, k} \sim N\left(0,{ }^{i} \sigma_{R, \phi}^{2}\right) \\
& { }^{i} \mathscr{E}_{R, \rho, k}={ }^{i} \nu_{R, \rho, k} \quad, \quad{ }^{i} \nu_{R, \rho, k} \sim N\left(0,{ }^{i} \sigma_{R, \rho}^{2}\right)
\end{aligned}
$$

Elevation-dependent receiver noise error models are formulated for airborne aircraft using carrier-smoothed code measurements from GNSS in [33]. Error due to raw unsmoothed code can be up to 10 times greater [8]. The effect of receiver noise is assumed to be the same for MEO and LEO measurements. The equations for these are

$$
\begin{aligned}
{ }^{i} \sigma_{R, M E O, \rho, k} & =(10) c_{I F}\left(0.15+0.43 e^{\left(-{ }^{i} \theta_{k}[\mathrm{deg}] / 6.9\right)}\right) \\
{ }^{i} \sigma_{R, M E O / L E O, \phi, k} & =\left(\frac{1}{100}\right)^{i} \sigma_{R, M E O, \rho, k}
\end{aligned}
$$

\subsubsection{Summary of Error Parameters}

The values allocated for the measurement error parameters for ground vehicle applications are summarized in table 3.1 for GNSS (GPS/Galileo) and mega-constellations (OneWeb/Boeing). These error models will have to be further evaluated using experimental data. In particular, data processing is necessary to validate the LEO measurement error models discussed in this chapter. 
GNSS-MC SYSTEM OVERVIEW, AND MEASUREMENT ERROR MODELS

Table 3.1: Summary of GMP Error Model Parameters

\begin{tabular}{|c|c|c|c|}
\hline GMP & Carrier/Code & GPS/Galileo & OneWeb/Boeing \\
\hline \multicolumn{4}{|c|}{ Clock and Orbit Ephemeris } \\
\hline \multirow{3}{*}{$\begin{array}{l}\sigma_{E} \\
T_{E} \\
\end{array}$} & & $1 \mathrm{~m}$ & $1.5 \mathrm{~m}$ \\
\hline & & $7200 s$ & $6000 s$ \\
\hline & & \multicolumn{2}{|c|}{ Troposphere } \\
\hline \multirow{3}{*}{$\begin{array}{l}\sigma_{T} \\
T_{T} \\
\end{array}$} & & Eq. $(3.10)$ & Eq. $(3.10)$ \\
\hline & & $1800 \mathrm{~s}$ & $1800 \mathrm{~s}$ \\
\hline & & \multicolumn{2}{|c|}{ Multipath } \\
\hline \multirow{2}{*}{$\sigma_{M}$} & $\phi$ & Eq. $(3.17)$ & Eq.(3.17) \\
\hline & $\rho$ & Eq.(3.16) & \\
\hline \multirow[t]{2}{*}{$T_{M}$} & & $158 \mathrm{~s}$ & $6 s$ \\
\hline & & \multicolumn{2}{|c|}{ Receiver Noise } \\
\hline \multirow{2}{*}{$\sigma_{R}$} & $\phi$ & Eq. $(3.22)$ & Eq. (3.22) \\
\hline & $\rho$ & Eq. $(3.21)$ & \\
\hline
\end{tabular}

\subsection{Measurement Error Modelling Using TLE Data}

In this section, we describe the development of measurement error models using real data. The Joint Space Operations Center (JSpOC) provides spacecraft ephemerides in the form of TLEs, which represent the most comprehensive catalogue of space objects readily accessible to the public. In this section, we compare 20 years of TLE data with precise ephemerides for GPS and geostationary Earth-orbiting (GEO) Wide Area Augmentation System (WAAS) satellites [9]. Based on this data, we derive highintegrity error models using overbounding theory to determine probabilistic bounds on the radial, along-track, and cross-track positioning errors. While TLEs are not intended for high-accuracy, highintegrity navigation, they have been used in navigation before [4], and are relevant to Space Situational Awareness. The main steps of the error evaluation method described below applies to other sources of orbit ephemeris data $[37,38]$.

Overbounding theory is a building block of integrity evaluation $[25,24]$. It leverages the fact that for 
safety, risk estimates do not need to be exact, but must upper-bound the actual risk. Thus, a finite set of measurements is sufficient to characterize a sensor (in this case the TLE database) with quantifiable integrity. The actual implementation of overbounding theory on error data is tackled in the discussion of Section 3.3.2.

In addition, TLE errors are unlikely to follow a known distribution. In this regard, overbounding theory can be exploited [25, 24], as it currently is in aviation navigation applications [39, 33, 15]. First, overbounding provides the means to model empirical TLE data using familiar parametric distributions; namely Gaussian functions. Second, if errors for TLE ephemerides can be over-bounded, then orbit determination errors will be bounded as well, provided that the orbit estimator is linear or linearized.

The TLE error data is described first in Section 3.3.1. We then use Gaussian overbounding to establish a high-integrity, compact, parametric model of TLE data for GPS satellites in Section 3.3.2, and for GEO WAAS satellites in Section 3.3.3.

\subsubsection{TLE Error Data}

In order to evaluate TLE errors, we consider spacecraft at known locations. Both GPS and WAAS satellites have a well maintained history of ephemerides with known accuracy [37, 19, 38] orders of magnitude higher than TLEs currently documented in the literature [40, 41, 42, 43]. Each GPS and WAAS satellite is identified by their unique pseudo-random noise (PRN) signal numbered from 1 to 32 for GPS, and 131 to 138 for WAAS.

TLE errors for thirty GPS satellites are plotted in Figure 3.5. There were 146,660 total TLE error data points. Magnitudes of along-track errors are significantly larger than errors in the other two directions. An apparent shrinkage of error spread occurs sometime after July 2012, and again after January 2015. 


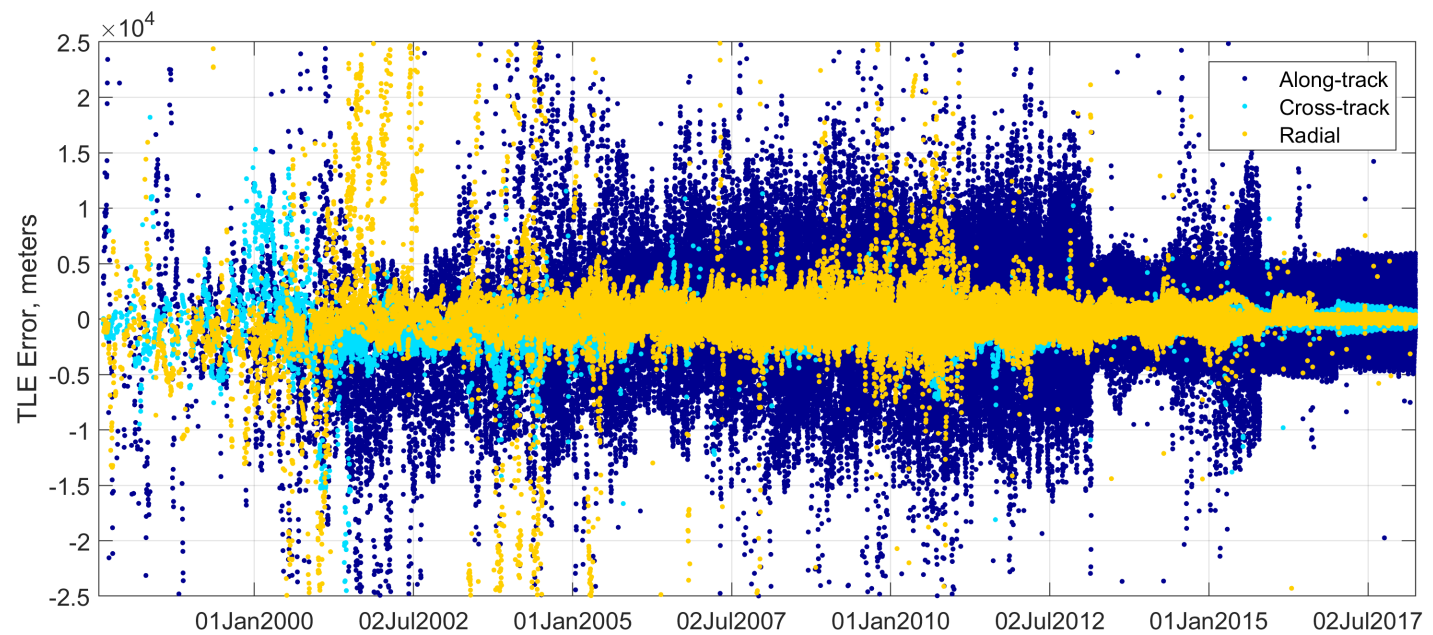

Figure 3.5: MEO GPS TLE Errors. This figure plots the error between TLEs and GPS broadcast ephemerides for PRNs 1-3, 5-17, and 19-32, from July 23, 1997 to March 31, 2018, expressed in the radial, along-track, and cross-track reference frame.

TLE errors sometimes exceed the 25,000 meters y-axis scale used in Figure 3.5. Most of the "outlier" data points could easily be identified, for example considering the consistency of TLE orbit parameters with previous TLEs. A detailed TLE error outlier analysis will be performed in future work. In this study, a 20,000 meters threshold is applied. Error values greater than the 20,000-meter hard limit were considered outliers.

The top plot of Figure 3.6 zooms in on the time when the TLE error behavior suddenly changes to form a more consistent pattern. There is more consistent behavior over recent years, with most TLE along-track errors ranging from $-15 \mathrm{~km}$ to $15 \mathrm{~km}$ between March 2013 and October 2015, but staying within -6 to $6 \mathrm{~km}$ from October 2015 to 2018. Error data after October 21, 2015 is interpreted as stationary for statistical error analysis. The bottom plot in Figure 3.6 shows that the time of this sudden change for GPS TLE error coincides with that for WAAS TLE error, which are further discussed in the next paragraph. The analysis in Section 3.3.2 is focused on the period which starts on 21 October 2015, 23:18:33 UTC, and ends on 1 April 2018, 00:00:00 UTC. 

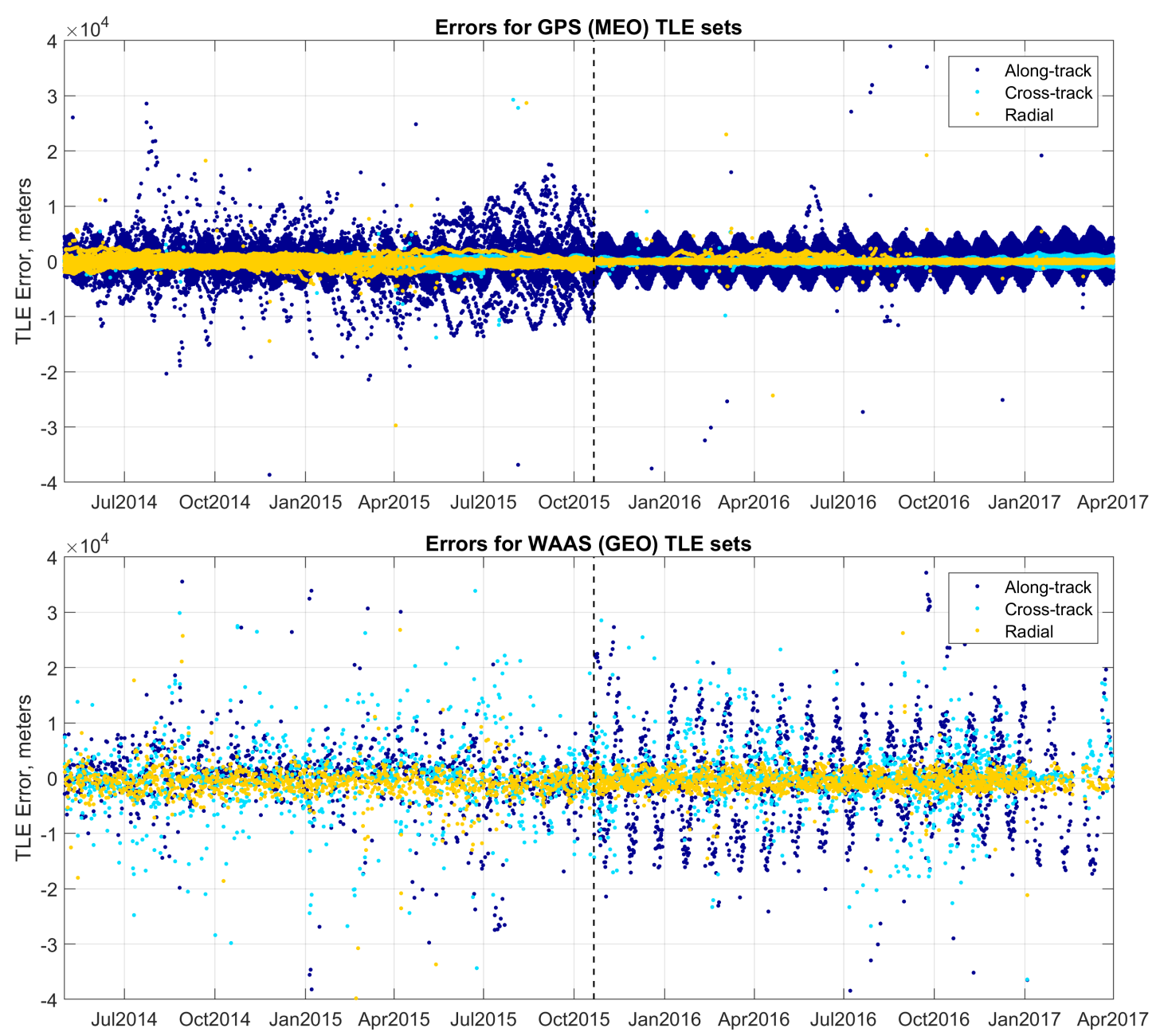

Figure 3.6: MEO GPS and GEO WAAS TLE Error Comparison. The error plot for GPS (top) and WAAS (bottom) TLEs both show an abrupt change in error behavior after October 21, 2015.

TLE errors for WAAS satellites are depicted in Figure 3.7 with a zoomed-in portion shown in the bottom portion of Figure 3.6. There were 5,813 total TLE error data points. Error magnitudes for GEOs are greater than MEO GPS TLE errors. Unlike Figure 3.5, along-track errors here are not as noticeably larger than in the other directions. For this GEO data set, cross-track errors are of the same magnitude as along-track errors. 


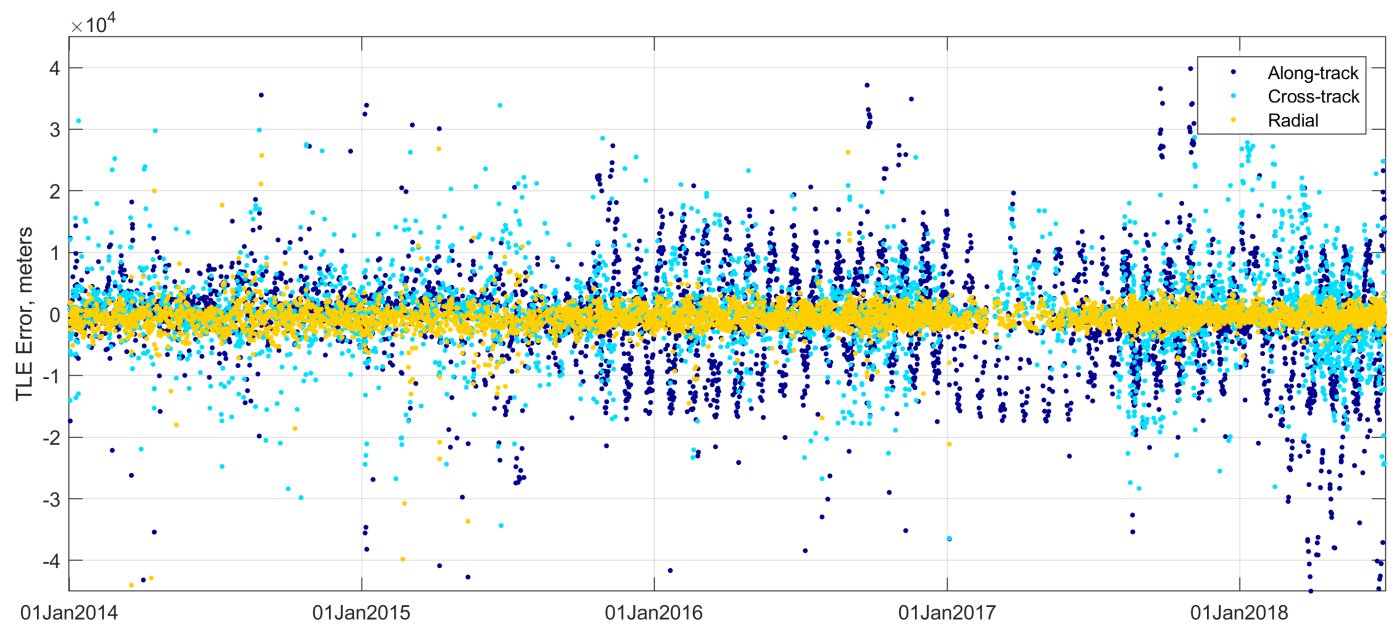

Figure 3.7: GEO WAAS TLE Errors. This figure plots the error between TLEs and the NSTB navigation message for PRNs 131, 133, 135, and 138, from January 1, 2014 to June 30, 2018, expressed in the radial, along-track, and cross-track reference frame.

Similar to GPS TLE errors, the behavior of errors distinctly changes in year 2015. Figure 3.6, displayed on page 45 shows this parallel. For the statistical analysis on TLE errors for WAAS GEO satellites, we exclude errors larger than 40,000 meters. Similar to the preceding MEO TLE error analysis, 40,000 meters is an arbitrary cut-off (of twice the value used for GPS TLE errors).

\subsubsection{Gaussian Overbounding Model of TLE Errors for MEO GPS Satellites}

Gaussian overbounding functions are best represented on quantile-to-quantile (q-q) plots. A q-q plot is used to determine if a set of data comes from a known distribution. As depicted in Figure 3.8, the q-q plot graphs the quantiles of TLE error data (y-axis) against the theoretical quantiles from a standard normal distribution (x-axis). If the distribution of TLE errors was normal, then the sample cumulative distribution curve would appear as a straight line, with slope the sample standard deviation and y-intercept the sample mean. However, TLE errors are not normally distributed.

On a q-q plot, we find a pair of overbounding functions by sandwiching the sample error distribution between two straight lines corresponding to Gaussian overbounding functions. This pair of Gaussian functions provide a conservative probabilistic model that can be used in risk analysis. In Figure 3.8, 
the dashed lines that sandwich the radial, along-track, and cross-track error distributions are the overbounding lines. The slope and y-intercept of these overbounding lines represent the overbounding standard deviation, $\sigma_{o b}$, and bounding biases, $\pm \mu_{o b}$ respectively. The positive bias corresponds to the left overbound (above the actual error curve), and the negative bias to the right overbound. The legend shows the values for these slopes and biases, as well as the sample mean, $\mu_{s}$, and standard deviation, $\sigma_{s}$. Using $\mu_{s}$ and $\sigma_{s}$ for the TLE error model in estimation would be optimistic because they only take into account the core of the distribution. For risk analysis to meet stringent requirements, $\sigma_{o b}$, and $\pm \mu_{o b}$ should be used instead.

Two example overbounding models are presented in Figures 3.8 and 3.9. The one in Figure 3.8 covers the entire sample distribution made of more than $10^{4}$ data samples. If the process is stationary, this model will be valid approximately $99.99 \%$ of the time $\left(1-10^{-4}\right)$. In parallel, in Figure 3.9, we derived an overbounding model that only covers the core of the sample distribution, between \pm 3.3 on the $x$-axis, i.e., between \pm 3.3 standard deviations of the standard normal distribution, which corresponds to a $10^{-3}$ quantile. In Figure 3.9, the values of $\sigma_{o b}$, and $\pm \mu_{o b}$ are much lower than in Figure 3.8, but the model is only valid $99.9 \%$ of the time.
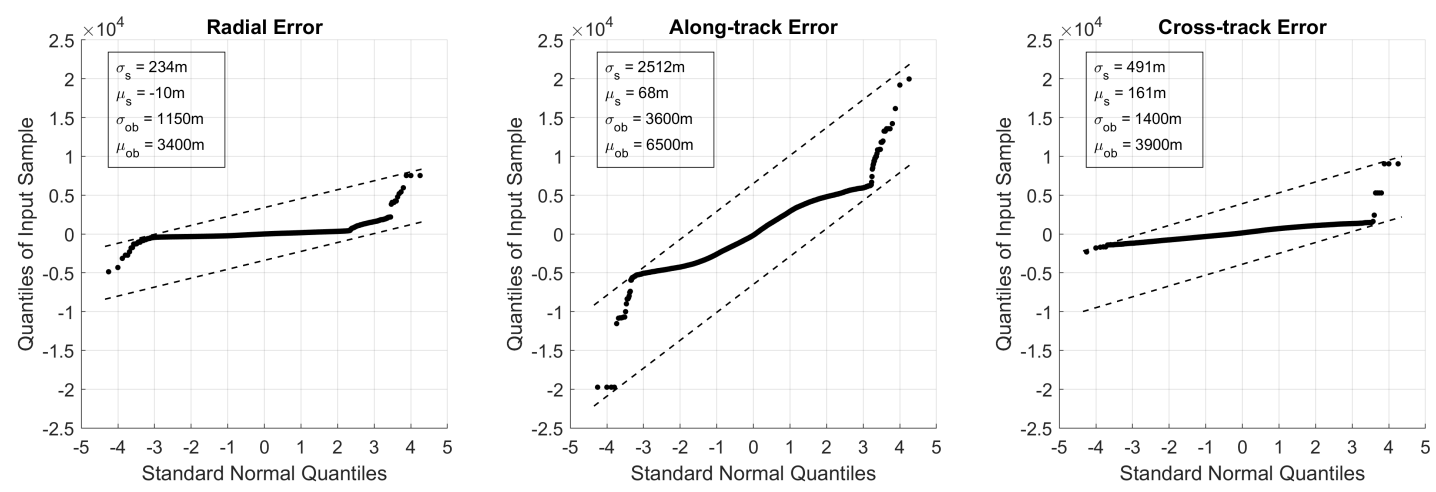

Figure 3.8: MEO GPS Q-Q Plot for 99.99\%. Quantile-to-quantile plots of 99.99\% paired overbounding model for 47,626 GPS TLE error data points from October 21, 2015 to March 31, 2018 

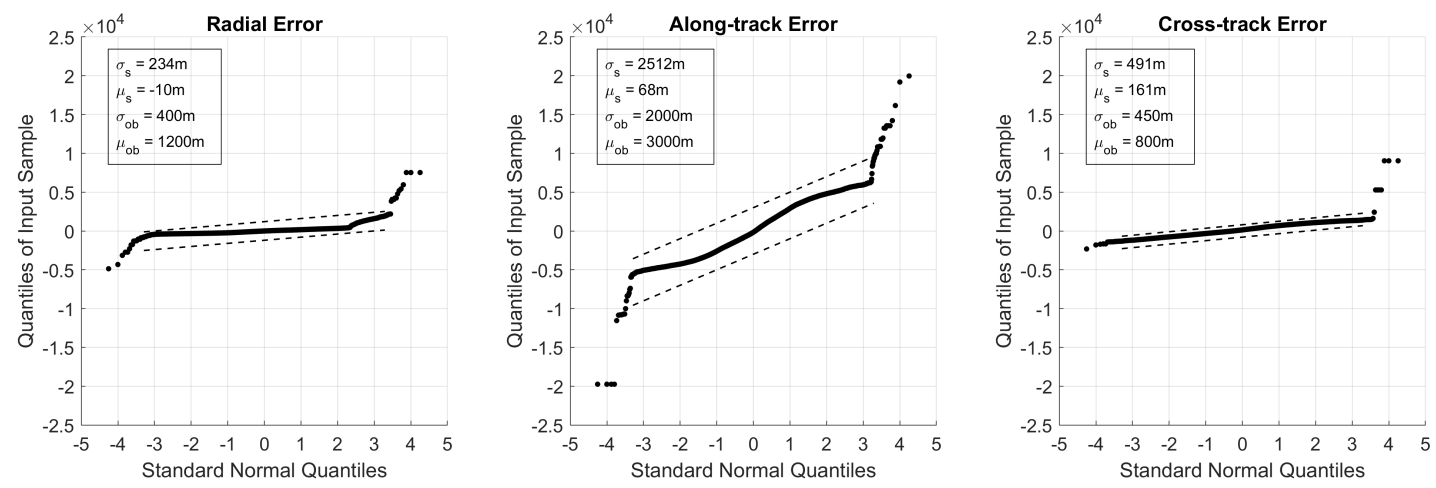

Figure 3.9: MEO GPS Q-Q Plot for 99.9\%. Quantile-to-quantile plots of $99.9 \%$ paired overbounding model for 47,626 GPS MEO TLE error data points from October 21, 2015 to March 31, 2018

\subsubsection{Gaussian Overbounding Model of TLE Errors for GEO WAAS Satellites}

Figure 3.10 depicts the overbounding functions for WAAS TLE errors. Sample error distributions for the radial, along-track, and cross-track directions are all well-behaved without too many stray points on the tails of the distribution. This differs significantly from what was observed for GPS in Figure 3.8, but the number of samples $(3,979)$ is much lower than for GPS $(47,626)$. The overbounding model that covers these more than $10^{3}$ data points will be deemed valid approximately $99.9 \%$ of the time $\left(1-10^{-3}\right)$. Sample standard deviations and means, as well as bounding standard deviations and bounding biases are given in legend in Figure 3.10.
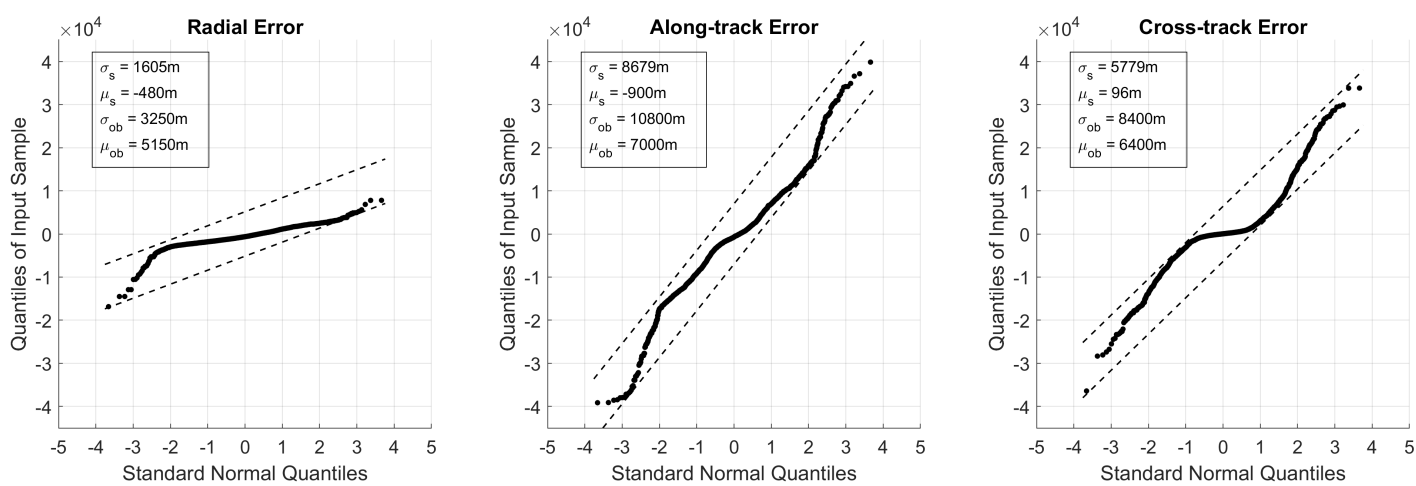

Figure 3.10: GEO WAAS Q-Q Plot for 99.9\%. Quantile-to-quantile plots of 99.9\% paired overbounding model for 3,979 WAAS TLE error points from October 21, 2015 to June 30, 2018 


\section{Chapter 4}

\section{GNSS-Mega-Constellation Positioning}

\section{Algorithm}

In navigation applications, position and velocity can be estimated using a time history of measurements and a mathematical model of the dynamics of the user. A Kalman filter can be used to incorporate measurements from GPS and other sensors to obtain an estimate of user position and velocity [10]. In this work, we focus on estimating the user position, clock bias, and cycle ambiguities using a batch weighted least squares (WLS) approach. This method can be implemented sequentially in a sliding window mechanism, and will leverage fault detection algorithms in future work [44, 45, 46].

In this chapter, we derive a batch weighted least squares algorithm for estimating the user parameters using dual-frequency code and carrier phase measurements from GNSS, and dual-frequency carrier phase measurements from mega-constellations. This algorithm simultaneously estimates the threedimensional user position and receiver clock offset at every time step, and estimates the floating valued carrier phase cycle ambiguities that remain constant, as long as the carrier is tracked continuously. This batch approach leverages two estimation principles: noise averaging and bias observability by exploiting satellite motion. This approach can be implemented in practical applications as long as adequate receiver memory is available to store current and past measurements [47]. In a batch approach, the process 
equation is incorporated into the measurement equation by means of stacking the measurements for all times. The algorithm assumes no knowledge about the user's dynamics, but captures the fact that the carrier is continuously tracked, and as a result the cycle ambiguities remain constant. Even a short lapse in carrier tracking, e.g. due to a momentary obstruction in user-to-satellite line of sight, causes the cycle count to be reinitialized. Also, cycle slips occur, which are a known receiver tracking failure [10]. We assume that there are no unrepaired cycle slips.

\subsection{Batch Measurement Equation}

This section aims at deriving a batch measurement equation of the form:

$$
\mathbf{z}=\mathbf{H} \mathbf{x}+\mathbf{v}
$$

which expresses the linear relationship between the measurements/observations $\mathbf{z}$ and the states $\mathbf{x}$ made of user parameters over time. We start with the linearized measurement equations for ionosphere-error-free carrier and code phase measurements Eq. (3.1) for satellite $i$ at time $k$ written here again as

$$
\begin{aligned}
{ }^{i} \phi_{k} & =-{ }^{i} \mathrm{e}^{\mathrm{T}} \mathbf{x}_{k}+\tau_{k}+{ }^{i} \eta+{ }^{i} \mathscr{E}_{E, \phi, k}+{ }^{i} \mathscr{E}_{T, \phi, k}+{ }^{i} \mathscr{E}_{M, \phi, k}+{ }^{i} \mathscr{E}_{R, \phi, k} \\
{ }^{i} \rho_{k} & =-{ }^{i} \mathrm{e}_{k}^{\mathrm{T}} \mathbf{x}_{k}+\tau_{k}+{ }^{i} \mathscr{E}_{E, \rho, k}+{ }^{i} \mathscr{E}_{T, \rho, k}+{ }^{i} \mathscr{E}_{M, \rho, k}+{ }^{i} \mathscr{E}_{R, \rho, k}
\end{aligned} .
$$

For convenience in building the matrices for batch processing, the following vectors are defined

$$
\begin{aligned}
\mathbf{u}_{k} & =\left[\begin{array}{c}
\mathbf{x}_{k} \\
\tau_{\mathrm{GPS}, k} \\
\tau_{\mathrm{Gal}, k}
\end{array}\right] \\
{ }^{i} \mathbf{g}_{k}^{\mathrm{T}} & =\left[\begin{array}{lll}
-{ }^{i} \mathrm{e}_{k}^{\mathrm{T}} & 1 & 0
\end{array}\right] \text { if satellite } i \text { is a GPS, OneWeb, or Boeing satellite } \\
{ }^{i} \mathbf{g}_{k}^{\mathrm{T}} & =\left[\begin{array}{lll}
-{ }^{i} \mathrm{e}_{k}^{\mathrm{T}} & 0 & 1
\end{array}\right] \text { if satellite } i \text { is a Galileo satellite } \\
{ }^{i} \nu_{k, \phi} & ={ }^{i} \mathscr{E}_{E, \phi, k}+{ }^{i} \mathscr{E}_{T, \phi, k}+{ }^{i} \mathscr{E}_{M, \phi, k}+{ }^{i} \mathscr{E}_{R, \phi, k} \\
{ }^{i} \nu_{k, \rho} & ={ }^{i} \mathscr{E}_{E, \rho, k}+{ }^{i} \mathscr{E}_{T, \rho, k}+{ }^{i} \mathscr{E}_{M, \rho, k}+{ }^{i} \mathscr{E}_{R, \rho, k}
\end{aligned}
$$

Galileo employs a different time reference from GPS Time, which results in a distinct receiver clock bias $\tau_{\mathrm{Gal}, \mathrm{k}}$. In this preliminary analysis, mega-constellations are assumed to be synchronized with GPS, 
and share the same receiver clock bias $\tau_{\mathrm{GPS}, k}$. In future work, this assumption can be relaxed to where each constellation can have their own clock bias, and satellites are time-synchronized only within each constellation.

Consider measurements for both GNSS and LEO satellites from times 1 to $q$. Measurements from distinct satellites are designated by the left superscript $i$, which goes from 1 to $n$, where $n$ is the total number of satellites in view. For clarity of presentation, we consider that measurements for visible satellites are available throughout the batch period. In practice, satellites can rise and set within the batch period, and this occurrence is frequent for low Earth-orbiting (LEO) satellites. Our actual implementation accounts for satellites coming in and out of view. Further details on procedures to account for satellites rising and setting can be found in [48].

We stack measurements over time for distinct satellites $i$, which gives us the vectors below. Vectors that represent measurement stacks are denoted in bold face. We use the notation

$$
{ }^{i} \mathbf{G}=\left[\begin{array}{ccc}
{ }^{i} \mathbf{g}_{1}^{\mathrm{T}} & & 0 \\
& \ddots & \\
\mathbf{0} & & { }^{i} \mathbf{g}_{q}^{\mathrm{T}}
\end{array}\right] \quad{ }^{i} \boldsymbol{\varphi}=\left[\begin{array}{c}
{ }^{i} \phi_{1} \\
\vdots \\
{ }^{i} \phi_{q}
\end{array}\right] \quad{ }^{i} \boldsymbol{\rho}=\left[\begin{array}{c}
{ }^{i} \rho_{1} \\
\vdots \\
{ }^{i} \rho_{q}
\end{array}\right] \quad{ }^{i} \mathbf{v}_{\phi}=\left[\begin{array}{c}
{ }^{i} \nu_{1, \phi} \\
\vdots \\
{ }^{i} \nu_{q, \phi}
\end{array}\right] \quad{ }^{i} \mathbf{v}_{\rho}=\left[\begin{array}{c}
{ }^{i} \nu_{1, \rho} \\
\vdots \\
{ }^{i} \nu_{q, \rho}
\end{array}\right] \quad \mathbf{u}=\left[\begin{array}{c}
\mathbf{u}_{1} \\
\vdots \\
\mathbf{u}_{q}
\end{array}\right]
$$

The measurements get stacked with GNSS carrier phase measurements first, followed by LEO carrier phase measurements, then GNSS code measurements. The resulting stack of measurements is

$$
\left[\begin{array}{c}
{ }^{1} \boldsymbol{\varphi} \\
{ }^{2} \boldsymbol{\varphi} \\
\vdots \\
{ }^{n} \boldsymbol{\varphi} \\
{ }^{1} \boldsymbol{\rho} \\
{ }^{2} \boldsymbol{\rho} \\
\vdots \\
{ }^{G N S S} \boldsymbol{\rho}
\end{array}\right]=\left[\begin{array}{ccccc}
{ }^{1} \mathbf{G} & \mathbf{1}_{q \times 1} & 0 & & 0 \\
{ }^{2} \mathbf{G} & 0 & \mathbf{1}_{q \times 1} & & 0 \\
\vdots & \vdots & \vdots & \ddots & \vdots \\
{ }^{n} \mathbf{G} & 0 & 0 & & \mathbf{1}_{q \times 1} \\
{ }^{1} \mathbf{G} & 0 & 0 & & 0 \\
{ }^{2} \mathbf{G} & 0 & 0 & & 0 \\
\vdots & \vdots & \vdots & \ddots & \vdots \\
{ }^{n} G N S G & 0 & 0 & & 0
\end{array}\right]\left[\begin{array}{c}
\mathbf{u} \\
{ }^{1} \eta \\
{ }^{2} \eta \\
\vdots \\
{ }^{n} \eta
\end{array}\right]+\left[\begin{array}{c}
{ }^{1} \mathbf{v}_{\phi} \\
{ }^{2} \mathbf{v}_{\phi} \\
\vdots \\
{ }^{n} \mathbf{v}_{\phi} \\
{ }^{1} \mathbf{v}_{\rho} \\
{ }^{2} \mathbf{v}_{\rho} \\
\vdots \\
{ }^{n}{ }_{G N S S} \mathbf{v}_{\rho}
\end{array}\right]
$$

where the left superscript $n_{G N S S}$ distinguishes the number of visible GNSS satellites from the left su- 
perscript $n$ indicating the total number of visible satellites (including LEOs). For further simplification, the vectors and matrices from each satellite $i$, for $i=1, \cdots, n$ are stacked into the following vectors

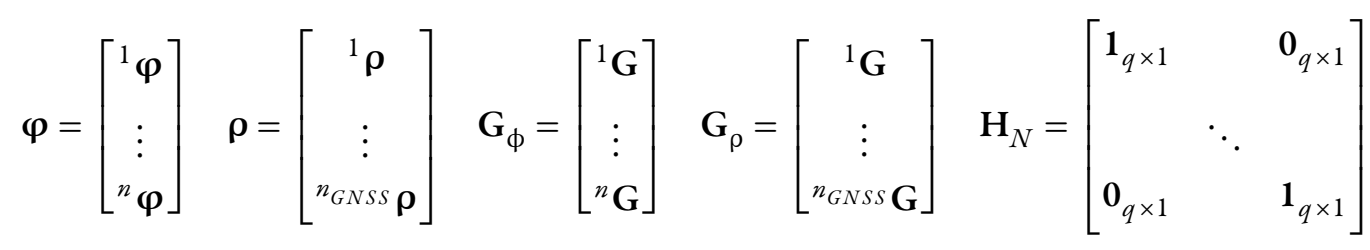

$$
\begin{aligned}
& \boldsymbol{\eta}=\left[\begin{array}{c}
{ }^{1} \eta \\
\vdots \\
{ }^{\eta} \eta
\end{array}\right] \quad \mathbf{v}_{\phi}=\left[\begin{array}{c}
{ }^{1} \mathbf{v}_{\phi} \\
\vdots \\
{ }^{n} \mathbf{v}_{\phi}
\end{array}\right] \quad \mathbf{v}_{\rho}=\left[\begin{array}{c}
{ }^{1} \mathbf{v}_{\rho} \\
\vdots \\
{ }{ }^{G N S S} \mathbf{v}_{\rho}
\end{array}\right]
\end{aligned}
$$

where we have dropped the left superscript to indicate that the non-italicized bold-faced symbols represent all the measurements. Finally, we get the batch measurement equation in the form of Eq. (4.1)

$$
\left[\begin{array}{c}
\boldsymbol{\varphi} \\
\boldsymbol{\rho}
\end{array}\right]=\left[\begin{array}{cc}
\mathbf{G}_{\phi} & \mathbf{H}_{N} \\
\mathbf{G}_{\rho} & 0
\end{array}\right]\left[\begin{array}{l}
\mathbf{u} \\
\boldsymbol{\eta}
\end{array}\right]+\left[\begin{array}{c}
\mathbf{v}_{\phi} \\
\mathbf{v}_{\rho}
\end{array}\right]
$$

where

$$
\mathbf{z}=\left[\begin{array}{c}
\boldsymbol{\rho} \\
\boldsymbol{\rho}
\end{array}\right] \quad \mathbf{H}=\left[\begin{array}{cc}
\mathrm{G}_{\phi} & \mathbf{H}_{N} \\
\mathbf{G}_{\rho} & 0
\end{array}\right] \quad \mathbf{x}=\left[\begin{array}{l}
\mathbf{u} \\
\boldsymbol{\eta}
\end{array}\right] \quad \mathbf{v}=\left[\begin{array}{c}
\mathbf{v}_{\phi} \\
\mathbf{v}_{\rho}
\end{array}\right]
$$

\subsection{Batch Measurement Error Covariance Matrix Accounting for Measure- ment Error Time Correlation}

Recall from Section 3.2 that the measurement error models for the satellite ephemeris, tropospheric, and multipath error are correlated in time for each satellite. The subtleties involved in these error models make the formulation of the measurement error covariance worth going over in detail. We start deriving the batch measurement error covariance matrix from the definition of expected value $\mathbf{V}=E\left\{\mathbf{v v}^{\mathrm{T}}\right\}$ where $E\{\cdot\}$ denotes the expected value operator. From this general definition, we will keep breaking it 
down until we arrive at the expression for the time-correlated error terms.

$$
\mathbf{V}=E\left\{\left[\begin{array}{c}
\mathbf{v}_{\phi} \\
\mathbf{v}_{\rho}
\end{array}\right]\left[\begin{array}{ll}
\mathbf{v}_{\phi}^{\mathrm{T}} & \mathbf{v}_{\rho}^{\mathrm{T}}
\end{array}\right]\right\}=\left[\begin{array}{cc}
\mathbf{v}_{\phi \phi} & \mathbf{v}_{\phi \rho} \\
\mathbf{V}_{\rho \phi} & \mathbf{v}_{\rho \rho}
\end{array}\right]
$$

The satellite ephemeris and residual tropospheric error affects code and carrier phase measurements equally, so their error covariance matrices are therefore the same. However, multipath and receiver noise error have different effects on code and carrier phase measurements. So we decompose the covariance matrix into two matrices.

$$
\mathbf{V}=\left[\begin{array}{ll}
\mathbf{V}_{\phi \phi} & \mathbf{V}_{\phi \rho} \\
\mathbf{V}_{\rho \phi} & \mathbf{V}_{\rho \rho}
\end{array}\right]=\left[\begin{array}{cc}
\mathbf{v}_{E}+\mathbf{V}_{T} & \mathbf{V}_{E}+\mathbf{V}_{T} \\
\mathbf{V}_{E}+\mathbf{V}_{T} & \mathbf{V}_{E}+\mathbf{V}_{T}
\end{array}\right]+\left[\begin{array}{cc}
\mathbf{v}_{\phi \phi, M}+\mathbf{V}_{\phi \phi, R} & 0 \\
0 & \mathbf{V}_{\rho \rho, M}+\mathbf{V}_{\rho \rho, R}
\end{array}\right]
$$

There is no time correlation for receiver noise error, so deriving $\mathbf{V}_{R}$ is straightforward and need not be included here. Equivalent derivations can be written for covariance matrices $\mathbf{V}_{E}, \mathbf{V}_{T}$, and $\mathbf{V}_{M}$ which have time correlated errors, so we take a generic approach which we denote with the subscript $\varepsilon$. Expanding $\mathbf{V}_{\varepsilon}$ we get the block diagonal matrix

$$
\mathbf{V}_{\varepsilon}=\left[\begin{array}{cccc}
{ }^{1} \mathbf{V}_{\varepsilon} & & & \mathbf{0} \\
& { }^{2} \mathbf{V}_{\varepsilon} & & \\
& & \ddots & \\
\mathbf{0} & & & { }^{n} \mathbf{V}_{\varepsilon}
\end{array}\right]
$$

which reflects that there is no error correlation between different satellites. Next, we expand one non-zero diagonal block corresponding to a single satellite $i$ which can be written as

$$
{ }^{i} \mathbf{V}_{\varepsilon}=\mathbf{E}\left\{\left[\begin{array}{ccc}
{ }^{i} \mathscr{E}_{1}{ }^{i} \mathscr{E}_{1} & \ldots & { }^{i} \mathscr{E}_{1}{ }^{i} \mathscr{E}_{q} \\
\vdots & \ddots & \vdots \\
{ }^{i} \mathscr{E}_{q}{ }^{i} \mathscr{E}_{1} & \ldots & { }^{i} \mathscr{E}_{q}{ }^{i} \mathscr{E}_{q}
\end{array}\right]\right\}
$$

where the off-diagonal terms reflect the time correlation between the errors. The generic equation for 
the covariance between errors at time $r$ and a later time $s$ can be written as

$$
\mathbf{E}\left\{{ }^{i} \mathscr{E}_{r}{ }^{i} \mathscr{E}_{s}\right\}={ }^{i} c_{r}{ }^{i} c_{s}{ }^{i} \sigma^{2} e^{-\left(\frac{t_{s}-t_{r}}{T}\right)}
$$

where ${ }^{i} c$ is the elevation-dependent coefficient, $\sigma$ is the Gauss-Markov process (GMP) standard deviation, and $T$ is the GMP correlation time constant defined in Section 3.2. Going forward, we keep a generic approach and omit the superscripts $i$ for simplicity. Given that the measurement sampling period $T_{K}$ is fixed, we define

$$
\beta=e^{-\left(\frac{T_{K}}{T}\right)}
$$

such that for satellite $i$, we can write the following:

$$
\begin{aligned}
& E\left\{\mathscr{E}_{1} \mathscr{E}_{1}\right\}=c_{1}^{2} \beta^{0} \sigma^{2}=c_{1}^{2} \sigma^{2} \\
& E\left\{\mathscr{E}_{1} \mathscr{E}_{2}\right\}=c_{1} c_{2} \beta^{1} \sigma^{2}=E\left\{\mathscr{E}_{2} \mathscr{E}_{1}\right\} \\
& E\left\{\mathscr{E}_{1} \mathscr{E}_{3}\right\}=c_{1} c_{3} \beta^{2} \sigma^{2}=E\left\{\mathscr{E}_{3} \mathscr{E}_{1}\right\} \\
& \vdots \quad \vdots \\
& E\left\{\mathscr{E}_{1} \mathscr{E}_{q}\right\}=c_{1} c_{q} \beta^{q-1} \sigma^{2}
\end{aligned}
$$

where we make no reference to the satellite ID and error source. Equation (4.12) can then be written in the generic form

$$
{ }^{i} \mathbf{V}_{\varepsilon}=\left[\begin{array}{ccccc}
c_{1}^{2} \sigma^{2} & c_{1} c_{2} \beta \sigma^{2} & c_{1} c_{3} \beta^{2} \sigma^{2} & \ldots & c_{1} c_{q} \beta q-1 \\
c_{1} c_{2} \beta \sigma^{2} & c_{2}^{2} \sigma^{2} & c_{2} c_{3} \beta \sigma^{2} & & \\
c_{1} c_{3} \beta^{2} \sigma^{2} & c_{2} c_{3} \beta \sigma^{2} & c_{3}^{2} \sigma^{2} & & \vdots \\
\vdots & & & \ddots & \\
c_{1} c_{q} \beta q-1 & & \ldots & & c_{q}^{2} \sigma^{2}
\end{array}\right] .
$$

For algorithm coding convenience, equation (4.15) can be further decomposed into the following three 
terms

$$
{ }^{i} \mathbf{V}_{\varepsilon}=\sigma^{2}\left[\begin{array}{ccccc}
c_{1}^{2} & c_{1} c_{2} & c_{1} c_{3} & \ldots & c_{1} c_{q} \\
c_{1} c_{2} & c_{2}^{2} & c_{2} c_{3} & & \\
c_{1} c_{3} & c_{2} c_{3} & c_{3}^{2} & & \vdots \\
\vdots & & & \ddots & \\
c_{1} c_{q} & & \ldots & & c_{q}^{2}
\end{array}\right] \circ\left[\begin{array}{ccccc}
\beta^{0} & \beta^{1} & \beta^{2} & \ldots & \beta^{q-1} \\
\beta^{1} & \beta^{0} & \beta^{1} & & \\
\beta^{2} & \beta^{1} & \beta^{0} & & \vdots \\
\vdots & & & \ddots & \\
\beta^{q-1} & & \ldots & & \beta^{0}
\end{array}\right]
$$

where $\circ$ indicates element-by-element multiplication of matrices. We make the following definitions

$$
\mathbf{c}=\left[\begin{array}{c}
c_{1} \\
c_{2} \\
c_{3} \\
\vdots \\
c_{q}
\end{array}\right] \quad \boldsymbol{\beta}=\left[\begin{array}{ccccc}
0 & 1 & 2 & \ldots & q-1 \\
1 & 0 & 1 & & \\
2 & 1 & 0 & & \vdots \\
\vdots & & & \ddots & \\
q-1 & & \ldots & & 0
\end{array}\right]
$$

where $\mathbf{c}$ is the vector of elevation-dependent coefficients stacked at all times for satellite $i$. These definitions give the compact expression for the measurement error covariance of satellite $i$ as

$$
{ }^{i} \mathbf{V}_{\varepsilon}=\sigma^{2} \operatorname{cc}^{\mathrm{T}} \circ \exp \circ\left(-\frac{T_{K}}{T} \boldsymbol{\beta}\right)
$$

where exp $\circ$ represents the element-wise exponential function applied to matrix $\left(-\frac{T_{K}}{T} \boldsymbol{\beta}\right)$. We can now specify expressions for covariance matrices ${ }^{i} \mathbf{V}_{E},{ }^{i} \mathbf{V}_{T}$, and ${ }^{i} \mathbf{V}_{M}$. The expression for ${ }^{i} \mathbf{V}_{R}$ is also included for completeness.

$$
\begin{aligned}
{ }^{i} \mathbf{V}_{E} & =\sigma_{E}^{2} \exp \left(-\frac{T_{K}}{T_{E}} \boldsymbol{\beta}\right) \\
{ }^{i} \mathbf{V}_{T} & =\sigma_{T}^{2}{ }^{i} \mathbf{c}_{T}{ }^{i} \mathbf{c}_{T}^{\mathrm{T}} \circ \exp \left(-\frac{T_{K}}{T_{T}} \boldsymbol{\beta}\right) \\
{ }^{i} \mathbf{V}_{M} & =\sigma_{M}^{2}{ }^{i} \mathbf{c}_{M}{ }^{i} \mathbf{c}_{M}^{\mathrm{T}} \circ \exp \left(-\frac{T_{K}}{T_{M}} \boldsymbol{\beta}\right) \\
{ }^{i} \mathbf{V}_{R} & =\operatorname{diag}\left({ }^{i} \sigma_{R, 1}^{2}, \quad{ }^{i} \sigma_{R, 2}^{2}, \quad \ldots,{ }^{i} \sigma_{R, q}^{2}\right)
\end{aligned}
$$




\subsection{Batch Estimator}

Given the linear observation state relationship expressed in (4.1), the weighted least squares estimate $\hat{\mathbf{x}}$ that minimizes the sum of squares of the weighted observation errors is given as

$$
\hat{\mathbf{x}}=\left(\mathbf{H}^{\mathrm{T}} \mathbf{W} \mathbf{H}\right)^{-1} \mathbf{H}^{\mathrm{T}} \mathbf{W} \mathbf{z}
$$

where the weighting matrix $\mathbf{W}=\mathbf{V}^{-1}$, the inverse of the batch measurement error covariance matrix $\mathbf{V}$. Equation (4.20) can also be expressed as

$$
\hat{\mathbf{x}}=\mathbf{P} \mathbf{H}^{\mathrm{T}} \mathbf{W} \mathbf{z}
$$

where

$$
\mathbf{P}=\left(\mathbf{H}^{\mathrm{T}} \mathbf{W} \mathbf{H}\right)^{-1}
$$

is the covariance matrix of the estimation errors associated with $\hat{\mathbf{x}}$. The covariance matrix $\mathbf{P}$ describes the uncertainty in the user position and receiver clock bias at all times of measurements 1 through $q$, and the uncertainty in the estimated constant cycle ambiguities. The final position covariance at time $q$ in the North-East-Down (NED) direction, written as $\mathbf{P}_{\mathrm{NED}, q}$, is a $3 \times 3$ matrix that is fully populated. For ground vehicle applications, the horizontal position uncertainty is most important. So in this work, we are primarily concerned with the North and East directions, and will be dealing with the $2 \times 2$ current time horizontal position covariance $\mathbf{P}_{\mathrm{NE}, q}$. This $2 \mathrm{D}$ covariance matrix, can be represented as a covariance ellipse. We determine the maximum horizontal positioning uncertainty by finding the maximum eigenvalue of $\mathbf{P}_{\mathrm{NE}, q}$, computed as

$$
\sigma_{\max }^{2}=\max \left(\operatorname{eig}\left(\mathbf{P}_{\mathrm{NE}, q}\right)\right)
$$




\subsection{Protection Level Derivation}

In this section, we use the navigation metrics of accuracy, integrity, continuity, and availability to develop a framework for evaluating the global fault-free availability of mega-constellation-augmented GNSS (GNSS-MC). First, we give the following definitions [8]:

Horizontal alert limit (HAL) is the radius of a circle centered at the true position, that circumscribes the region required to contain the estimated horizontal position with the required probability (i.e. integrity risk requirement).

Horizontal protection level (HPL) is the radius of a circle centered at the true position, that circumscribes the region assured to contain the estimated horizontal position with quantifiable risk.

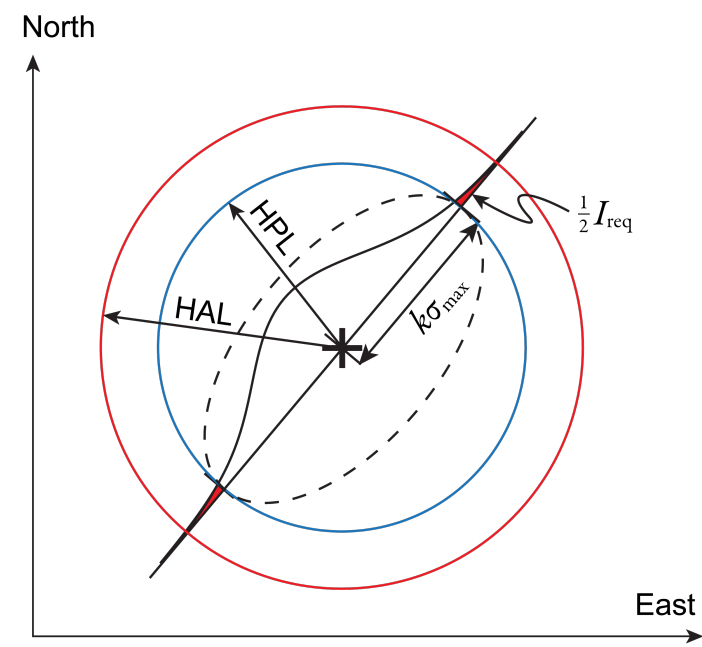

Figure 4.1: Simplified Illustration Depicting the Horizontal Protection Level and Horizontal Alert Limit.

Figure 4.1 depicts HPL and HAL visually. The crosshair represents the true horizontal position in the North and East direction. The probability density function for the error is depicted in the direction of the maximum horizontal position uncertainty. The dashed ellipse represents a scaled horizontal position covariance ellipse.

In this fault-free availability analysis, the GNSS-MC system is required to meet an example integrity risk requirement of $I_{\text {req }}=10^{-5}$. This means that the probability that the horizontal positioning error exceeds HPL is less than or equal to $10^{-5}$ or $P\left(\left|\mathscr{E}_{H}\right|>\mathrm{HPL}, H_{0}\right) \leq I_{\text {req }}$, where $H_{0}$ indicates that this is fault-free. For this integrity risk requirement, the fault-free horizontal protection level $\left(\mathrm{HPL}_{0}\right)$ is computed as

$$
\mathrm{HPL}_{0}=k \sigma_{\max }
$$


where

$$
k=Q^{-1}\left(\frac{I_{\text {req }}}{2}\right)
$$

and $\sigma_{\max }$ is given by Eq. (4.23). The $Q$-function is the tail distribution function of the standard normal distribution, and $Q^{-1}$ is the inverse $Q$-function. In other words, $I_{\text {req }} / 2$ is the probability that a standard normal variable takes a value larger than $k$.

Significant work and testing goes into the definition of a horizontal alert limit, and it is applicationspecific (e.g. different between aircraft and automotive applications). In this preliminary work, we take the horizontal alert limit to be $1.5 \mathrm{~m}$, and the availability criterion is $\mathrm{HPL}_{0}<\mathrm{HAL}=1.5 \mathrm{~m}$. This example value for HAL was chosen to generate results in Section 5.3.1, and will be refined in future work for specific applications. 


\section{Chapter 5}

\section{Performance Analysis}

In this chapter we present results obtained using the positioning algorithm derived in Chapter 4 . We consider simulations of the horizontal positioning accuracy obtained using GNSS only, GNSS-OneWeb, and GNSS-Boeing in the context of a nominal case, and variations thereof. A sensitivity analysis is performed to evaluate the effects of varying elevation mask, batch period, measurement sampling rate, and orbit ephemeris error.

We begin this chapter with a discussion of the covariance analysis implementation as it applies to the nominal case. This implementation forms the basis for all subsequent performance analysis which includes a sensitivity analysis for a single reference location, and a global fault-free availability analysis. This section aims to answer the questions posed in the introduction: Can mega-constellations, when combined with GNSS, contribute to achieving safety-critical integrity risk requirements? Can they do so in urban canyons where low-elevation satellite signals are blocked?

\subsection{Covariance Analysis Implementation}

In this section, we describe the implementation of a covariance analysis as it applies to the nominal case. This example uses OneWeb-augmented GNSS and a 40-degree elevation mask. A 10-minute batch is performed. Within the batch period, measurements are sampled for the estimator every 1 minute. We 
simulate such batches repeatedly at regular 1 minute intervals over 24 hours from a reference location in Tucson, Arizona $\left(32.2369278^{\circ} \mathrm{N}, 110.951933^{\circ} \mathrm{W}, 752 \mathrm{~m}\right.$ altitude).It is important to note that the measurement sampling rate and batch period are chosen so that we are taking measurements frequent enough to average out noise, and the batch period is long enough to take advantage of satellite motion. Based on each 10-min batch, the final position covariance is stored for analysis.
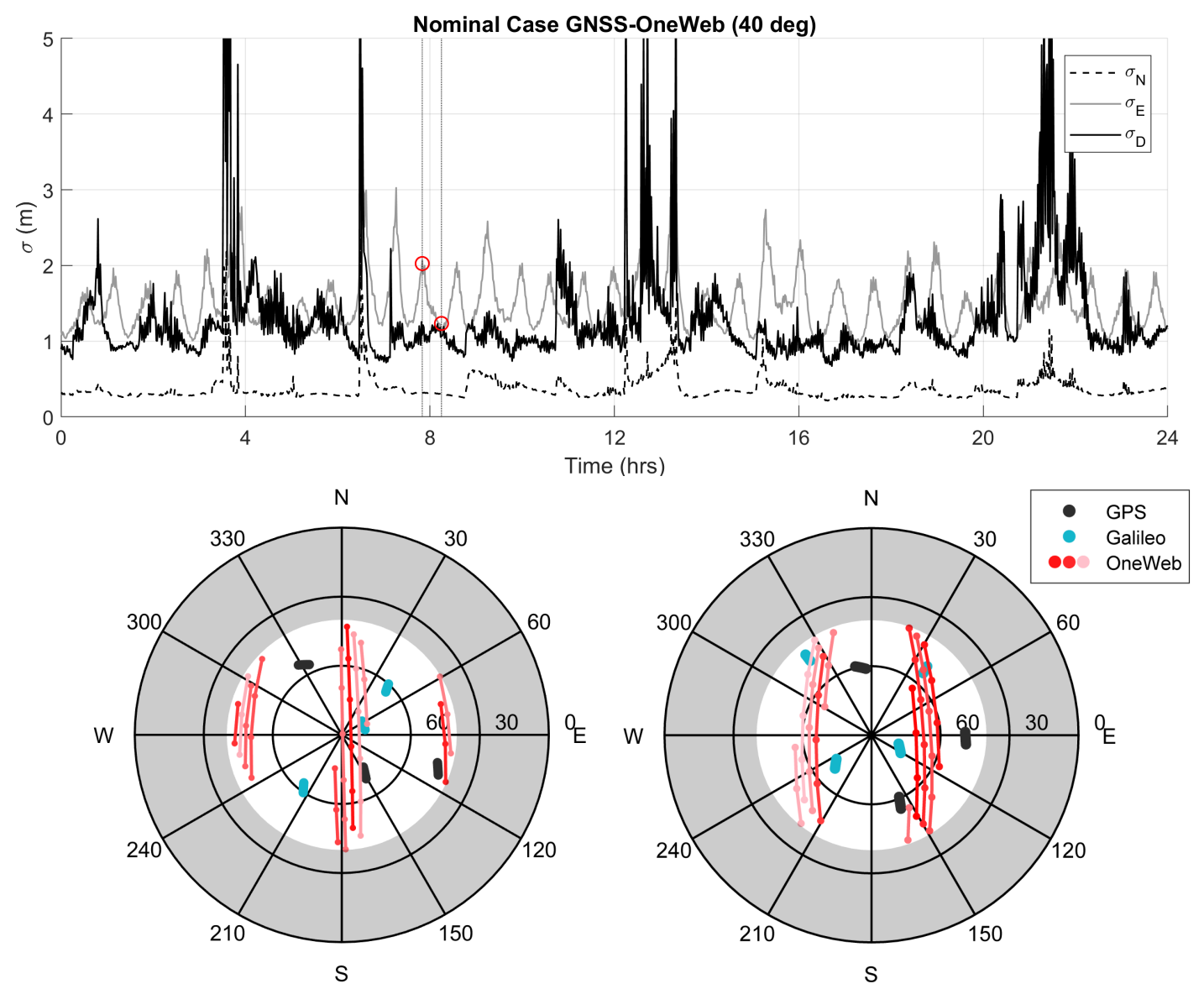

Figure 5.1: Horizontal Positioning Performance for OneWeb-augmented GNSS Using a $40^{\circ}$ Elevation Mask in Tucson, Arizona. The top plot shows the positioning error standard deviation in the North, East, and Down directions during the 24-hour simulation. The two azimuth-elevation (AzEl) plots are for two time instances indicated by red circles and vertical dotted lines on the top plot. The left AzEl plot is for the batch at 7.83-hr where $\sigma_{E}$ is at a relative maximum. The right AzEl plot is for the batch at $8.25-\mathrm{hr}$ where $\sigma_{E}$ is at a relative minimum.

Figure 5.1 shows the positioning performance using GNSS-OneWeb for a 24-hour simulation for a user in Tucson, Arizona. On the top plot we look at the individual standard deviations in the North, East, and Down direction for the entire day for an elevation mask of $40^{\circ}$. 
The OneWeb constellation uses near polar orbits, which means that over the 10-minute batch period while the GNSS satellites appear static for a user on Earth, OneWeb satellites cross the entire sky in the North-South direction. As a result, the uncertainty in the North direction is lowest. At a high elevation mask of 40 degrees, the positioning error in the Down direction has the largest peaks. The positioning error in the East direction suffers from the North-South directionality of satellite motion, and but is otherwise bounded between 1 and 2-3 meters. This peculiar behavior of $\sigma_{E}$ can be explained with the help of the two azimuth-elevation (AzEl) plots on the bottom of Figure 5.1. The fluctuation of $\sigma_{E}$ is due to the fact that the Earth rotates while the LEO satellites move in quasi-stationary orbital planes. The reference location on Earth moves East-to-West with respect to the relatively stationary near-polar OneWeb orbits. Over time, the reference location gets placed from a location where the OneWeb satellites traverse directly overhead, to another location where it is in between two OneWeb orbital planes. Peaks in $\sigma_{E}$ occur when the user is directly below an orbital plane, and most of the satellites are concentrated in the center of the sky. The valleys in $\sigma_{E}$ occur when the user is in between two orbital planes, and the satellites are distributed on either side. Reference [36] gives a detailed explanation of a similar observation for navigation using GPS and Iridium (a 66-satellite LEO communications constellation with near polar orbits).

\subsection{Performance Sensitivity Analysis}

The objective of this section is to identify sensitive parameters that are key to leveraging future communication mega-constellations in safety-critical navigation applications. To achieve this, we do a detailed analysis at a single user location. The subsequent sections comprise a local performance analysis investigating the effects of elevation mask, batch period, measurement sampling rate, and ephemeris error on the positioning performance of mega-constellation-augmented GNSS. 


\subsubsection{Performance Sensitivity to Elevation Mask}

We are interested in quantifying the navigation performance of the combined system in both open sky and urban canyon environments. Urban canyon environments have views of the sky that can be partially obscured by tall buildings, bridges, trees, and other obstacles. An extreme example of this is found in Figure 5.2 [49], which depicts a panoramic picture of the sky above a car-mounted antenna in the cities of Tokyo. Here we see that the visible patch of sky has a complex shape. In this preliminary analysis, we simulate this urban canyon formed by the buildings by applying an elevation mask.

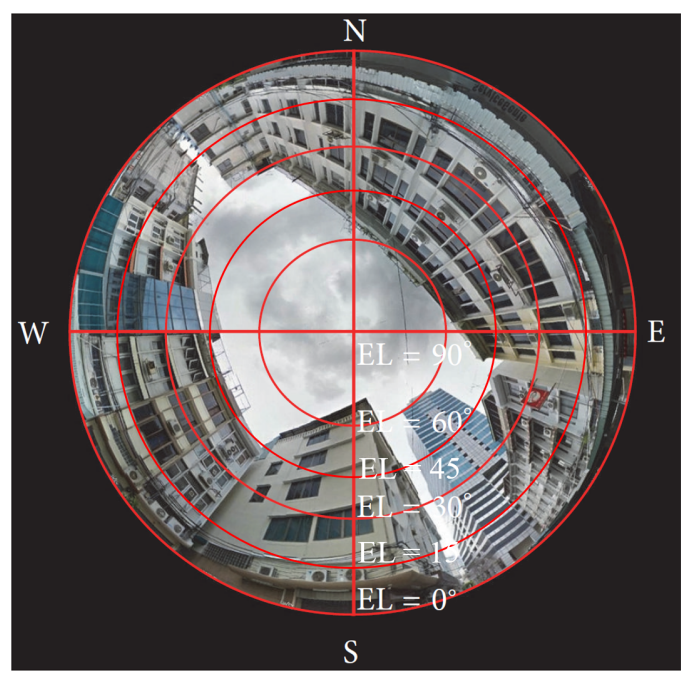

Figure 5.2: Obstructed Sky Above a CarMounted Antenna courtesy of [49]. The concentric circles each represent an elevation, and the straight lines represent azimuths.

An elevation mask restricts a user's field of view to a cone with the point at the user location, and the open end pointing to the sky. The field of view gets smaller as the elevation mask is increased. Moreover, the annular area from the horizon all the way to a certain elevation mask is considered obstructed, and satellite signals are blocked below this elevation mask.

We implement a batch covariance analysis for the range of elevation masks 5 to $60^{\circ}$ at Tucson, Arizona. At each elevation mask, 10-minute batches are simulated every minute over 24 hours. The maximum and minimum eigenvalue of the batch's current time horizontal position covariance for each 10-minute batch is stored. Finally, the maximum and minimum value among these eigenvalues for the 24-hour simulation period is plotted against the corresponding elevation mask.

The performance using GNSS only is shown on the top plot of Figure 5.3. Here, the thick black line represents the square root of the maximum eigenvalue $\left(\sigma_{\max }\right)$ of $\mathbf{P}_{\mathrm{NE}, q}$ (current time position covariance matrix for the North and East direction, see Eq. 4.23), while the thin black line represents the square 


\section{MEGA-CONSTELLATION-AUGMENTED GNSS PERFORMANCE ANALYSIS}

root of the minimum eigenvalue $\left(\sigma_{\min }\right)$. These correspond to the maximum and minimum horizontal position uncertainty for each 24-hour simulation period at every elevation mask. The bottom plot of Figure 5.3 shows the maximum and minimum number of visible satellites for the 24 hour simulation period at each elevation mask.
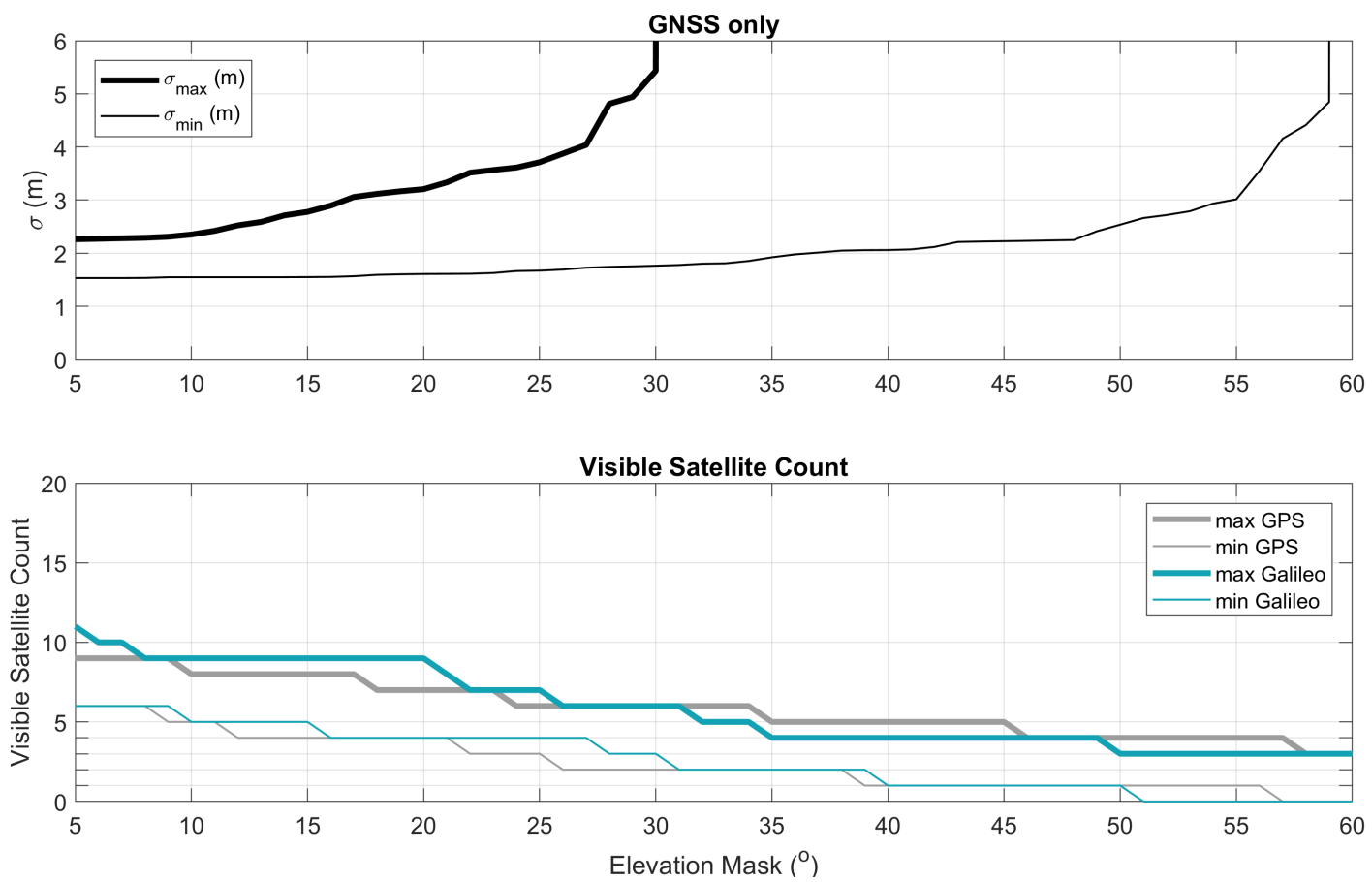

Figure 5.3: Elevation Sensitivity Performance Using GNSS Only in Tucson, Arizona. In the top plot, maximum and minimum horizontal positioning uncertainties over 24 hours are plotted for each elevation mask, for a user in Tucson, Arizona. The bottom plot shows the maximum and minimum number of visible satellites for each constellation over 24 hours for each elevation mask.

As the elevation mask increases, both $\sigma_{\max }$ and $\sigma_{\min }$ lines increase, while the number of visible satellites decreases. The $\sigma_{\max }$ data point at $30^{\circ}$ reaches about $5.5 \mathrm{~m}$, and coincides with the maximum elevation mask for which the GNSS only case is usable i.e. for which $>2$ satellites are always visible. Past $30^{\circ}$, the horizontal positioning error uncertainty can exceed $6 \mathrm{~m}$. If we can use measurements from OneWeb satellites, we expect the $\sigma_{\max }$ line to peak at an elevation mask higher than $30^{\circ}$, as we will see in the next figure. 

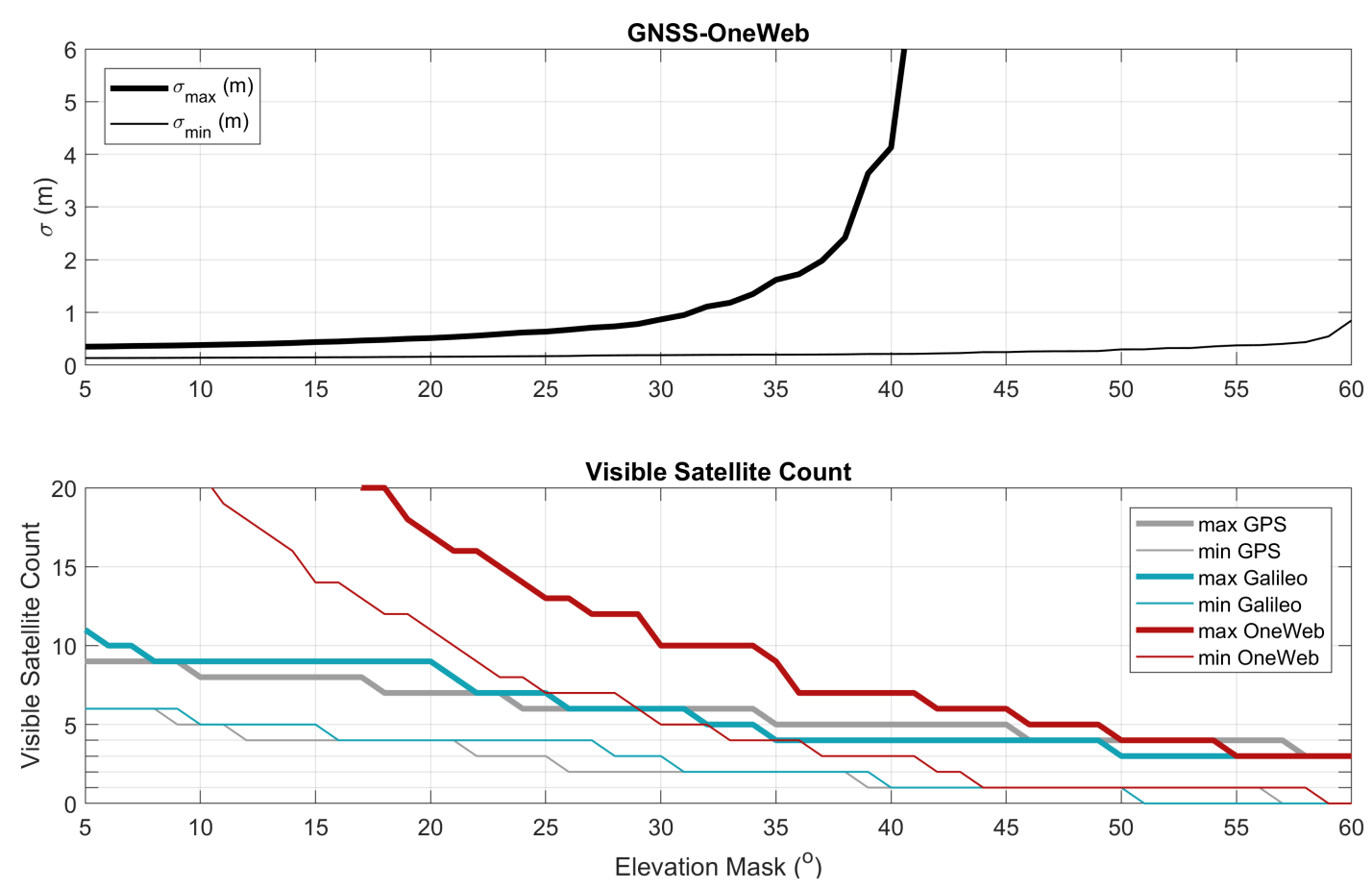

Figure 5.4: Elevation Sensitivity Performance Using GNSS-OneWeb in Tucson, Arizona. In the top plot, maximum and minimum horizontal positioning uncertainties over 24 hours are plotted for each elevation mask, for a user in Tucson, Arizona. The bottom plot shows the maximum and minimum number of visible satellites for each constellation over 24 hours for each elevation mask.

In Figure 5.4 we recognize similar general trends as with the GNSS only case in Figure 5.3. There are additional visible satellites as indicated by the red lines on the bottom plot of Figure 5.4. With these additional measurements from OneWeb, the worst case scenario (thick black line) stays under $5 \mathrm{~m}$ up to an elevation mask of $40^{\circ}$, and the best case scenario (thin black line) not only stays observable, but also remains well below $1 \mathrm{~m}$ for the range of elevation masks.

The addition of OneWeb measurements decreases the uncertainty in the final horizontal position for the full range of elevation masks. If we replace OneWeb with the Boeing constellation, we significantly increase the number of non-GNSS measurements. This should have the effect of decreasing the overall $\sigma_{\max }$ and $\sigma_{\min }$ values, and also cause the $\sigma_{\max }$ line to peak at an elevation mask higher than $40^{\circ}$. These are indeed the trends as evidenced by the next figure. 

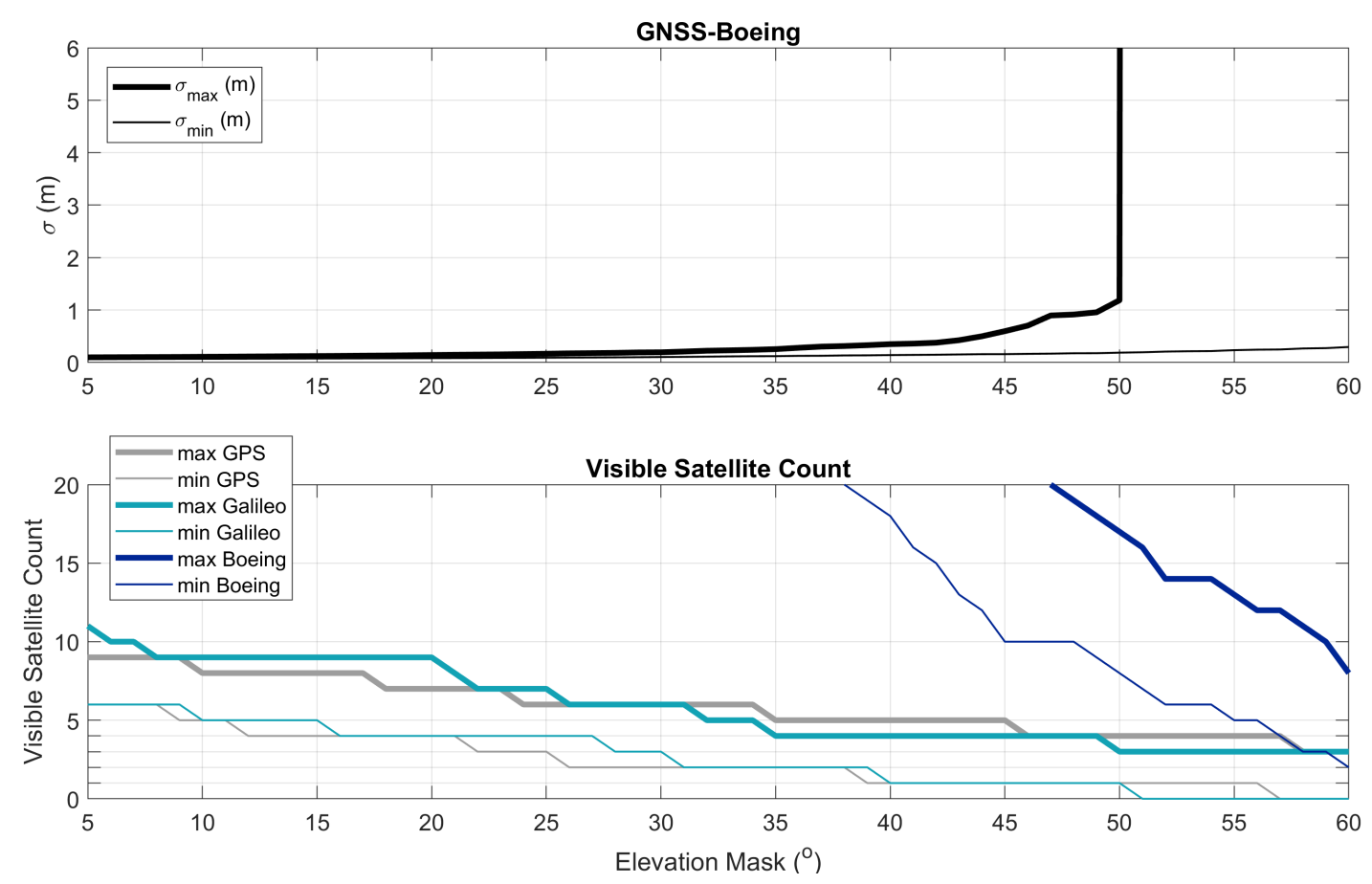

Figure 5.5: Elevation Sensitivity Performance Using GNSS-Boeing in Tucson, Arizona. In the top plot, maximum and minimum horizontal positioning uncertainties over 24 hours are plotted for each elevation mask. The bottom plot shows the maximum and minimum number of visible satellites for each constellation over 24 hours for each elevation mask.

The elevation performance sensitivity for Boeing-augmented GNSS is presented in Figure 5.5. Again, we observe the same general trends as with the previous two figures. At this scale, the best case and worst case horizontal position uncertainties are similar at elevation masks less than $35^{\circ}$. On the bottom plot of Figure 5.5 we see that the number of visible Boeing satellites start outside of the plot axes, well above 20 visible satellites. The large number of additional measurements, and the variety of the Boeing orbits (there are $45^{\circ}$ - and $55^{\circ}$-inclined orbits, and a set of near polar orbits) results in significantly lower final horizontal position uncertainties, and keeps the maximum horizontal positioning uncertainty below $1.5 \mathrm{~m}$ up to an elevation mask of $50^{\circ}$. Past $50^{\circ}$, the maximum positioning uncertainty does not grow slowly, and instead spikes up. Although this is a significant improvement over the GNSS only case, the spike indicates that we have reached a limit. The visible patch of sky is simply too small and the few ranging signals that can get through are too clustered to yield a good position estimate. 
In this section, we learned that the horizontal positioning performance when using GNSS only, GNSSOneWeb, and GNSS-Boeing drops at elevation masks greater than 30, 40, and 50 degrees respectively. Based on these results, we specify an elevation mask of $40^{\circ}$ for the nominal case that will be used in the subsequent sensitivity analysis.

\subsubsection{Performance Sensitivity to Batch Period}

The next sensitivity parameter of interest is the batch period $\left(t_{\text {batch }}\right)$. The nominal case uses a 10-minute batch period. In this analysis we look at the effect of shortening and extending the batch period on the final horizontal positioning uncertainty. The same user location is used in Tucson, Arizona. During the 24-hour simulation period, a batch covariance analysis is simulated every minute for a range of batch periods and for a fixed elevation mask of 40 degrees. We expect a decrease in positioning uncertainty as the batch period lengthens. Conversely, we expect an increase in uncertainty as the batch period is shortened. We investigate the effect of using a batch period of 5 to 25 minutes at 5 -minute intervals.

The batch period sensitivity results using GNSS only, GNSS-OneWeb, and GNSS-Boeing are shown in Figure 5.6. The results for each batch period is distinguished by the different colors. Similar to previous figures, we only plot the square root of the maximum eigenvalue of the final covariance in the horizontal direction as given by Eq. (4.23). We see the best performance for $t_{\text {batch }}=25 \mathrm{~min}$ in dark blue, and the worst performance for $t_{\text {batch }}=5 \mathrm{~min}$ in dark red. The visible satellite count over 24 hours is shown on the bottommost plot of Figure 5.6. 

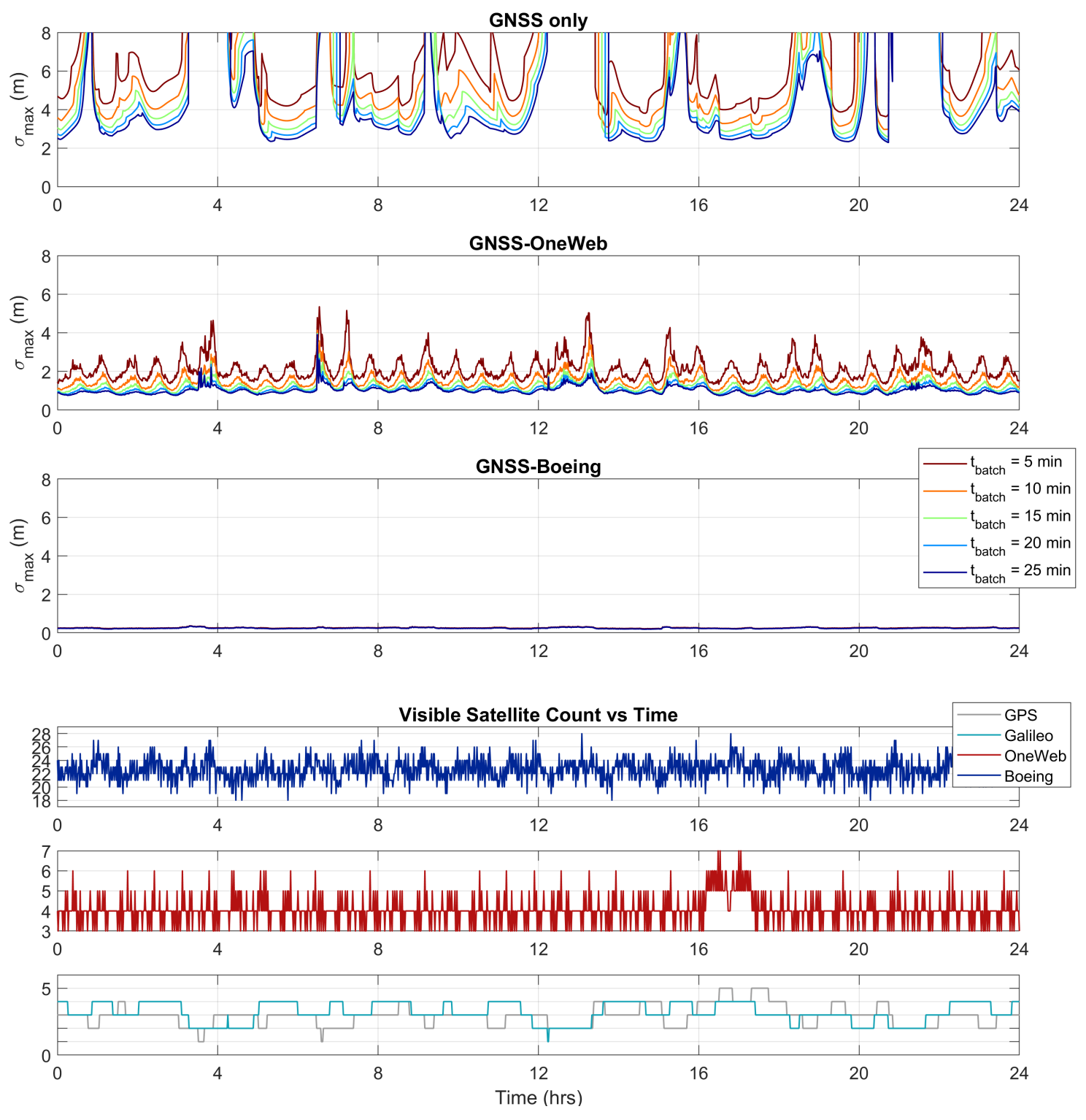

Figure 5.6: Batch Period Sensitivity Analysis Using a $40^{\circ}$ Elevation Mask in Tucson, Arizona. The top three plots show the horizontal positioning performance over time using GNSS only, GNSS-OneWeb, and GNSS-Boeing for a range of batch periods. The bottom three plots show the number of visible satellites for GNSS, OneWeb, and Boeing over 24 hours.

In the GNSS only case, increasing the batch period decreases $\sigma_{\max }$ for time periods where maximum uncertainty is under 8 meters. The spikes indicate a very large uncertainty in the horizontal user position. At these times, GNSS measurements alone perform poorly for a 40-degree elevation mask. The spikes 
coincide with times when there are at most two GPS satellites or at most two Galileo satellites. This deficiency in measurements is solved by the addition of 720 OneWeb satellites as seen on the second plot. In the GNSS-OneWeb case, $\sigma_{\max }$ stays below five meters. We still see the characteristic peaks and valleys of the horizontal positioning uncertainty as explained back in section 5.1 for Figure 5.1. The plot reveals that increasing the batch period from 5 minutes to 25 minutes, yields $0.5-2$ meter decrease in $\sigma_{\max }$. The elevation mask plays a role. It is important to realize that satellites appear to move faster near the horizon, and slower near zenith. Given the 40-degree elevation mask, the satellite motion is limited to a narrow patch of sky. Next, we look at the positioning performance using GNSS-Boeing. At this scale, changing the batch period has little effect to the consistent sub-meter $\sigma_{\max }$ values achieved using Boeing-augmented-GNSS. The whopping 20-25 additional measurements from Boeing, ensures sub-meter horizontal positioning uncertainty for Tucson over the entire 24-hour period.

\subsubsection{Performance Sensitivity to Measurement Sampling Rate}

The nominal case uses measurements sampled every minute within a 10-minute batch period. In this sensitivity analysis, we look at the effect of taking measurements more frequently and less frequently than every minute. We look specifically at measurement sampling periods of 30,60, 90, and 120 seconds for positioning using GNSS only, GNSS-OneWeb, and GNSS-Boeing. The results for all three configurations are shown in Figure 5.7.

First, we look at the results for the GNSS only case. The changes in measurement sampling rate have little effect when using GNSS only. This is caused by the fact that GNSS satellites have half-day orbital periods, and have very little apparent motion in the sky within the 10-minute batches. Taking measurements more frequently gives us "in-between" measurements that do not offer additional information. 

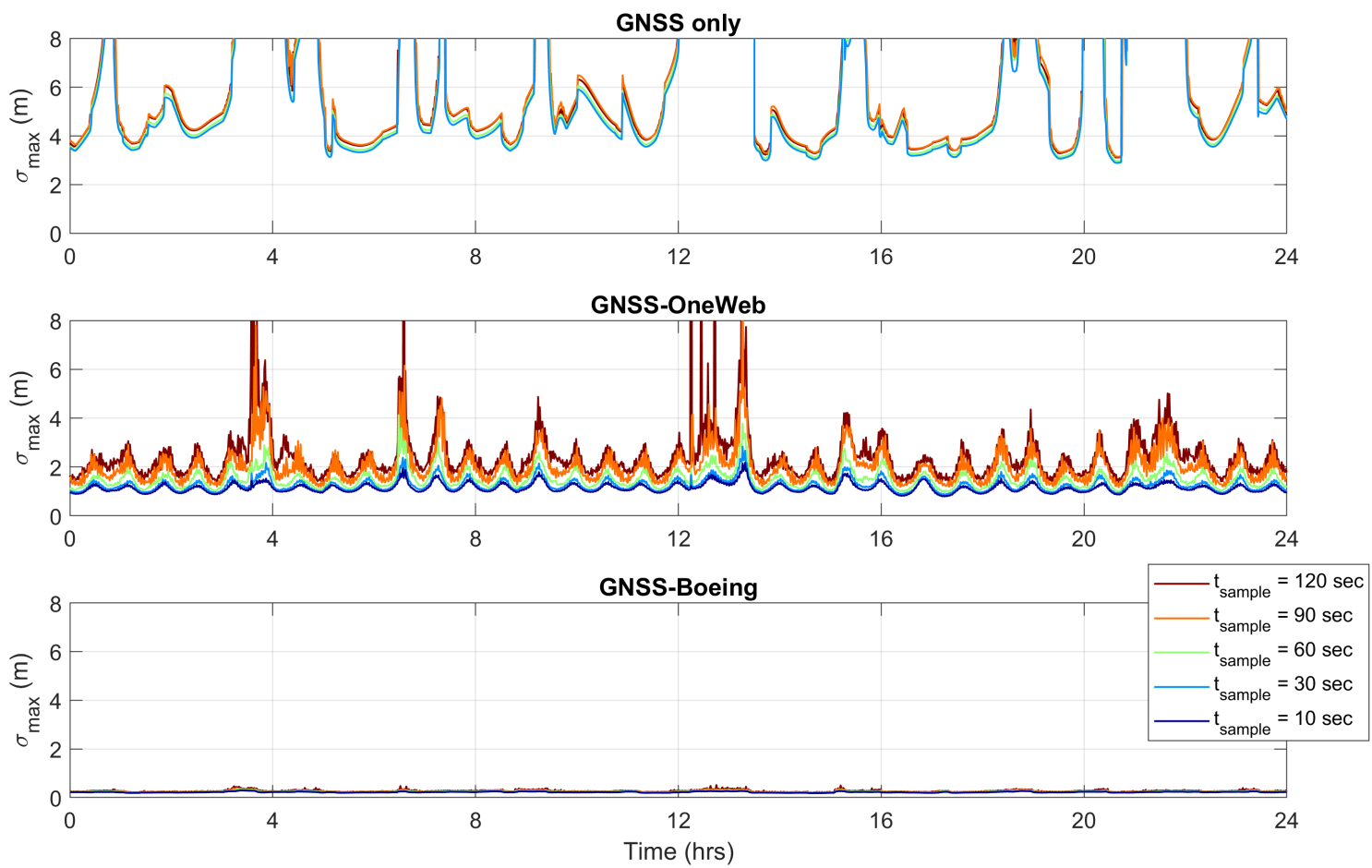

Figure 5.7: Measurement Sampling Rate Sensitivity Analysis Using a 40 Elevation Mask in Tucson, Arizona. The three plots show the horizontal positioning performance over time using GNSS only, GNSS-OneWeb, and GNSS-Boeing for a range of measurement sampling periods.

The effect of changing the measurement sampling rate is more appreciable when using GNSS-OneWeb. OneWeb satellites have an orbital period of about 100 minutes, and there is significant satellite motion provided by the OneWeb constellation within the 10-minute batch period. As a result, the horizontal positioning performance improves as the measurement sampling period gets smaller. At the measurement sampling periods of 90 and 120 seconds, we get peaks at the 4-hour mark, as well as at the 7-, 12-, and 13-hour mark.

Next, we look at the results for GNSS-Boeing on the third plot of Figure 5.7. Similar to Figure 5.6, the different sampling rates have little effect to the consistent sub-meter horizontal positioning performance when using GNSS-Boeing. Here however, the sampling rates of 120 and 90 seconds yielded noisier plots. The effect of taking measurements less frequently on the GNSS-Boeing case, is that we see more fluctuation in $\sigma_{\max }$. 


\section{MEGA-CONSTELLATION-AUGMENTED GNSS PERFORMANCE ANALYSIS}

\subsubsection{Performance Sensitivity to Satellite Orbit and Clock Error}

Back in section 2.5, we looked at measurement error sources and proceeded to model them in section 3.2. At this point in the analysis, the most unpredictable among these error sources is the satellite clock and orbit ephemeris for mega-constellations. The ephemeris error is highly dependent on the orbit determination process, update rate, space weather, and density of ground monitoring stations among others. This section will focus on the effects of the first one: the orbit determination process. We look at the effect of the orbit determination process by varying the Gauss Markov Process (GMP) standard deviation $\left(\sigma_{E, L E O}\right)$ of the satellite ephemeris error model for the mega-constellations.
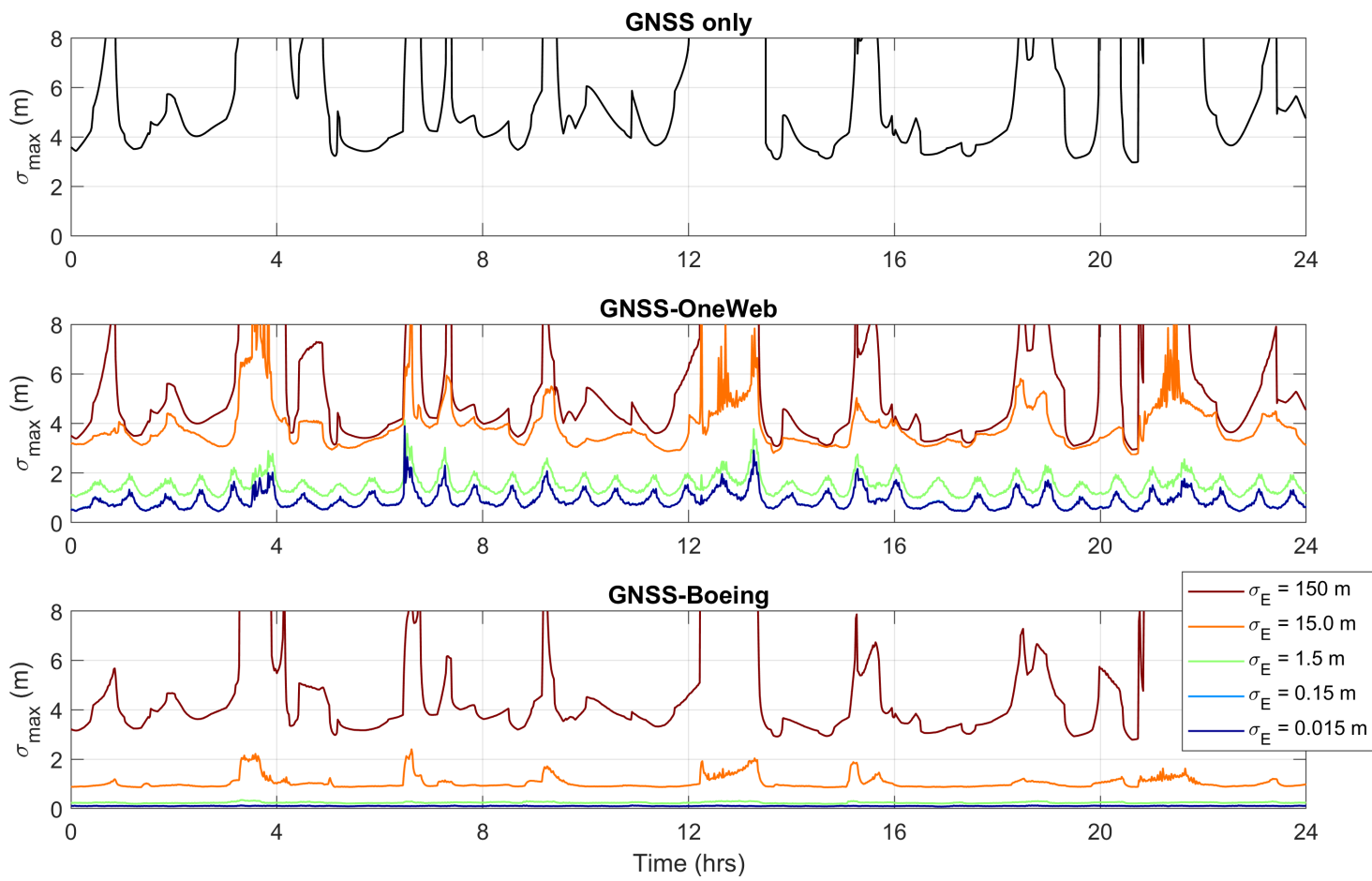

Figure 5.8: Ephemeris Error Sensitivity Analysis Using a $40^{\circ}$ Elevation Mask in Tucson, Arizona. The top three figures show the horizontal positioning performance over time using GNSS only, GNSS-OneWeb, and GNSS-Boeing for a range of LEO ephemeris error GMP standard deviations.

The positioning performance using GNSS-OneWeb and GNSS-Boeing are plotted in Figure 5.8. The nominal case using GNSS only is also included as a baseline (GNSS error parameters are kept nominal). We consider the following values for the ephemeris error GMP standard deviation: $0.015 \mathrm{~m}, 0.15 \mathrm{~m}$, $1.5 \mathrm{~m}$ (nominal case), $15 \mathrm{~m}$, and $150 \mathrm{~m}$. In the GNSS-OneWeb case, the $\sigma_{\max }$ plot for $0.015 \mathrm{~m}$ and $0.15 \mathrm{~m}$ 
are on top of each other, and provide the best horizontal positioning performance. At GMP standard deviation values of $15 \mathrm{~m}$ and $150 \mathrm{~m}$, the horizontal positioning uncertainty using GNSS-OneWeb exceeds $5 \mathrm{~m}$ for certain time periods. We look at the large GMP standard deviation value of $150 \mathrm{~m}$ to see if we can match the positioning performance using GNSS only. This is to show how much leeway we have on the mega-constellation orbit determination processes. In the GNSS-Boeing case, the positioning performance stays well below 5 meters even at a GMP standard deviation of $15 \mathrm{~m}$. This attests to the fact that the 20-25 additional measurements from Boeing provides a decent estimate of the user position, even when the uncertainty on the satellite ephemeris is $15 \mathrm{~m}$.

Considering both the 720-satellite OneWeb constellation, and 2956-satellite Boeing constellation allowed us to investigate the margin of error tolerable for satellite orbit and clock. This shows that increasing the number of satellites in view, relaxes the accuracy requirement on the orbit determination process for mega-constellations.

\subsection{Global Performance Analysis}

\subsubsection{Fault-Free Availability and Coverage Analysis}

We consider the nominal case discussed in section 5.1 and implement an equivalent covariance analysis, but this time for a grid of locations on Earth. This grid is created by dividing the globe into equallyspaced latitudes and longitudes. At each grid point, we assess the fraction of time over 24 hours that $\mathrm{HPL}_{0}$ is less than HAL. This fraction of time where $\mathrm{HPL}_{0}<\mathrm{HAL}$ corresponds to the fraction of time that the navigation system meets the example integrity requirements for safe operation, which was specified in Section 4.4. We refer to this time fraction as fault-free availability. The fault-free availability is a number between $0 \%$ and $100 \%$. For this analysis, 10-min batches are simulated at regular 10-min intervals over 24 hours (instead of the regular 1-min intervals used in the local sensitivity analysis). 
We implement separate fault-free availability analyses using GNSS only, GNSS-OneWeb, and GNSSBoeing for a horizontal alert limit of 1.5 meters in two scenarios: (1) open sky with a 5 -degree elevation mask, and (2) urban canyon with a 40-degree elevation mask. We discussed local performance sensitivity to elevation mask back in section 5.2.1. Now we look at the global trends, starting with results for GNSS only in Figure 5.9. The availability value is represented by the colors that go from red ( $0 \%$ availability) to blue ( $100 \%$ availability). For this value of HAL, the GNSS only case provides $0 \%$ availability for both scenarios.
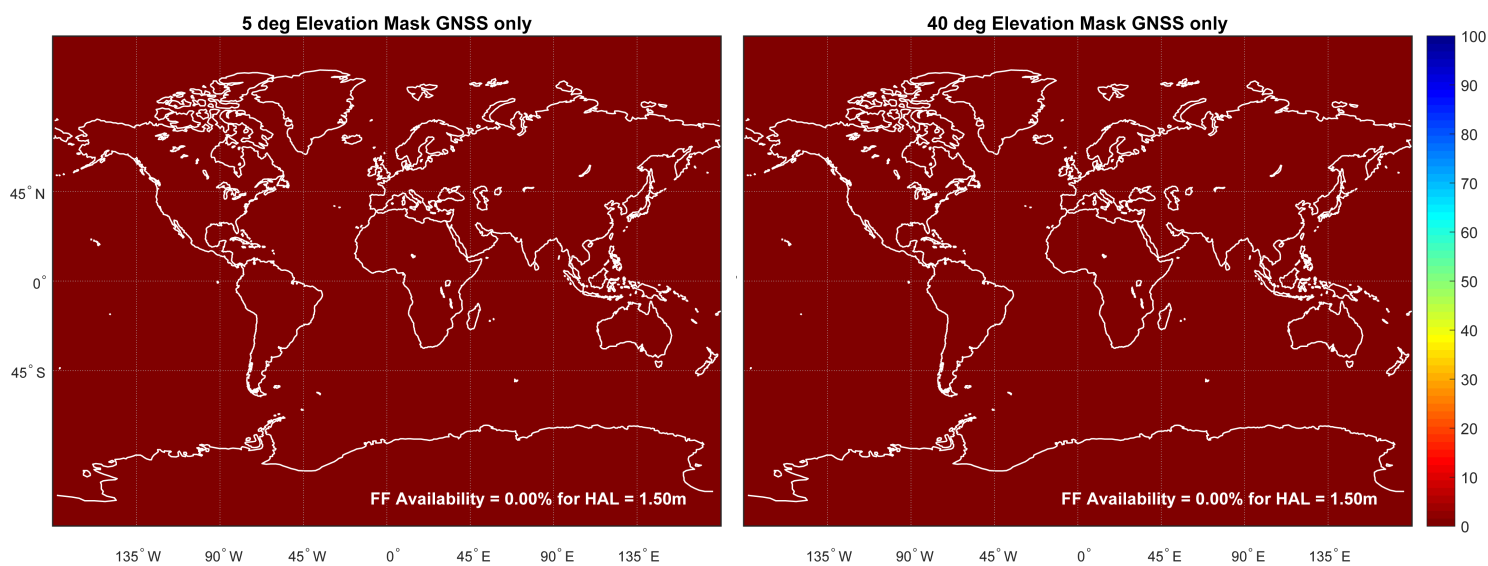

Figure 5.9: Fault-Free Availability Maps for 1.5m HAL using GNSS only. The availability maps show the horizontal positioning performance using GNSS only for open sky (left plot) and urban canyon (right plot) scenarios. The longitude and latitude is indicated in degree values in the $\mathrm{x}$-axis and $\mathrm{y}$-axis respectively. Each grid point is assigned a color that represents the availability value at that location. The color bar on the right indicates the availability value for each color.

The availability map of GNSS-OneWeb for the open sky scenario (left plot of Figure 5.10) shows 100\% fault-free availability from mid to high latitudes ( 45 to $90^{\circ} \mathrm{N}-\mathrm{S}$ ), and fault-free availability values in the 90 's range for the equatorial regions $\left(0\right.$ to $\left.45^{\circ} \mathrm{N}-\mathrm{S}\right)$ for a HAL value of $1.5 \mathrm{~m}$. This is caused by OneWeb's near polar orbits. Looking back to Figure 3.2, the satellites are denser at the poles and sparser in the equatorial region. For a 5-deg elevation mask, GNSS-OneWeb provides $96.96 \%$ fault-free availability for a horizontal alert limit of $1.5 \mathrm{~m}$ in the 24-hour simulation period. Note that this value is the result of weighting each availability value by cosine of latitude, which takes into account an ellipsoidal Earth where higher latitudes constitutes a smaller area of the map. 


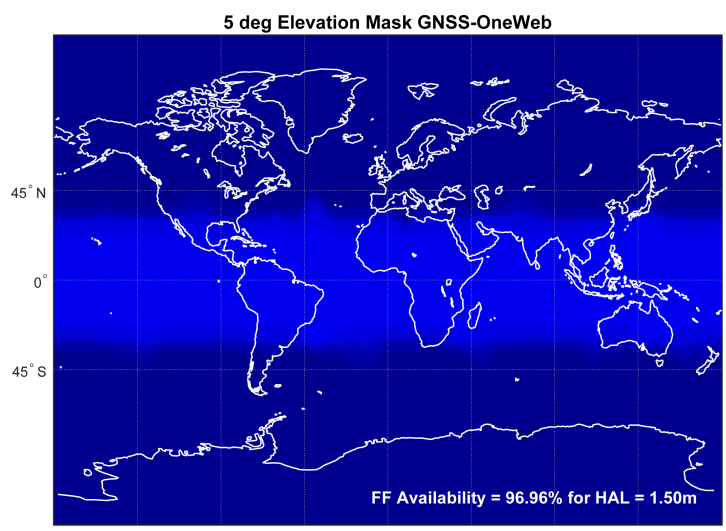

$135^{\circ} \mathrm{W} \quad 90^{\circ} \mathrm{W} \quad 45^{\circ} \mathrm{W} \quad 0^{\circ} \quad 45^{\circ} \mathrm{E} \quad 90^{\circ} \mathrm{E} \quad 135^{\circ} \mathrm{E}$

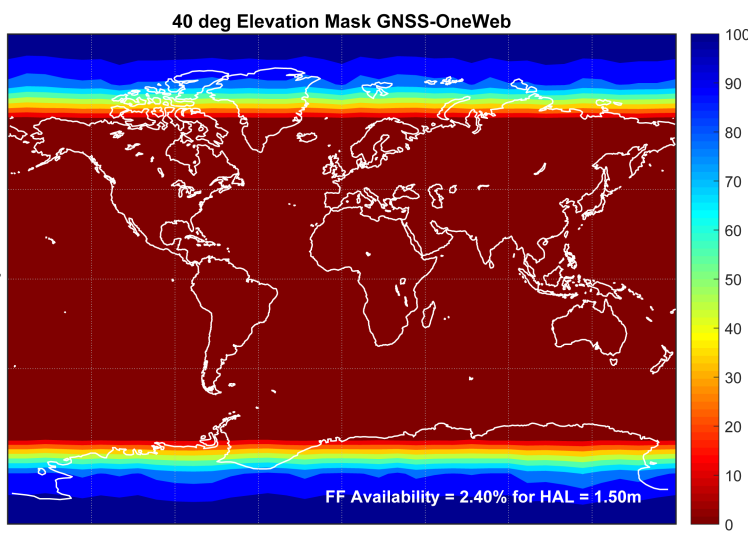

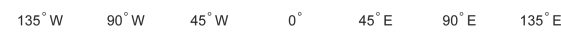

Figure 5.10: [Fault-Free Availability Maps for 1.5m HAL using GNSS-OneWeb. The availability maps show the horizontal positioning performance using GNSS-OneWeb for open sky (left plot) and urban canyon (right plot) scenarios. The longitude and latitude is indicated in degree values in the $\mathrm{x}$-axis and $y$-axis respectively. Each grid point is assigned a color that represents the availability value at that location. The color bar on the right indicates the availability value for each color.

The plot on the right of Figure 5.10 shows the availability map using GNSS-OneWeb for a 40-deg elevation mask. This shows that increasing the elevation mask from $5^{\circ}$ to $40^{\circ}$ has decreased the FF availability using GNSS-OneWeb from $96.96 \%$ to $2.40 \%$. Both scenarios show an improvement over using GNSS alone.

The open sky availability map using GNSS-Boeing (plot on the left of Figure 5.11) is a solid blue, indicating $100 \%$ fault-free availability worldwide for a horizontal alert limit of 1.5 meters in the 24-hour simulation period.

The urban canyon availability map on the right of Figure 5.11 shows $85.63 \%$ fault-free availability worldwide for an elevation mask of 40 degrees, where we see different performances for three distinct regions. There are two mechanisms at work here. The first involves the fraction of Boeing satellites inclined at $45^{\circ}$ and $55^{\circ}$. From basic orbital mechanics, we know that the geographic latitudes covered by a satellite's ground track ranges between \pm the inclination. The second mechanism concerns the fraction of Boeing satellites in near polar orbits, which will have a distribution that is denser at the 
poles, and sparser at the equatorial regions. We see both of these mechanisms at work in Figure 5.11, where there are red regions around $70^{\circ} \mathrm{N}-\mathrm{S}$, dark blue regions at low to mid-latitudes $\left(20^{\circ}-45^{\circ}\right)$, and lighter blues in the equatorial region. This plot shows that $100 \% \mathrm{FF}$ availability in the continental US is achievable for a HAL value of $1.5 \mathrm{~m}$. Both open sky and urban canyon availability maps show significant improvement over using GNSS only and GNSS-OneWeb.

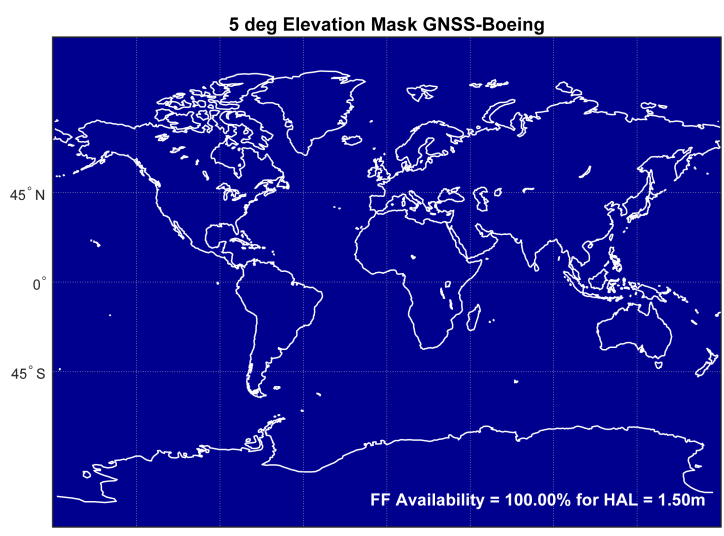

$135^{\circ} \mathrm{W} \quad 90^{\circ} \mathrm{W} \quad 45^{\circ} \mathrm{W} \quad 0^{\circ} \quad 45^{\circ} \mathrm{E} \quad 90^{\circ} \mathrm{E} \quad 135^{\circ} \mathrm{E}$

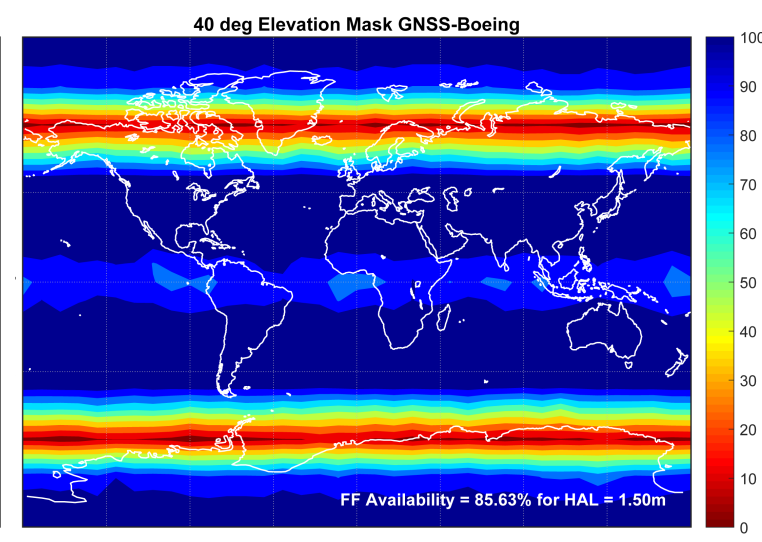

$\begin{array}{lllllll}135^{\circ} \mathrm{W} & 90^{\circ} \mathrm{W} & 45^{\circ} \mathrm{W} & 0^{\circ} & 45^{\circ} \mathrm{E} & 90^{\circ} \mathrm{E} & 135^{\circ} \mathrm{E}\end{array}$

Figure 5.11: [Fault-Free Availability Maps for 1.5m HAL using GNSS-Boeing. The availability maps show the horizontal positioning performance using GNSS-Boeing for open sky (left plot) and urban canyon (right plot) scenarios. The longitude and latitude is indicated in degree values in the $\mathrm{x}$-axis and $\mathrm{y}$-axis respectively. Each grid point is assigned a color that represents the availability value at that location. The color bar on the right indicates the availability value for each color.

The table below shows the minimum values for HAL needed to achieve $100 \%$ worldwide fault-free availability using GNSS only, GNSS-OneWeb, and GNSS-Boeing, for both open sky (5-deg elevation mask), and urban canyon scenarios (40-deg elevation mask). This table shows clearly the reduction in required HAL GNSS-MC can provide as compared to GNSS only.

Table 5.1: Minimum HAL Value to Achieve 100\% Worldwide FF Availability

\begin{tabular}{cccc}
\hline & GNSS only & GNSS-OneWeb & GNSS-Boeing \\
\hline $5 \mathrm{deg}$ & $11.37 \mathrm{~m}$ & $1.69 \mathrm{~m}$ & $0.58 \mathrm{~m}$ \\
$40 \mathrm{deg}$ & $380,781.66 \mathrm{~m}$ & $76,618.94 \mathrm{~m}$ & $5.23 \mathrm{~m}$ \\
\hline
\end{tabular}




\subsubsection{Fault-Free Availability Analysis for Varying HAL}

The horizontal alert limit used in the global maps of the previous section was chosen as a starting point to emphasize global trends when using either augmentation. These results showed little variability in the availability values across different longitudes, and showed symmetry in the availability values along the equator. Based on this information, we can look at the average performance at different latitudes for varying values of HAL. These results are shown in the next figures. The purpose of these plots is to show what FF availability values can be achieved at different latitudes for a range of HAL values between $0.5 \mathrm{~m}$ and $2 \mathrm{~m}$, for open sky (5-deg elevation mask) and urban canyon (40-deg elevation mask) scenarios.
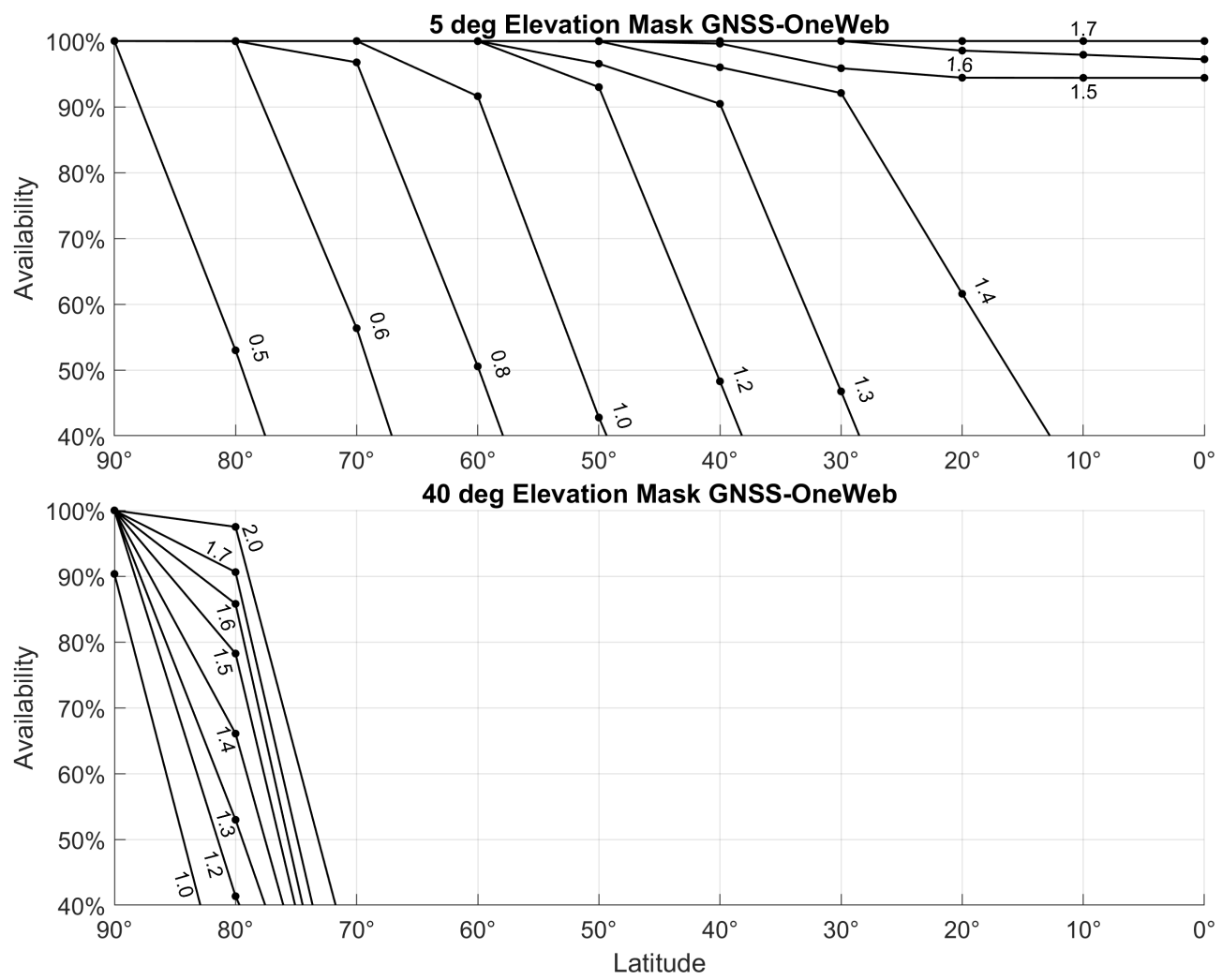

Figure 5.12: HAL Sensitivity using GNSS-OneWeb. These plots show the availability values at different latitudes for varying values of HAL, for a 5-deg elevation mask (top plot) and a 40-deg elevation mask (bottom plot), using OneWeb-augmented GNSS.

The top plot of Figure 5.12 shows the results using GNSS-OneWeb and a 5-deg elevation mask. Increasing the value of HAL up to $1.7 \mathrm{~m}$ ensures $100 \% \mathrm{FF}$ availability for all latitudes. For the continental 
US, which is at latitudes between $35-50^{\circ}$, HAL values as low as $1.4 \mathrm{~m}$ yield FF availability values greater than $90 \%$. When the elevation mask is increased to $40^{\circ}$ (bottom plot of Figure 5.12), FF availability values drop below $40 \%$ for latitudes lower than $80^{\circ}$ for the range of HAL values between $1.2 \mathrm{~m}$ and $2 \mathrm{~m}$.

The top plot of Figure 5.13 shows that for a 5 -deg elevation mask, 100\% FF availability can be achieved using GNSS-Boeing for a HAL as low as $0.6 \mathrm{~m}$. Even a lower HAL value of $0.5 \mathrm{~m}$ can still achieve $100 \%$ FF availability for the continental US (latitudes between $35-50^{\circ}$ ). When the elevation mask is increased to $40^{\circ}$ (bottom plot of Figure 5.13), we see poor performance towards the equator ( $0^{\circ}$ latitude), and the worst performance centered at $70^{\circ} \mathrm{N}$-S latitude. The cause for this result was explained for Figure 5.11 in the previous section. For the continental US, a HAL value of $1.4 \mathrm{~m}$ still achieves FF availability values greater than $90 \%$. This is a significant improvement over the GNSS-OneWeb and GNSS only case.

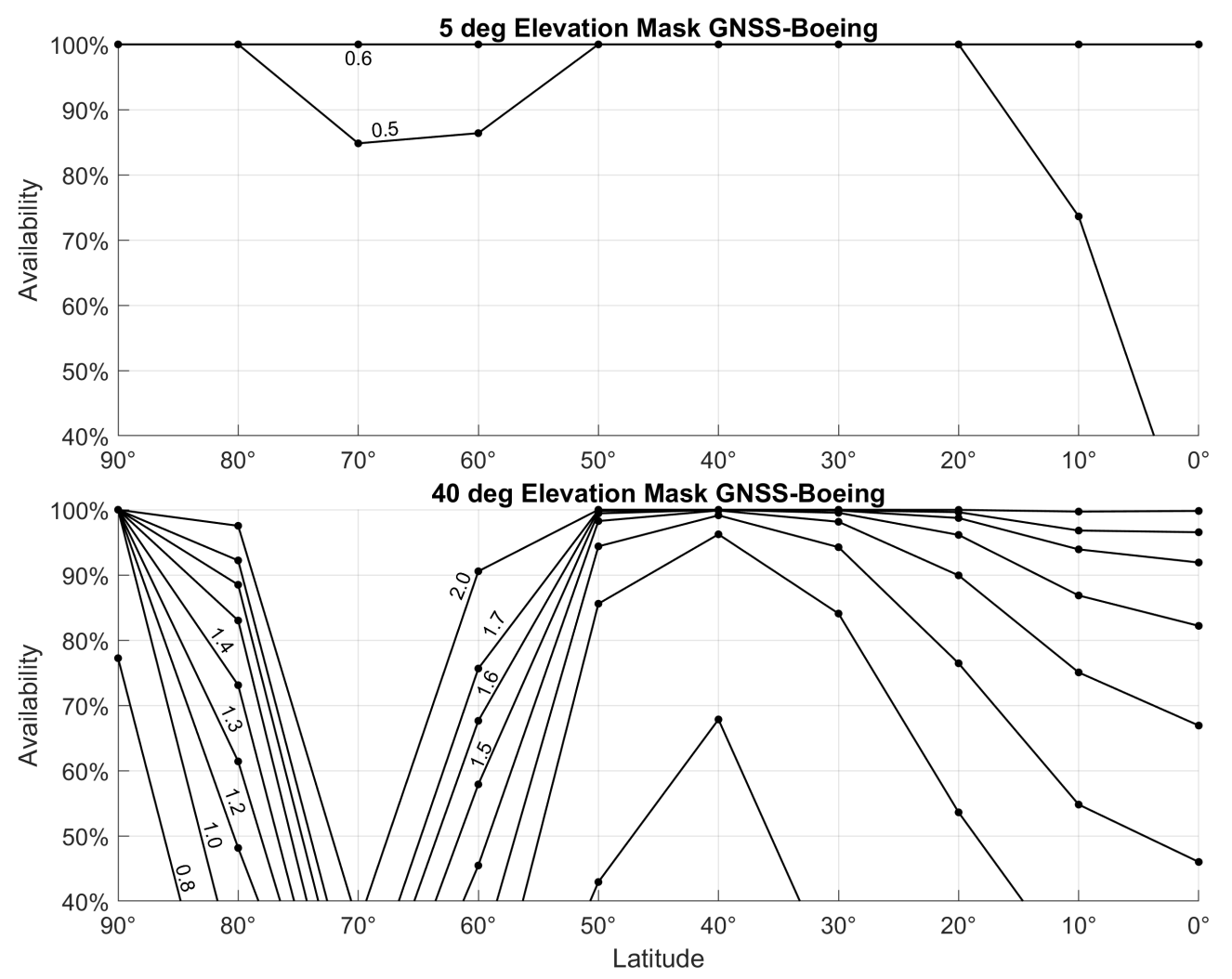

Figure 5.13: HAL Sensitivity using GNSS-Boeing. These plots show the availability values at different latitudes for varying values of HAL, for a 5-deg elevation mask (top plot) and a 40-deg elevation mask (bottom plot), using Boeing-augmented GNSS. 


\section{Chapter 6}

\section{Conclusions}

Carrier phase positioning requires estimating unknown carrier phase cycle ambiguities that remain constant, as long as the carrier is tracked continuously. This problem can be solved quickly provided that redundant satellites achieve substantial angular variations. With respect to users on Earth, the geometry of GNSS satellites at medium-Earth orbit (MEO) is slowly changing, which makes its use for carrier phase positioning impractical. On the other hand, soon to be deployed mega-constellations at low-Earth orbit (LEO) traverse wide sections of the sky within a few minutes. Signals from LEO satellites can provide the geometric diversity needed for rapid estimation of cycle ambiguities, and consequently, timely carrier phase positioning. Moreover, the considerable increase in ranging measurements provided by mega-constellations can provide robustness in urban canyon environments where a user has a narrow view of the sky, and low-elevation satellite signals are blocked.

\subsection{Summary of Contributions}

Derivation of Nominal Measurement Error Models for LEO Mega-Constellations. We developed new measurement error models for dual-frequency carrier phase measurements from LEO mega-constellations. Measurement error model parameters were defined for land vehicle applications. An algorithm was derived for integrating dual-frequency measurements from GNSS with dual-frequency carrier phase measurements from LEO mega-constellations, which (1) leveraged satellite motion to estimate cycle 
ambiguities and achieve rapid carrier phase positioning, and (2) accounted for time correlation in the errors which have not been addressed in prior models. The derived error models and batch measurement equations resulted in a compact positioning algorithm for mega-constellation-augmented GNSS (GNSS-MC).

Probabilistic Error Modeling using Two-Line Elements (TLEs). We quantified spacecraft positioning errors for 148,984 MEO TLE sets and 5813 geostationary Earth orbit (GEO) TLEs. TLE errors have significantly decreased over the past 20 years. In particular, after October 21, 2015, MEO GPS TLE errors become significantly smaller and more consistent as compared to their past history, whereas GEO WAAS TLE along-track and cross track errors tend to increase. In addition, we implemented overbounding theory to determine high-integrity GPS and GEO WAAS TLE error models. The same steps can be used in future work to validate other error models based on experimental data.

\section{Horizontal Positioning Error Sensitivity Analysis and Worldwide Fault-Free Availability Analysis. A} sensitivity analysis was implemented for a single reference location, to evaluate the effect of varying elevation mask, batch period, measurement sampling rate, and satellite ephemeris error, on the positioning performance using GNSS only, GNSS-OneWeb, and GNSS-Boeing. We showed that for high elevation masks ( $>40 \mathrm{deg}$ ), longer batch periods have a significant impact on positioning performance when using GNSS-only and GNSS-OneWeb, but not so much on GNSS-Boeing. We also showed that measurement sampling within the batch has a smaller impact, which is desired for computational efficiency, and which also indicates that changes in satellite geometry are key to reducing estimation errors, more so than noise averaging. The performance analysis culminated in a worldwide fault-free availability analysis which revealed global trends in the horizontal positioning performance using GNSS-OneWeb and GNSS-Boeing, and showed promise of reducing the required horizontal alert limit for GNSS-MC as compared to GNSS-only. 


\subsection{Recommended Topics for Future Work}

The weighting matrix used in the batch weighted least squares estimation algorithm is based on the error vector defined from the measurement error models. In this work, we used deterministic values for the error model parameters when in reality, these parameters have a range [48]. Accounting for ranges of parameter values can be achieved by incorporating Langel's proposed method of bounding integrity risk in batch estimation, where the weighting matrix has unknown, but bounded elements [50].

The positioning algorithm can be modified to incorporate ground application-specific means of performance improvement. More accurate satellite masks can be used i.e. use a cross shape for urban canyon intersections instead of a circle. Additional information such as an elevation (altitude) map can be incorporated to minimize the uncertainty in the vertical (down) position, and can allow positioning with one less ranging measurement. Also, knowledge of vehicle dynamics and measurement from an inertial navigation system (INS) can be considered in the estimation process.

There are ultimately many configurations that can make use of signals from LEO mega-constellations to augment GNSS. This work assumed one configuration where the LEO constellations had a dedicated ground control segment. Future work can consider the case where orbit and clock determination is done aboard the LEO spacecraft through a space-capable GNSS receiver. There are many advantages to this. The errors due to ionospheric and tropospheric delay is negligible. Precise orbit determination with onboard GNSS receivers were proven in [51] to be centimeter-level. Here however, we run into the problem of cascading error sources. Errors in GNSS clock and orbit ephemeris are inherited by the LEO spacecraft, which in turn gets passed down to the user on the ground.

Another topic for future work is evaluating the unprecedented potential of dual-frequency mega-constellation signals in sampling the ionosphere. With the addition of mega-constellations, we would have more pierce points in the ionosphere, and more sample total electron count (TEC) measurements with tighter resolution than ever before. 
Furthermore, the analysis needs to be refined to include fault detection. The impact of faults depend on the system architecture, e.g. cascading effect if mega-constellations use GPS for orbit and clock determination. 


\section{Bibliography}

[1] R. J. Danchik, "An overview of transit development," Johns Hopkins APL Technical Digest (Applied Physics Laboratory), vol. 19, no. 1, pp. 18-26, 1998. 11

[2] M. Rabinowitz, A Differential Carrier-Phase Navigation System Combining GPS with Low Earth Orbit Satellites for Rapid Resolution of Integer Cycle Ambiguities. PhD thesis, Stanford University, 2001. 11

[3] M. Joerger, J. Neale, B. Pervan, and S. Datta-Barua, "Measurement Error Models and Fault-Detection Algorithms for Multi-Constellation Navigation Systems," in IEEE/ION Position, Location and Navigation Symposium, pp. 927-946, 2010. 12

[4] C. T. Ardito, J. J. Morales, J. J. Khalife, A. A. Abdallah, and Z. M. Kassas, "Performance Evaluation of Navigation Using LEO Satellite Signals with Periodically Transmitted Satellite Positions," pp. $306-318,2016.12,42$

[5] T. G. Reid, A. M. Neish, T. Walter, and P. K. Enge, "Broadband LEO Constellations for Navigation," Navigation, Journal of the Institute of Navigation, vol. 65, no. 2, pp. 205-220, 2018. $12,30,38$

[6] J. Lee, Y. T. Morton, J. Lee, H. S. Moon, and J. Seo, "Monitoring and Mitigation of Ionospheric Anomalies for GNSS-Based Safety Critical Systems: A review of up-to-date signal processing techniques," IEEE Signal Processing Magazine, vol. 34, no. 5, pp. 96-110, 2017. 13

[7] M. Joerger, J. Neale, S. Datta-Barua, and B. Pervan, "Ionospheric error modeling for carrier phasebased multiconstellation navigation systems," IEEE Transactions on Aerospace and Electronic Systems, vol. 49, no. 1, pp. 451-467, 2013. 13

[8] Radio Technical Commission for Aeronautics (RTCA) Special Committee 159, "Minimum Operational Performance Standards for Global Positioning System/Wide Area Augmentation System Airborne Equipment," 2009. 13, 37, 38, 39, 40, 41, 57

[9] D. Racelis and M. Joerger, "High-Integrity TLE Error Models for MEO and GEO Satellites," in 2018 AIAA SPACE and Astronautics Forum and Exposition, 2018. 14, 33, 42

[10] P. Misra and P. Enge, Global Positioning System: Signals, Measurements, and Performance. Lincoln, Massachusetts: Ganga-Jamuna Press, revised se ed., 2012. 16, 17, 19, 20, 21, 23, 24, 25, 26, 27 , $28,29,49,50$ 
[11] B. W. Parkinson and J. J. Spilker, "Global positioning system: Theory and applications, volume 1," AIAA Progress in Aeronautics and Astronautics, vol. 163, 1996. 19

[12] P. Misra, GPS for Everyone: You are Here. Lincoln, Massachusetts: Ganga-Jamuna Press, second printing revised and updated ed., 2018. 19

[13] National Coordination Office for Space-Based Positioning, Navigation, and Timing, "GPS.gov: New Civil Signals.” https://www.gps.gov/systems/gps/modernization/civilsignals/, June 2016. (Accessed on 07/05/2019). 20

[14] U. USA: Department Of Defense, "Global Positioning System Standard Positioning Service," Www.Gps.Gov, no. September, pp. 1 - 160, 2008. 20

[15] Radio Technical Commission for Aeronautics (RTCA) Special Committee 159, "Minimum Aviation System Performance Standards for the Local Area Augmentation System (LAAS),” 2004. $24,25,31,43$

[16] G. Vijayaraghavan, M. Brown, and M. Barnes, "Electrical Noise and Mitigation - Part 1: Noise Definition, Categories and Measurement." https://www. eetimes.com/document.asp?doc_id= 1274125\#, December 2008. (Accessed on 07/06/2019). 25

[17] ESA, "Galileo Begins Serving the Globe." http://m.esa.int/Our_Activities/Navigation/ Galileo_begins_serving_the_globe. (Accessed on 07/06/2019). 26

[18] ESA, “Galileo Satellites." https://m.esa.int/Our_Activities/Navigation/Galileo/ Galileo_satellites. (Accessed on 07/06/2019). 26

[19] S. P. Diaz, M. Meurer, M. Rippl, and B. Belabbas, "URA/SISA Analysis for GPS-Galileo ARAIM Integrity Support Message," Proceedings of the 28th International Technical Meeting of The Satellite Division of the Institute of Navigation (ION GNSS+2015), pp. 735-745, 2015. 26, 43

[20] S. Perea, M. Meurer, llaria Martini, M. Rippl, M. Joerger, and B. Pervan, "Nominal Range Error Analysis to Support ARAIM," Proceedings of the 29th International Technical Meeting of The Satellite Division of the Institute of Navigation (ION GNSS+2016), pp. 1726-1735, 2018. 26

[21] S. Perea, M. Meurer, and B. Pervan, "Impact of Sample Correlation on SISRE Overbound for ARAIM," Proceedings of the 31 st International Technical Meeting of The Satellite Division of the Institute of Navigation (ION GNSS+2018), pp. 2567-2582, 2018. 26

[22] P. Y. Hwang, "Kinematic GPS for Differential Positioning: Resolving Integer Ambiguities on the Fly,” Navigation, vol. 38, no. 1, pp. 1-15, 1991. 28

[23] R. Kelly and J. Davis, "Required Navigation Performance (RNP) for Precision Approach and Landing with GNSS Application," Navigation, vol. 41, no. 1, 1994. 31

[24] J. Rife, S. Pullen, P. Enge, and B. Pervan, "Paired Overbounding for Nonideal LAAS and WAAS," IEEE Transactions on Aerospace and Electronic Systems, vol. 42, no. 4, pp. 1386-1395, 2006. 33, 42,43 
[25] B. DeCleene, “Defining Pseudorange Integrity - Overbounding," International Technical Meeting of the Satellite Division of The Institute of Navigation (ION GPS 2000), no. September, pp. 1916 1924, 2000. 33, 42, 43

[26] C. Henry, "OneWeb Shifts First Launch to Year's End." https://spacenews.com/ oneweb-shifts-first-launch-to-years-end/, May 2018. (Accessed on 06/27/2019). 34

[27] R. J. Barnett, "OneWeb Non-Geostationary Satellite System: Technical Information to Supplement Schedule S,” 2016. 34, 35, 36

[28] A. Nyirady, "OneWeb Successfully Launches 1st Batch of Satellites.” https://www. satellitetoday.com/launch/2019/02/28/ oneweb-successfully-launches-1st-batch-of-satellites/, February 2019. (Accessed on 06/27/2019). 34

[29] C. Henry, "Boeing Constellation Stalled, SpaceX Constellation Progressing." https: //spacenews.com/boeing-constellation-stalled-spacex-constellation-progressing/, June 2018. (Accessed on 06/27/2019). 35

[30] P. B. de Selding, "Boeing Proposes Big Satellite Constellations in V- and C-bands.” https:// spacenews .com/boeing-proposes-big-satellite-constellations-in-v-and-c-bands/, June 2016. (Accessed on 06/27/2019). 35

[31] W. A. Hanson, "In Their Own Words, OneWeb Internet Constellation as Described in their FCC From 312 Application,” 2016. 35, 36

[32] T. Azzarelli, "OneWeb: Access for Everyone," in ITU International Satellite Symposium, 2016. 36

[33] Working Group C, "EU-US Cooperation on Satellite Navigation Working Group C, ARAIM Technical Subgroup, Milestone 3 Report,” no. 1, pp. 1-76, 2016. 38, 41, 43

[34] Radio Technical Commission for Aeronautics (RTCA) Special Committee 159, "Minimum Operational Performance Standards for Global Positioning System/Wide Area Augmentation System Airborne Equipment," 2006. 39

[35] S. Khanafseh, B. Kujur, M. Joerger, T. Walter, S. Pullen, J. Blanch, K. Doherty, L. Norman, L. de Groot, and B. Pervan, "GNSS Multipath Error Modeling for Automotive Applications," Proceedings of the 31st International Technical Meeting of The Satellite Division of the Institute of Navigation (ION GNSS+2018), pp. 1573-1589, 2018. 40

[36] M. Joerger, Carrier Phase GPS Augmentation Using Laser Scanners and Using Low Earth Orbiting Satellites. PhD thesis, Illinois Institute of Technology, 2009. 40, 61

[37] T. Walter, K. Gunning, R. Eric Phelts, and J. Blanch, "Validation of the Unfaulted Error Bounds for ARAIM," Navigation: Journal of The Institute of Navigation, vol. 65, no. 1, pp. $117-133,2018$. 42,43 
[38] C. Cohenour and F. van Graas, "GPS Orbit and Clock Error Distributions," Navigation, vol. 58, no. 1, pp. 17-28, 2011. 42, 43

[39] Working Group C, "EU-US Cooperation on Satellite Navigation Working Group C, ARAIM Technical Subgroup, Milestone 2 Report," pp. 1-76, 2015. 43

[40] T. S. Kelso, "Validation of SGP4 and IS-GPS-200D Against GPS Precision Ephemerides," in 17th AAS/AIAA Space Flight Mechanics Conference, (Sedona, Arizona), AAS, 2007. 43

[41] D. A. Vallado and P. J. Cefola, "Two-Line Element Sets - Practice and Use," in 63rd International Astronautical Congress, (Naples, Italy), IAF, 2012. 43

[42] K. Riesing, "Orbit Determination from Two Line Element Sets of ISS-Deployed CubeSats," 29th Annual AIAA/USU Conference on Small Satellites. Student Competition, 2015. 43

[43] T. Flohrer, H. Krag, H. Klinkrad, B. B. Virgili, and C. Früh, "Improving ESA's Collision Risk Estimates by an Assessment of the TLE Orbit Errors of the US SSN Catalogue," in 5th European Conference on Space Debris, (Darmstadt, Germany), 2009. 43

[44] M. Joerger and B. Pervan, "Multi - Constellation ARAIM Exploiting Satellite Motion," 49

[45] M. Joerger and B. Pervan, "Exploiting Satellite Motion in ARAIM: Measurement Error Model Refinement Using Experimental Data," in Proceedings of the 29th International Technical Meeting of The Satellite Division of the Institute of Navigation (ION GNSS + 2016), 2016. 49

[46] M. Joerger, S. Stevanovic, F.-C. Chan, S. Langel, and B. Pervan, "Integrity Risk and Continuity Risk for Fault Detection and Exclusion Using Solution Separation ARAIM," in Proceedings of the 26th International Technical Meeting of The Satellite Division of the Institute of Navigation (ION GNSS 2013), 2013. 49

[47] M. Joerger, J. Neale, and B. Pervan, "Iridium/GPS carrier phase positioning and fault detection over wide areas," 22nd International Technical Meeting of the Satellite Division of the Institute of Navigation 2009, ION GNSS 2009 Volume 3, 2009, Pages 1685-1699, vol. 3, no. November, pp. $1685-1699,2009.49$

[48] B. Pervan, Navigation Integrity for Aircraft Precision Landing Using the GPS. PhD thesis, Stanford University, 1996. 51, 79

[49] S. Tongleamnak and M. Nagai, "Simulation of GNSS Availability in Urban Environments Using a Panoramic Image Dataset," International Journal of Navigation and Observation, vol. 2017, pp. 1 $12,2017.62$

[50] S. E. Langel, Bounding Estimation Integrity Risk for Linear Systems with Structured Stochastic Modeling Uncertainty. PhD thesis, Illinois Institute of Technology, 2014. 79

[51] Z. Kang, B. Tapley, S. Bettadpur, J. Ries, and P. Nagel, "Precise orbit determination for GRACE using accelerometer data," Advances in Space Research, vol. 38, no. 9, pp. 2131-2136, 2006. 79 